\title{
Bounded Model Checking of an MITL Fragment for Timed Automata
}

\author{
Roland Kindermann, Tommi Junttila and Ilkka Niemelä \\ Department of Information and Computer Science \\ Aalto University \\ P.O.Box 15400, FI-00076 Aalto, Finland \\ Email: \{Roland.Kindermann,Tommi.Junttila,Ilkka.Niemela $\} @$ aalto.fi
}

\begin{abstract}
Timed automata (TAs) are a common formalism for modeling timed systems. Bounded model checking (BMC) is a verification method that searches for runs violating a property using a SAT or SMT solver. MITL is a real-time extension of the linear time logic LTL. Originally, MITL was defined for traces of non-overlapping time intervals rather than the "super-dense" time traces allowing for intervals overlapping in single points that are employed by the nowadays common semantics of timed automata. In this paper we extend the semantics of a fragment of MITL to super-dense time traces and devise a bounded model checking encoding for the fragment. We prove correctness and completeness in the sense that using a sufficiently large bound a counter-example to any given non-holding property can be found. We have implemented the proposed bounded model checking approach and experimentally studied the efficiency and scalability of the implementation.
\end{abstract}

Keywords-timed automaton; metric interval temporal logic; bounded model checking; satisfiability modulo theories

\section{INTRODUCTION}

Fully-automated verification has many industrial applications. A particularly interesting and challenging setting for the use of verification are systems for which timing aspects are of high importance like safety instrumented systems or communication protocols. In this paper, we study verification in a setting where both the system and the specification contain quantitative timing aspects, allowing not only to specify, e.g., that a certain situation will eventually lead to a reaction but also that the reaction will happen within a certain amount of time. Allowing such timing aspects to be part of both the specification and the system adds an additional challenge.

Timed automata [1] are a widely employed formalism for the representation of finite state systems augmented with real-valued clocks. Timed automata have been studied for two decades and various tools for the verification of timed automata exist. Most existing verification techniques and tools, like the model checker Uppaal [2], however do not support quantitative specifications on the timing of events. We feel that the ability to state, e.g., that a certain condition triggers a reaction within a certain amount of time provides a clear improvement over being able only to specify that a reaction will eventually occur. For specifications, we use the linear time logic $\mathrm{MITL}_{0, \infty}$ [3], an extension adding lower and upper time bounds to the popular logic LTL.

Industrial size systems often have a huge discrete state space in addition to the infinite state space of timingrelated parts of the system. We feel that fully symbolic verification is a key to tackling large discrete state spaces. We, thus, provide a translation of a pair of a timed automaton representing a system and a $\mathrm{MITL}_{0, \infty}$ formula into a symbolic transition system that can serve as a foundation for various symbolic verification methods. It is proven that the translated system has a trace if and only if the original timed automaton has a trace satisfying the formula. We, furthermore, demonstrate how to employ the translation for SMT-based bounded model checking using the regionabstraction for timed automata [1]. We show completeness of the approach and prove the applicability of the region abstraction to the transition system. Finally, we evaluate the scalability of the approach and the cost for checking specifications containing timing experimentally.

MITL $_{0, \infty}$ is a fragment of the logic MITL [3] for which the question whether or not a given timed automaton has a trace satisfying or violating a given formula is PSPACE complete [3]. Previously, a verification approach for $\mathrm{MITL}_{0, \infty}$ specifications was introduced in [3] and improved upon in [4]. At this point, however, there are to our best knowledge no implementations or results of experiments using these methods available. Additionally, a major difference between the techniques described in [3], [4] and our approach lies in the precise semantics of timed automata used. While previous approaches use dense-time semantics, we extend MITL $_{0, \infty}$ to super-dense time. Although dense and superdense time semantics of timed automata are often used interchangeably in the literature (and in fact do not differ in any important fashion when, e.g., verifying reachability constraints), we will show that equivalences between $\mathrm{MITL}_{0, \infty}$ formulas fundamental to the techniques in [3], [4] do not hold anymore when using dense-time semantics.

\section{Timed AutomatA}

We first give basic definitions for timed automata (see e.g. [1], [5], [6]). For simplicity, we use basic timed automata in the theoretical parts of the paper. However, in practice (and the experimental part of the paper) one usually defines a network of timed automata that can also have (shared and 


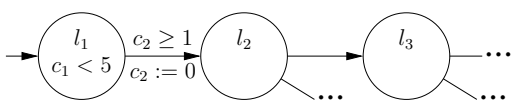

Figure 1. A timed automaton

local) finite domain non-clock variables manipulated on the edges. The symbolic bounded model checking encodings presented later in the paper can be extended to handle both of these features: see, e.g., [7], [8] on how to handle synchronization in a network of timed automata. Alternatively, one can specify timed systems with a symbolic formalism [9].

Let $X$ be a set of real-valued clock variables. A clock valuation $\nu$ is a function $\nu: X \rightarrow \mathbb{R}_{\geq 0}$. For $\delta \in \mathbb{R}_{\geq 0}$ we define the valuation $\nu+\delta$ by $\forall x \in X:(\nu+\delta)(x)=\nu(x)+\delta$. The set of clock constraints over $X, \mathcal{C}(X)$, is defined by the grammar $C::=$ true $|x \bowtie n| C \wedge C$ where $x \in X$, $\bowtie \in\{<, \leq,=, \geq,>\}$ and $n \in \mathbb{N}$. A valuation $\nu$ satisfies $C \in \mathcal{C}(X)$, denoted by $\nu \models C$, if it evaluates $C$ to true.

A timed automaton (TA) is a tuple $\left\langle L, l_{\text {init }}, X, E, I\right\rangle$ where

- $L$ is a finite set of locations,

- $l_{\text {init }} \in L$ is the initial location of the automaton,

- $X$ is a finite set of real-valued clock variables,

- $E \subseteq L \times \mathcal{C}(X) \times 2^{X} \times L$ is a finite set of edges, each edge $\left\langle l, g, R, l^{\prime}\right\rangle \in E$ specifying a guard $g$ and a set $R$ of clocks to be reset, and

- $I: L \rightarrow \mathcal{C}(X)$ assigns an invariant to each location.

As an example, Figure 1 shows a part of a timed automaton with locations $l_{1}, l_{2}, \ldots$, and two clocks $c_{1}$ and $c_{2}$. The initial location is $l_{1}$, having the invariant $c_{1}<5$. The invariant of the location $l_{2}$ is true. The edge from $l_{1}$ to $l_{2}$ has the guard $c_{2} \geq 1$ and the reset set $\left\{c_{2}\right\}$. The guard of the edge from $l_{2}$ to $l_{3}$ is true and its reset set is empty.

A state of a timed automaton $\mathcal{A}=\left\langle L, l_{\text {init }}, X, E, I\right\rangle$ is a pair $\langle l, \nu\rangle$, where $l \in L$ is a location and $\nu$ is a clock valuation over $X$. A state $\langle l, \nu\rangle$ is (i) initial if $l=l_{\text {init }}$ and $\nu(x)=0$ for each $x \in X$, and (ii) valid if $\nu \models I(l)$. Let $\langle l, \nu\rangle$ and $\left\langle l^{\prime}, \nu^{\prime}\right\rangle$ be states of $\mathcal{A}$. There is a time elapse step of $\delta \in \mathbb{R}_{>0}$ time units from $\langle l, \nu\rangle$ to $\left\langle l^{\prime}, \nu^{\prime}\right\rangle$, denoted by $\langle l, \nu\rangle \stackrel{\delta}{\rightarrow}\left\langle l^{\prime}, \nu^{\prime}\right\rangle$, if (i) $l=l^{\prime}$, (ii) $\nu^{\prime}=\nu+\delta$, and (iii) $\left\langle l^{\prime}, \nu^{\prime}\right\rangle$ is a valid state. Intuitively, there is a time elapse step from a state to another if the second state can be reached from the first one by letting $\delta$ amount of time pass. There is a discrete step from $\langle l, \nu\rangle$ to $\left\langle l^{\prime}, \nu^{\prime}\right\rangle$, denoted by $\langle l, \nu\rangle \stackrel{0}{\rightarrow}$ $\left\langle l^{\prime}, \nu^{\prime}\right\rangle$, if there is an edge $\left\langle l, g, R, l^{\prime}\right\rangle \in E$ such that (i) $\nu \models$ $g$, (ii) $\left\langle l^{\prime}, \nu^{\prime}\right\rangle$ is a valid state, and (iii) $\nu^{\prime}(x)=0$ for all $x \in R$ and $\nu^{\prime}(x)=\nu(x)$ for all $x \in X \backslash R$. That is, discrete steps can be used to change the current location as long as the guard and the target location invariant are satisfied. A discrete step resets some clocks and leaves the other's values unchanged, i.e., a discrete step does not take any time.

A run of $\mathcal{A}$ is an infinite sequence of states $\pi=$ $\left\langle l_{0}, \nu_{0}\right\rangle \stackrel{\delta_{0}}{\longrightarrow}\left\langle l_{1}, \nu_{1}\right\rangle \stackrel{\delta_{1}}{\longrightarrow} \ldots$, such that (i) $\left\langle l_{0}, \nu_{0}\right\rangle$ is valid and initial, and (ii) $\left\langle l_{i}, \nu_{i}\right\rangle \stackrel{\delta_{i}}{\longrightarrow}\left\langle l_{i+1}, \nu_{i+1}\right\rangle$ with some $\delta_{i} \in \mathbb{R}$ for each consecutive pair of states. E.g., the automaton in Figure 1 has a run $\left\langle l_{1},(0,0)\right\rangle \stackrel{3.5}{\rightarrow}\left\langle l_{1},(3.5,3.5)\right\rangle \stackrel{0}{\rightarrow}$ $\left\langle l_{2},(3.5,0.0)\right\rangle \stackrel{0}{\rightarrow}\left\langle l_{3},(3.5,0.0)\right\rangle \stackrel{1.1}{\rightarrow}\left\langle l_{3},(4.6,1.1)\right\rangle \ldots$ where each clock valuation $\left\{c_{1} \mapsto v, c_{2} \mapsto w\right\}$ is abbreviated with $(v, w)$. A run is non-zeno if the total amount $\sum_{i=0}^{\infty} \delta_{i}$ of time passed in the run is infinite. In the rest of the paper, we will only consider non-zeno runs.

Observe that on timed automata runs, the automaton can visit multiple locations without time elapsing in between. For instance, at the time point 3.5 in the run given above, the automaton is after the first time elapse step in location $l_{1}$, then after the first discrete step in location $l_{2}$, and finally after the second discrete step in location $l_{3}$. These kind of "super-dense" runs differ from the dense runs that can be represented with "signals", i.e. by mapping each time point in $\mathbb{R}_{\geq 0}$ to a single value. As we will see in the next section, considering super-dense timed automata runs complicates model checking as, e.g., we cannot get rid of the timed until operator in the way we would if dense runs were used.

Note that previous papers on timed automata use both dense (e.g. [1]) and super-dense time (e.g. [5]), often without addressing the different semantics. From a practical perspective, super-dense runs appear paradox, as they permit multiple successive events to happen with no time passing in between. An alternative way of interpreting super-dense time, however, is that the amount of time in between events is just too small to be of interest and is, thus, abstracted away. We also take the fact that Uppaal [2], arguably the most successful timed model checker, not only allows for super-dense time traces but actually even makes it possible to enforce super-dense behaviors by marking locations as "urgent" or "committed" as a strong indication that there is an interest in super-dense traces in practice.

\section{THE LOGIC MITL $0, \infty$ FOR SUPER-DENSE TIME}

Next, we describe the syntax and semantics of $\mathrm{MITL}_{0, \infty}$ formulas over "super-dense timed traces" which, as discussed in Sect. III-C, can represent timed automata runs.

\section{A. Syntax and Semantics}

Assuming a set $A P$ of atomic propositions, the syntax of $\mathrm{MITL}_{0, \infty}$ formula follows that in [3], and is defined by the BNF grammar $\phi::=p|\phi| \neg \phi|\phi \wedge \phi| \phi \vee \phi \mid$ $\phi \mathbf{U}_{\bowtie n}^{\mathrm{s}} \phi \mid \phi \mathbf{R}_{\bowtie n}^{\mathrm{s}} \phi$ where $p$ ranges over $A P, n$ ranges over $\mathbb{N}$, and $\bowtie$ ranges over $\{<, \leq, \geq,>\}$. Intuitively, a strict timed until formula $\phi \mathbf{U}_{\bowtie n}^{\mathrm{s}} \psi$ states that $\phi$ holds in all later time points until $\psi$ holds at a time point $t$ satisfying the timing constraint, i.e. $t \bowtie n$. Rational time constraints could be allowed in the temporal operators without influencing the expressivity of the logic (see [3] for MITL on dense traces). We define the usual abbreviations: true $\equiv(p \vee \neg p)$, false $\equiv$ $\neg$ true, $\mathbf{F}_{\bowtie n}^{\mathrm{s}} \phi \equiv$ true $\mathbf{U}_{\bowtie n}^{\mathrm{s}} \phi$, and $\mathbf{G}_{\bowtie n}^{\mathrm{s}} \phi \equiv$ false $\mathbf{R}_{\bowtie n}^{\mathrm{s}} \phi$. 
We now define the semantics of MITL $_{0, \infty}$ over "superdense" timed traces, and then later show the correspondence of timed automata runs to such traces. A super-dense timed trace over a set of atomic propositions $A P$ is an infinite sequence $\sigma=\left\langle I_{0}, v_{0}\right\rangle\left\langle I_{1}, v_{1}\right\rangle \ldots$, where

- each $v_{i}$ is a subset of $A P$,

- each $I_{i}$ is either an open interval $\left(T_{i}, T_{i}^{\prime}\right)$ or a singleton $\left[T_{i}, T_{i}\right]$ with $0 \leq T_{i}<T_{i}^{\prime}$ and $T_{i}, T_{i}^{\prime} \in \mathbb{R}_{\geq 0}$,

- $I_{0}=[0,0]$,

- for each $i \in \mathbb{N}$ it holds that (i) $I_{i}=\left(T_{i}, T_{i}^{\prime}\right)$ implies $I_{i+1}=\left[T_{i}^{\prime}, T_{i}^{\prime}\right]$, and (ii) $I_{i}=\left[T_{i}, T_{i}\right]$ implies either $I_{i+1}=\left[T_{i}, T_{i}\right]$ or $I_{i+1}=\left(T_{i}, T_{i+1}^{\prime}\right)$; and

- every $t \in \mathbb{R}_{\geq 0}$ is contained in at least one $I_{i}$.

For each trace element $\left\langle I_{i}, v_{i}\right\rangle$, equivalently written as $\left\langle I_{i}\right\rangle$, the interpretation is that the atomic propositions in $v_{i}$ hold in all the time points in the interval $I_{i}$. As consecutive singletons are allowed, it is possible for an atomic proposition to change its value an arbitrary finite number of times at a given time point. This is required to capture timed automata traces containing two or more successive discrete steps and differentiates super-dense timed traces from dense ones. In the semantics part we could have allowed general intervals; however, our constructions depend on discriminating the end points of left/right-closed intervals and thus we use this normal form already here. A dense timed trace is a superdense timed trace with no consecutive singletons (i.e., every time point $t \in \mathbb{R}_{\geq 0}$ occurs in exactly one $I_{i}$ ).

The set of all points in a trace $\sigma$ is defined by $T(\sigma)=\left\{(i, t) \mid i \in \mathbb{N}, t \in I_{i}\right\}$. Two points, $(i, t),\left(i^{\prime}, t^{\prime}\right) \in$ $T(\sigma)$, are ordered with the "earlier" relation $\prec$ defined by $(i, t) \prec\left(i^{\prime}, t^{\prime}\right) \Leftrightarrow i<i^{\prime} \vee\left(i=i^{\prime} \wedge t<t^{\prime}\right)$ and the set of all points later than $(i, t)$ is defined by $T_{+}(\sigma,(i, t)):=$ $\left\{\left(i^{\prime}, t^{\prime}\right) \in T(\sigma) \mid(i, t) \prec\left(i^{\prime}, t^{\prime}\right)\right\}$.

Given a super-dense timed trace $\sigma$ over $A P$, a formula $\phi$ over $A P$, and a point $(i, t)$ in $\sigma$, we define the satisfies relation $\sigma^{(i, t)}=\phi$ iteratively as follows:

- $\sigma^{(i, t)}=p$ iff $p \in v_{i}$, where $p$ is an atomic proposition.

- $\sigma^{(i, t)}=\neg \phi$ iff $\sigma^{(i, t)}=\phi$ does not hold.

- $\sigma^{(i, t)}=(\phi \wedge \psi)$ iff $\sigma^{(i, t)}=\phi$ and $\sigma^{(i, t)}=\psi$.

- $\sigma^{(i, t)}=(\phi \vee \psi)$ iff $\sigma^{(i, t)}=\phi$ or $\sigma^{(i, t)} \models \psi$.

- $\sigma^{(i, t)}=\left(\begin{array}{lll}\phi & \mathbf{U}_{\triangle n}^{\mathrm{s}} & \psi\end{array}\right)$ iff $\exists\left(i^{\prime}, t^{\prime}\right) \in T_{+}(\sigma,(i, t))$ : $\left(t^{\prime}-t \bowtie n\right) \wedge\left(\sigma^{\left(i^{\prime}, t^{\prime}\right)}=\psi\right) \wedge\left(\forall\left(i^{\prime \prime}, t^{\prime \prime}\right) \in T_{+}(\sigma,(i, t))\right.$ : $\left.\left(i^{\prime \prime}, t^{\prime \prime}\right) \prec\left(i^{\prime}, t^{\prime}\right) \Rightarrow\left(\sigma^{\left(i^{\prime \prime}, t^{\prime \prime}\right)}=\phi\right)\right)$

- $\sigma^{(i, t)}=\left(\phi \mathbf{R}_{\bowtie n}^{\mathrm{s}} \psi\right)$ iff $\forall\left(i^{\prime}, t^{\prime}\right) \in T_{+}(\sigma,(i, t))$ : $\left(\left(t^{\prime}-t \bowtie n\right) \wedge \neg\left(\sigma^{\left(i^{\prime}, t^{\prime}\right)}=\psi \psi\right)\right) \Rightarrow\left(\exists\left(i^{\prime \prime}, t^{\prime \prime}\right) \in\right.$ $\left.T_{+}(\sigma,(i, t)):\left(i^{\prime \prime}, t^{\prime \prime}\right) \prec\left(i^{\prime}, t^{\prime}\right) \wedge\left(\sigma^{\left(i^{\prime \prime}, t^{\prime \prime}\right)} \models \phi\right)\right)$

For any formula $\phi$, we abbreviate $\sigma^{(0,0)} \models \phi$ with $\sigma \models \phi$.

Example 1: Consider the super-dense timed trace $\sigma=$ $\left\langle\begin{array}{c}{[0,0]} \\ \emptyset\end{array}\right\rangle\left\langle\begin{array}{c}(0,4) \\ \{p\}\end{array}\right\rangle\left\langle\begin{array}{c}{[4,4]} \\ \{p\}\end{array}\right\rangle\left\langle\begin{array}{c}{[4,4]} \\ \{q\}\end{array}\right\rangle\left\langle\begin{array}{c}{[4,4]} \\ \emptyset\end{array}\right\rangle$... Now $\sigma \models p \mathbf{U}_{\leq 4}^{\mathrm{s}} q$ as $\sigma^{(3,4)} \models q$ and $\sigma^{(i, t)} \models p$ for all $0<i<3$ and $0 \leq t \leq 4$. As an another example, $\sigma \models \mathbf{F}_{\leq 3}^{\mathrm{s}}\left(\left(\mathbf{G}_{\leq 1}^{\mathrm{s}} p\right) \wedge\left(\mathbf{F}_{<2}^{\mathrm{s}} q\right)\right)$ also holds because (i) $\sigma^{(1, t)} \models \mathbf{G}_{\leq 1}^{\mathrm{s}} p$ for all $0 \leq t<3$, and (ii) $\sigma^{(1, t)} \mid=\mathbf{F}_{<2}^{\mathrm{s}} q$ for all $2<t<4$.
As illustrated in Ex. 1, neither $\phi$ nor $\psi$ need to hold in the current point in order to satisfy $\phi \mathbf{U}_{\bowtie n}^{\mathrm{s}} \psi$. Conversely, $\phi \mathbf{U}_{\triangleleft n}^{\mathrm{s}} \psi$ with $\triangleleft \in\{<, \leq\}$ does not necessarily hold even if $\psi$ holds in the first state: e.g., $\left\langle\begin{array}{c}{[0,0]} \\ \{q\}\end{array}\right\rangle\left\langle\begin{array}{c}(0,3) \\ \emptyset\end{array}\right\rangle$... does not satisfy $p \mathbf{U}_{<2}^{\mathrm{s}} q$. As [3] observes, the reason for this slightly unintuitive semantics is that they allow expressing formulas that would not be expressible if more intuitive semantics where the current point in time is relevant for the timed until operator as well were used. On the other hand, expressing that $\phi$ holds from the current point in time on until $\psi$ holds can be done using the formula $\psi \vee\left(\phi \wedge\left(\phi \mathbf{U}_{\bowtie n}^{\mathrm{s}} \psi\right)\right)$.

We can define the "untimed versions" of the temporal operators with $\mathbf{F}^{\mathrm{s}} \phi \equiv \mathbf{F}_{\geq 0}^{\mathrm{s}} \phi, \mathbf{G}^{\mathrm{s}} \phi \equiv \mathbf{G}_{\geq 0}^{\mathrm{s}} \phi, \phi \mathbf{U}^{\mathrm{s}} \psi \equiv$ $\phi \mathbf{U}_{>0}^{\mathrm{s}} \psi$, and $\phi \mathbf{R}^{\mathrm{s}} \psi \equiv \phi \mathbf{R}_{>0}^{\mathrm{s}} \psi$. An easily made misconception is that the time-aspect of a timed trace is irrelevant when evaluating "untimed" operators, i.e., that they could be evaluated on $\omega$-words obtained when removing intervals from a trace; this is not the case. In fact, even when not taking the "only in the future" part of the semantics, illustrated in the previous example, into account, considering the sets of propositions only is not sufficient. As an example, the formula $p \mathbf{U}^{\mathrm{s}} q$ is satisfied on $\left\langle\begin{array}{c}{[0,0]} \\ \{p\}\end{array}\right\rangle\left\langle\begin{array}{c}(0,2) \\ \{p\}\end{array}\right\rangle\left\langle\begin{array}{c}{[2,2]} \\ \{q\}\end{array}\right\rangle \ldots$ but not on $\left\langle\begin{array}{c}{[0,0]} \\ \{p\}\end{array}\right\rangle\left\langle\begin{array}{c}(0,2) \\ \{p\}\end{array}\right\rangle\left\langle\begin{array}{c}{[2,2]} \\ \{p\}\end{array}\right\rangle\left\langle\begin{array}{c}(2,3.5) \\ \{q\}\end{array}\right\rangle \ldots$. The issue in the second trace is that as the interval on which $q$ holds is an open one, any point in it has a previous point at which only $q$, but not $p$, holds. This illustrates that even for the "untimed" versions of the operators, timing is relevant.

Observe that with super-dense timed traces we cannot get rid of the timed until operator $\mathbf{U}_{\bowtie n}^{\mathrm{s}}$ by using the "timed until is redundant" theorem of [4], vital for the transducer construction presented there. That is, $\phi \mathbf{U}_{\geq n}^{\mathrm{s}} \psi$ is not equivalent to $\left(\mathbf{G}_{\leq n}^{\mathrm{s}}(\phi \mathbf{U} \psi)\right) \wedge \mathbf{F}_{\geq n}^{\mathrm{s}} \psi$ in our setting. ${ }^{1}$ For example, in the trace $\sigma=\left\langle\begin{array}{c}{[0,0]} \\ \{p\}\end{array}\right\rangle\left\langle\begin{array}{c}(0,2) \\ \{p\}\end{array}\right\rangle\left\langle\begin{array}{c}{[2,2]} \\ \{p\}\end{array}\right\rangle\left\langle\begin{array}{c}{[2,2]} \\ \{q\}\end{array}\right\rangle\left\langle\begin{array}{c}{[2,2]} \\ \emptyset\end{array}\right\rangle \ldots$ we have $\sigma \models p \mathbf{U}_{\geq 2}^{\mathrm{s}} q$ but $\sigma \not \models\left(\mathbf{G}_{\leq 2}^{\mathrm{s}}(p \mathbf{U} q)\right) \wedge \mathbf{F}_{\geq 2}^{\mathrm{s}} q$ as $\sigma^{(4,2)} \not \neq p \mathbf{U} q$. Likewise, the corresponding equivalences used in [3] do not hold when using super-dense time, e.g. $p \mathbf{U}_{>2}^{\mathrm{s}} q$ is not equivalent to $\mathbf{G}_{<2}^{\mathrm{s}} p \wedge \mathbf{G}_{\leq 2}^{\mathrm{s}}\left(q \vee\left(p \wedge\left(p \mathbf{U}^{\mathrm{s}} p\right)\right)\right)$ which can be demonstrated by the exact same trace.

Similarly, it is not possible to use the classic LTL equality $\phi \mathbf{R} \psi \equiv(\mathbf{G} \psi) \vee(\psi \mathbf{U}(\phi \wedge \psi))$ to handle timed release operator by means of the other operators in our setting: e.g., when $\sigma=\left\langle\begin{array}{c}{[0,0]} \\ \emptyset\end{array}\right\rangle\left\langle\begin{array}{c}(0,2) \\ \{\psi\}\end{array}\right\rangle\left\langle\begin{array}{c}{[2,2]} \\ \{\psi\}\end{array}\right\rangle\left\langle\begin{array}{c}(2,4) \\ \{\phi\}\end{array}\right\rangle \ldots$ we have $\sigma=$ $\phi \mathbf{R}_{\leq 3}^{\mathrm{s}} \psi$ but $\sigma \not \models \mathbf{G}_{\leq 3}^{\mathrm{s}} \psi$ and $\sigma \not \models \psi \mathbf{U}_{\leq 3}^{\mathrm{s}}(\phi \wedge \psi)$.

One can verify that the usual dualities hold for the operators: $\neg \neg \phi \equiv \phi, \neg(\phi \vee \psi) \equiv(\neg \phi) \wedge(\neg \psi), \neg(\phi \wedge$ $\psi) \equiv(\neg \phi) \vee(\neg \psi), \neg\left(\phi \mathbf{U}_{\bowtie n}^{\mathrm{s}} \psi\right) \equiv(\neg \phi) \mathbf{R}_{\bowtie n}^{\mathrm{s}}(\neg \psi)$, and $\neg\left(\phi \mathbf{R}_{\bowtie n}^{\mathrm{s}} \psi\right) \equiv(\neg \phi) \mathbf{U}_{\bowtie n}^{\mathrm{s}}(\neg \psi)$. These allow us to transform a formula into positive normal form in which negations only appear in front of atomic propositions. From now on, we assume that all formulas are in positive normal form.

\footnotetext{
${ }^{1}$ Here, $\mathbf{U}$ is the non-strict until operator, i.e. $\phi \mathbf{U} \psi:=\psi \vee\left(\phi \wedge\left(\phi \mathbf{U}^{\mathrm{s}} \psi\right)\right)$
} 


\section{B. Trace Refinement and Fineness}

To perform model checking of $\mathrm{MITL}_{0, \infty}$ formulas, we do not want the values of sub-formulas to change during open intervals. We next formalize this and show how it can be achieved by means of trace refinement; the definitions and results here are extended from those in Sect. 2 of [3].

A trace $\sigma^{\prime}$ is a refinement of a trace $\sigma$, denoted by $\sigma^{\prime} \preceq \sigma$, if it can be obtained by replacing each open interval $\left\langle\left(\begin{array}{c}\left(T_{i}, T_{i}^{\prime}\right) \\ v\end{array}\right\rangle\right.$ in the trace $\sigma$ with a sequence of intervals $\left\langle\begin{array}{c}\left(T_{i, 0}, T_{i, 1}\right) \\ v_{i}, v_{i}\end{array}\left\langle\left\langle T_{i, 1}, T_{i, 1}\right]\right\rangle\left\langle\left\langle\begin{array}{c}\left(T_{i, 1}, T_{i, 2}\right) \\ v_{i}\end{array} v_{i} \ldots\left\langle\left\langle\begin{array}{c}\left(T_{i, k-1}, T_{i, k}\right) \\ v_{i}\end{array}\right\rangle\right.\right.\right.\right.$ of $2 k-1$ consecutive, non-overlapping intervals with $k \geq 1, T_{i, 0}=$ $T_{i}$. and $T_{i, k}=T_{i}^{\prime}$. Naturally, if $\phi$ is a $\mathrm{MITL}_{0, \infty}$ formula and $\sigma^{\prime}$ is a refinement of $\sigma$, then $\sigma^{\prime} \models \phi$ iff $\sigma \models \phi$.

Taking an arbitrary trace $\sigma$, it may happen that the value of a compound sub-formula changes within an open interval. To capture the desired case when this does not happen, we call $\sigma$ fine for a formula $\phi$ (or $\phi$-fine) if for each sub-formula $\psi$ of $\phi$ (including $\phi$ itself), for each interval $I_{i}$ in $\sigma$, and for all $t, t^{\prime} \in I_{i}$, it holds that $\sigma^{(i, t)} \models \psi$ iff $\sigma^{\left(i, t^{\prime}\right)} \models \psi$.

Example 2: The following super-dense timed trace $\sigma=$ $\left\langle\begin{array}{c}{[0,0]} \\ \{p\}\end{array}\right\rangle\left\langle\begin{array}{c}(0,4.1) \\ \{p\}\end{array}\right\rangle\left\langle\begin{array}{c}{[4.1,4.1]} \\ \{p\}\end{array}\right\rangle\left\langle\begin{array}{c}{[4.1,4.1]} \\ \{q\}\end{array}\right\rangle\left\langle\begin{array}{c}{[4.1,4.1]} \\ \emptyset\end{array}\right\rangle \ldots$ is not fine for $\mathbf{G}_{\leq 1}^{\mathrm{s}} p$ as, e.g., (i) $\sigma^{(1, t)} \models \mathbf{G}_{\leq 1}^{\mathrm{s}} p$ for all $0 \leq t<3.1$ but (ii) $\sigma^{(1, t)} \not \models \mathbf{G}_{\leq 1}^{\mathrm{s}} p$ for all $3.1 \leq t<4$.1. We can make the beginning of the trace $\mathbf{G}_{\leq 1}^{\mathrm{s}} p$-fine by refining it to $\left\langle\begin{array}{c}{[0,0]} \\ \{p\}\end{array}\right\rangle\left\langle\begin{array}{c}(0,3.1) \\ \{p\}\end{array}\right\rangle\left\langle\begin{array}{c}{[3.1,3.1]} \\ \{p\}\end{array}\right\rangle\left\langle\begin{array}{c}(3.1,4.1) \\ \{p\}\end{array}\right\rangle\left\langle\begin{array}{c}{[4.1,4.1]} \\ \{p\}\end{array}\right\rangle \ldots$

By definition, every trace $\sigma$ is fine for each atomic proposition $p \in A P$. Furthermore, if $\sigma$ is $\phi$-fine and $\psi$ fine, then it is also fine for $\neg \phi, \phi \wedge \psi$, and $\phi \vee \psi$. For temporal operators $\mathbf{U}_{\bowtie n}^{\mathrm{s}}$ and $\mathbf{R}_{\bowtie n}^{\mathrm{s}}$, we have the following lemma stating that their values can change only once during an open interval given the trace is fine for the sub-formulas:

Lemma 1: If a trace $\sigma$ is fine for $\phi$ and $\psi, i \in \mathbb{N}, t, u \in I_{i}$, $\triangleleft \in\{<, \leq\}$, and $\triangleright \in\{\geq,>\}$, then

- if $\sigma^{(i, t)} \models \phi \mathbf{U}_{\triangleleft n}^{\mathrm{s}} \psi$ and $u \geq t$, then $\sigma^{(i, u)} \models \phi \mathbf{U}_{\triangleleft n}^{\mathrm{s}} \psi$;

- if $\sigma^{(i, t)} \models \phi \mathbf{U}_{\triangleright n}^{\mathrm{s}} \psi$ and $u \leq t$, then $\sigma^{(i, u)}=\phi \mathbf{U}_{\triangleright n}^{\mathrm{s}} \psi$;

- if $\sigma^{(i, t)} \models \phi \mathbf{R}_{\triangleleft n}^{\mathrm{s}} \psi$ and $u \leq t$, then $\sigma^{(i, u)} \models \phi \mathbf{R}_{\triangleleft n}^{\mathrm{s}} \psi$;

- if $\sigma^{(i, t)} \models \phi \mathbf{R}_{\triangleright n}^{\mathrm{s}} \psi$ and $u \geq t$, then $\sigma^{(i, u)}=\phi \mathbf{R}_{\triangleright n}^{\mathrm{s}} \psi$.

Thus, if $\sigma$ is fine for two formulas, it can be made fine for their compound by splitting each open interval at most once.

Lemma 2: Let $\phi$ be a $\operatorname{MITL}_{0, \infty}$ formula and $\sigma$ a trace. There is a refinement $\sigma^{\prime}$ of $\sigma$ that is $\phi$-fine. Such a refinement can be obtained by splitting each open interval in $\sigma$ into at most $2^{K}$ new open intervals and $2^{K}-1$ singletons, where $K$ is the number of timed until and release operators in $\phi$.

\section{Timed Automata Runs as Super-Dense Timed Traces}

We now describe the relationship between timed automata runs and super-dense timed traces. In our theory part, when model checking timed automata with $\mathrm{MITL}_{0, \infty}$, we assume that the atomic propositions only concern locations of the automaton. That is, they are of form "@ $l_{i}$ ", where $l_{i}$ is a location in the automaton. Of course, in the practice when compositions of timed automata with discrete local variables are handled, the atomic propositions can be more complex. However, we do assume that the atomic propositions do not change their values during the time elapse steps.

Consider a run $\pi=\left\langle l_{0}, \nu_{0}\right\rangle \stackrel{\delta_{0}}{\longrightarrow}\left\langle l_{1}, \nu_{1}\right\rangle \stackrel{\delta_{1}}{\longrightarrow} \ldots$ of a timed automaton $\mathcal{A}$. For each $\left\langle l_{i}, \nu_{i}\right\rangle$ in $\pi$ let $t_{i}=\sum_{j=0}^{i-1} \delta_{j}$ be the cumulative time spent in the run before the state, i.e. $t_{i}$ is "the time when the state occurs in $\pi$ ". Thus, at the time point $t_{i}$ the automaton is in the state $\left\langle l_{i}, \nu_{i}\right\rangle$ and we shall have $\left\langle\begin{array}{l}{\left[t_{i}, t_{i}\right]} \\ \left\{@ l_{i}\right\}\end{array}\right\rangle$ in the corresponding timed trace. The time elapse steps in the run produce the missing open intervals: when $\left\langle l_{i}, \nu_{i}\right\rangle \stackrel{\delta_{i}}{\longrightarrow}\left\langle l_{i+1}, \nu_{i+1}\right\rangle$ with $\delta_{i}>0$ (and thus $l_{i}=l_{i+1}$ ), then an open interval element $\left\langle\begin{array}{c}\left(t_{i}, t_{i+1}\right) \\ \left\{@ l_{i}\right\}\end{array}\right\rangle$ lies in between $\left\langle\begin{array}{c}{\left[t_{i}, t_{i}\right]} \\ \left\{@ l_{i}\right\}\end{array}\right\rangle$ and $\left\langle\begin{array}{c}{\left[t_{i+1}, t_{i+1}\right]} \\ \left\{@ l_{i}\right\}\end{array}\right\rangle$ in the timed trace.

Example 3: The run $\left\langle l_{1},(0,0)\right\rangle \stackrel{3.5}{\longrightarrow}\left\langle l_{1},(3.5,3.5)\right\rangle \stackrel{0}{\rightarrow}$ $\left\langle l_{2},(3.5,0)\right\rangle \stackrel{0}{\rightarrow}\left\langle l_{3},(3.5,0)\right\rangle \stackrel{1.1}{\rightarrow}\left\langle l_{3},(4.6,1.1)\right\rangle \ldots$ of the automaton in Figure 1 corresponds to the trace $\sigma=$ $\left\langle\begin{array}{l}{[0,0]} \\ \left\{@ l_{1}\right\}\end{array}\right\rangle\left\langle\begin{array}{c}(0,3.5) \\ \left\{@ l_{1}\right\}\end{array}\right\rangle\left\langle\begin{array}{c}{[3.5,3.5]} \\ \left\{@ l_{1}\right\}\end{array}\right\rangle\left\langle\begin{array}{c}{[3.5,3.5]} \\ \left\{@ l_{2}\right\}\end{array}\right\rangle\left\langle\begin{array}{c}{[3.5,3.5]} \\ \left\{@ l_{3}\right\}\end{array}\right\rangle\left\langle\begin{array}{c}\left(\begin{array}{c}3.5,4.6) \\ \left\{@ l_{3}\right\}\end{array}\right.\end{array}\right\rangle \ldots$

Recall that we will need to consider certain refinements of timed traces when model checking with $\mathrm{MITL}_{0, \infty}$ formulas. All the refinements of a timed trace produced by a timed automata run can be produced by other runs of the same automaton. That is, considering a trace coming from a run $\pi=\left\langle l_{0}, \nu_{0}\right\rangle \stackrel{\delta_{0}}{\longrightarrow}\left\langle l_{1}, \nu_{1}\right\rangle \stackrel{\delta_{1}}{\longrightarrow} \ldots$ of a timed automaton, each refinement can be obtained by considering the corresponding run $\pi^{\prime}$ where each time elapse step $\left\langle l_{i}, \nu_{i}\right\rangle \stackrel{\delta_{i}}{\longrightarrow}\left\langle l_{i+1}, \nu_{i+1}\right\rangle$ in $\pi$, with $\delta_{i}>0$ and $l_{i+1}=l_{i}$, is split into a sequence $\left\langle l_{i}, \nu_{i}\right\rangle \stackrel{\delta_{i, 1}}{\longrightarrow}\left\langle l_{i}, \nu_{i, 1}\right\rangle \stackrel{\delta_{i, 2}}{\longrightarrow} \ldots \stackrel{\delta_{i, k}}{\longrightarrow}\left\langle l_{i}, \nu_{i, k}\right\rangle$ of time elapse steps such that $\sum_{1 \leq j \leq k} \delta_{i, j}=\delta_{i}$ (and thus $\nu_{i, k}=\nu_{i+1}$ ).

\section{Symbolic ENCODING OF Timed Traces}

We now describe how to symbolically represent systems producing super-dense timed traces. The symbolical representation intended not as a replacement for timed automata but as a foundation for their symbolic verification, i.e. it is intended for use in the "back-end" of the verification tool and not as a modeling language. After the formalism is introduced, it will be shown how timed automata can be represented in this framework. The next section will then address the question of how to encode $\mathrm{MITL}_{0, \infty}$ formulas in this framework so that they are symbolically evaluated. Finally, in Sect. VI it will be demonstrated how finite versions of these encodings can be obtained by using region abstraction, allowing us to perform actual symbolic model checking of $\mathrm{MITL}_{0, \infty}$ formulas on timed automata.

\section{A. Symbolic Transition Systems with Clock-like Variables}

In the following, we use standard concepts of propositional and first-order logics, and assume that the formulas are interpreted modulo some background theory such as linear arithmetics (see e.g. [10] and the references therein). Given a set of typed variables, a valuation $v$ over the set is a function 
that assigns each variable in the set a value in the domain of the variable. We use $v \models \phi$ to denote that $v$ evaluates a quantifier-free formula $\phi$ over the set to true.

A symbolic transition system with clock-like variables, for brevity simply referred to as a transition system for the remainder of the paper, over a set $A P$ of atomic propositions is a tuple $\langle Z, X, \mathcal{I}, \mathcal{I} \mathcal{N} \mathcal{V}, \mathcal{T}, \mathcal{F}, \widehat{A P}\rangle$, where

- $Z=\left\{z_{1}, \ldots, z_{n}\right\}$ is a set of typed non-clock variables, $Z^{\prime}=\left\{z_{1}^{\prime}, \ldots, z_{n}^{\prime}\right\}$ being their next-state versions,

- $X=\left\{x_{1}, \ldots, x_{m}\right\}$ is a set of non-negative real-valued clock variables, $X^{\prime}=\left\{x_{1}^{\prime}, \ldots, x_{m}^{\prime}\right\}$ again being their next-state versions,

- $\mathcal{I}$ is the initial state formula over $Z \cup X$,

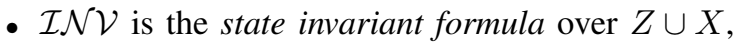

- $\mathcal{T}$ is the transition relation formula over $Z \cup X \cup\{\delta\} \cup$ $Z^{\prime} \cup X^{\prime}$, with a real-valued duration variable $\delta$,

- $\mathcal{F}$ is a finite set of fairness formulas over $Z$, and

- $\widehat{A P}$ associates each atomic proposition $p \in A P$ with a corresponding formula $\hat{p}$ over $Z$.

To ensure that the clock variables are used properly, we require that all the atoms in all the formulas in the system follow these rules: (i) if a non-clock variable in $Z$ or in $Z^{\prime}$ occurs in the atom, then none of the variables in $X \cup X^{\prime} \cup\{\delta\}$ occur in it, and (ii) if a variable in $X \cup X^{\prime} \cup\{\delta\}$ occurs in it, then it is of the forms $x^{\prime}=0, x^{\prime}=x+\delta, x \bowtie n, x+\delta \bowtie n$, or $\delta \bowtie 0$ where $\bowtie \in\{<, \leq,=, \geq,>\}, x, x^{\prime} \in X$ and $n \in \mathbb{N}$. Furthermore, for all valuations $\tau$ over $Z \cup X \cup\{\delta\} \cup Z^{\prime} \cup X^{\prime}$ such that $\tau \models \mathcal{T}$, it must hold that $\tau(\delta) \geq 0$ and for each clock $x \in X$ either $\tau\left(x^{\prime}\right)=0$ or $\tau\left(x^{\prime}\right)=\tau(x)+\tau(\delta)$.

A state of the system now is a valuation $s$ over $Z \cup X$ and a run an infinite sequence $s_{0} \stackrel{\delta_{0}}{\longrightarrow} s_{1} \stackrel{\delta_{1}}{\longrightarrow} s_{2} \ldots$ such that

- $\delta_{0}=0$ and for all $i \in \mathbb{N}$ we have $\delta_{i} \geq 0, s_{i}(x) \geq 0$ when $x \in X$, and $\delta_{i}>0 \Rightarrow \delta_{i+1}=0$,

- $s_{0}=\mathcal{I}$ and $s_{i}=\mathcal{I} \mathcal{N} \mathcal{V}$ holds for all $i \in \mathbb{N}$,

- for all $i \in \mathbb{N}$ it holds that $\left\{y \mapsto s_{i}(y) \mid y \in Z \cup X\right\} \cup$ $\left\{\delta \mapsto \delta_{i}\right\} \cup\left\{y^{\prime} \mapsto s_{i+1}(y) \mid y \in Z \cup X\right\} \models \mathcal{T}$, and

- for each $f \in \mathcal{F}$, there are infinitely many states $s$ in the run for which $s \models f$ holds.

A run $\tau=s_{0} \stackrel{\delta_{0}}{\longrightarrow} s_{1} \stackrel{\delta_{1}}{\longrightarrow} s_{2} \stackrel{\delta_{2}}{\longrightarrow} \ldots$ represents the superdense timed trace $\operatorname{trace}(\tau)=\left\langle I_{0}, v_{0}\right\rangle\left\langle I_{1}, v_{1}\right\rangle\left\langle I_{2}, v_{2}\right\rangle \ldots$ over $A P$ where for each $i \in \mathbb{N}$,

- $v_{i}=\left\{p \in A P \mid s_{i} \models \hat{p}\right\}$, and

- letting $t_{i}=\sum_{j=0}^{i-1} \delta_{j}$, (i) if $\delta_{i}=0$, then $I_{i}=\left[t_{i}, t_{i}\right]$, and (ii) if $\delta_{i}>0$, then $I_{i}=\left(t_{i}, t_{i}+\delta_{i}\right)$.

The set of all traces of a transition system $\mathcal{S}$ is $\operatorname{traces}(\mathcal{S})=$ $\{\operatorname{trace}(\tau) \mid \tau$ is a run of $\mathcal{S}\}$. The transition system $\mathcal{S}$ is refinement-admitting if $\sigma \in \operatorname{traces}(\mathcal{S})$ implies $\sigma^{\prime} \in$ $\operatorname{traces}(\mathcal{S})$ for all the refinements $\sigma^{\prime}$ of $\sigma$.

\section{B. Encoding Timed Automata Traces}

Recall the correspondence between timed automata runs and traces discussed in Sect. III-C. Given a timed automaton
$\mathcal{A}=\left\langle L, l_{\text {init }}, X, E, I\right\rangle$, we can encode it as a transition system $\mathcal{S}_{\mathcal{A}}=\langle Z, X, \mathcal{I}, \mathcal{I} \mathcal{N} \mathcal{V}, \mathcal{T}, \emptyset, \widehat{A P}\rangle$, where ${ }^{2}$

- $Z=\{a t\}$, where at is a variable with the domain $L$,

- $\mathcal{I}:=\left(a t=l_{\text {init }}\right) \wedge \bigwedge_{x \in X}(x=0)$,

- $\mathcal{I N} \mathcal{V}:=\bigwedge_{l \in L}(a t=l) \Rightarrow I(l)$

- $\mathcal{T}:=\left(\left(\delta=0 \wedge \delta^{\prime}=0\right) \Rightarrow \bigvee_{\left\langle l, g, r, l^{\prime}\right\rangle \in E} a t=l \wedge a t^{\prime}=l^{\prime}\right.$ $\left.\wedge g \wedge\left(\bigwedge_{x \in R} x^{\prime}=0\right) \wedge\left(\bigwedge_{x \in X \backslash R} x^{\prime}=x\right)\right)$

$\wedge\left(\left(\delta>0 \vee \delta^{\prime}>0\right) \Rightarrow\left(a t^{\prime}=a t \wedge \bigwedge_{x \in X} x^{\prime}=x+\delta\right)\right)$ $\wedge\left(\delta=0 \vee \delta^{\prime}=0\right)$

(Recall that $\delta$ special real-valued duration variable)

- $\widehat{A P}$ associates each atomic proposition @ $l$, where $l \in$ $L$, with the formula $(a t=l)$.

Now traces $\left(\mathcal{S}_{\mathcal{A}}\right)$ is exactly the set of super-dense timed traces corresponding to the runs of the automaton $\mathcal{A}$. Every state of $\mathcal{S}_{\mathcal{A}}$ corresponds to a time interval in the timed trace of $\mathcal{A}$. Thus, there are three types of transitions encoded in $\mathcal{T}$. Firstly, a singleton-to-singleton transition, corresponding to a discrete transition of $\mathcal{A}$, occurs when $\delta$ and $\delta^{\prime}$ are both zero. Secondly, a singleton-to-open transition occurs when the $\delta$ is zero and $\delta^{\prime}$ non-zero. On such a transition, all variables remain unchanged. Hence, the clocks values correspond to the left bound of the interval. Thirdly, on a open-to-singleton transition $\left(\delta>0\right.$ and $\left.\delta^{\prime}=0\right)$ the clock variables are updated according to the length of the open interval.

Due to the "repetition of time elapse steps" property of timed automata discussed in Sect. III-C, the transition system $\mathcal{S}_{\mathcal{A}}$ is also refinement-admitting.

\section{Symbolic ENCODING OF MITL $_{0, \infty}$ FORMULAS}

Let $\mathcal{S}=\langle Z, X, \mathcal{I}, \mathcal{I} \mathcal{N} \mathcal{V}, \mathcal{T}, \mathcal{F}, \widehat{A P}\rangle$ be a transition system over $A P$ encoding some timed system producing super-dense timed traces. We now augment $\mathcal{S}$ with new variables and constraints so that $\mathrm{MITL}_{0, \infty}$ formulas over $A P$ are symbolically evaluated in the runs of the transition systems. We say that the resulting transition system $\mathcal{S}_{\phi}=\left\langle Z \cup Z_{\phi}, X \cup X_{\phi}, \mathcal{I} \wedge \mathcal{I}_{\phi}, \mathcal{I} \mathcal{N} \mathcal{V}, \mathcal{T} \wedge \mathcal{T}_{\phi}, \mathcal{F} \cup \mathcal{F}_{\phi}, \widehat{A P}\right\rangle$ over $A P$ encodes $\phi$ if $Z_{\phi}$ includes a Boolean variable $\|\psi\|$ for each sub-formula $\psi$ of $\phi$ (including $\phi$ itself). Furthermore, we require two conditions on such encodings.

First, we want to make sure that the encoding $\mathcal{S}_{\phi}$ is sound in the following senses:

- all the traces of $\mathcal{S}$ (i.e, projections of runs to the atomic propositions) are preserved: $\operatorname{traces}\left(\mathcal{S}_{\phi}\right)=\operatorname{traces}(\mathcal{S})$

- when $\phi$ is holds in a state, then it holds in the corresponding interval: for each run $\tau=s_{0} s_{1} \ldots$ of $\mathcal{S}_{\phi}$ with $\operatorname{trace}(\tau)=\sigma=\left\langle I_{0}, v_{0}\right\rangle\left\langle I_{1}, v_{1}\right\rangle \ldots$, and each $i \in \mathbb{N}, s_{i}(\|\phi\|)=$ true implies $\forall t \in I_{i}: \sigma^{(i, t)} \models \phi$.

For fine traces we want to faithfully capture the cases when a formula holds on some interval. To this end, we say that

\footnotetext{
${ }^{2}$ Strictly, the atoms $\delta^{\prime}=0$ and $\delta^{\prime}>0$ are not allowed in $\mathcal{T}$; this can be handled by adding new Boolean variables $\delta=0$ and $\delta>0$ in $Z$, forcing $\underline{\delta=0} \Rightarrow(\delta=0)$ and $\delta>0 \Rightarrow(\delta>0)$ in $\mathcal{T}$, and then using $\underline{\delta=0}$ instead of $\delta^{\prime}=0$ and $\delta>0^{\prime}$ instead of $\delta^{\prime}>0$ in the rest of $\mathcal{T}$.
} 
the encoding $\mathcal{S}_{\phi}$ is complete if for every $\phi$-fine trace $\sigma=$ $\left\langle I_{0}, v_{0}\right\rangle\left\langle I_{1}, v_{1}\right\rangle\left\langle I_{2}, v_{2}\right\rangle \ldots$ in traces $(\mathcal{S})$, there is a run $\tau=$ $s_{0} s_{1} s_{2} \ldots$ in $\mathcal{S}_{\phi}$ such that $\operatorname{trace}(\tau)=\sigma$ and for all points $(i, t)$ in $\sigma$ it holds that $\sigma^{(i, t)} \models \phi$ implies $v_{i}(\|\phi\|)=$ true.

Therefore, our model checking task "Does a refinementadmitting transition system $\mathcal{S}$ have a run corresponding to a trace $\sigma$ with $\sigma=\phi$ ?" is reduced to the problem of deciding whether $\mathcal{S}_{\phi}$ has a run $s_{0} s_{1} s_{2} \ldots$ with $s_{0}(\|\phi\|)=$ true.

\section{A. Encoding Propositional Subformulas}

Let $\mathcal{S}=\langle Z, X, \mathcal{I}, \mathcal{I N} \mathcal{V}, \mathcal{T}, \mathcal{F}, \widehat{A P}\rangle$ be a transition system over $A P$. For the atomic formulas $\phi$ of forms $p$ and $\neg p$, it is possible to make a transition system $\mathcal{S}_{\phi}=$ $\left\langle Z \cup\{\|p\|\}, X, \mathcal{I}, \mathcal{I} \mathcal{N} \mathcal{V}, \mathcal{T} \wedge \mathcal{T}_{\phi}, \mathcal{F}, \widehat{A P}\right\rangle$ encoding $\phi$ by (i) defining $\mathcal{T}_{\phi}:=(\|\phi\| \Leftrightarrow \hat{p})$ if $\phi=p$ and (ii) $\mathcal{T}_{\phi}:=(\|\phi\| \Leftrightarrow$ $\neg \hat{p})$ if $\phi=\neg p$. Similarly, assuming that $\phi$ is either of form $\alpha \wedge \beta$ or $\alpha \vee \beta$ for some MITL $_{0, \infty}$ formulas $\alpha$ and $\beta$, and that $\mathcal{S}$ encodes both $\alpha$ and $\beta$, we can make a transition system $\mathcal{S}_{\phi}=\left\langle Z \cup\{\|p\|\}, X, \mathcal{I}, \mathcal{I} \mathcal{N} \mathcal{V}, \mathcal{T} \wedge \mathcal{T}_{\phi}, \mathcal{F}, \widehat{A P}\right\rangle$ encoding $\phi$ as follows: (i) if $\phi=\alpha \vee \beta$, then $\mathcal{T}_{\phi}:=(\|\phi\| \Leftrightarrow(\|\alpha\| \vee\|\beta\|))$, and, (ii) if $\phi=\alpha \wedge \beta$, then $\mathcal{T}_{\phi}:=(\|\phi\| \Leftrightarrow(\|\alpha\| \wedge\|\beta\|))$.

The lemmas for the soundness and completeness of the encodings are given in Sect. V-C.

\section{B. Encoding MITL $_{0, \infty}$ operators}

In the following sub-sections, we present encodings for the other $\mathrm{MITL}_{0, \infty}$ operators. In each encoding, we may introduce some new non-clock and clock variables such as $c$ and lefto; these variables are "local" to the encoded subformula $\psi$ and not used elsewhere, we do not subscript them (e.g. $c$ really means $c_{\psi}$ ) for the sake of readability. We also introduce new transition relation constraints (i.e. conjuncts in $\mathcal{T}_{\psi}$ ), initial state constraints and fairness conditions. We will use open as a shorthand for $(\delta>0)$.

1) Encoding $l \mathbf{U}_{\triangleleft n}^{\mathrm{s}} r$ and $l \mathbf{R}_{\triangleleft n}^{\mathrm{s}} r$ with $\triangleleft \in\{<, \leq\}$ : These operators can be expressed with simpler ones by using the following lemma (proven in the appendix):

Lemma 3: $\sigma^{(i, t)}=\phi \mathbf{U}_{\triangleleft n}^{\mathrm{s}} \psi$ iff $\sigma^{(i, t)}=\left(\mathbf{F}_{\triangleleft n}^{\mathrm{s}} \psi\right) \wedge\left(\phi \mathbf{U}^{\mathrm{s}}\right.$ $\psi$ ) for all $i \in \mathbb{N}, t \in I_{i}, \triangleleft \in\{<, \leq\}$, and $n \in \mathbb{N}$.

Using the $\mathbf{U}_{\triangleleft n}^{\mathrm{s}} / \mathbf{R}_{\triangleleft n}^{\mathrm{s}}$ duality, we can now also express $\phi \mathbf{R}_{\triangleleft n}^{\mathrm{s}} \psi$ as $\left(\mathbf{G}_{\triangleleft n}^{\mathrm{s}} \psi\right) \wedge\left(\phi \mathbf{R}^{\mathrm{s}} \psi\right)$.

2) Encoding $l \mathbf{U}^{\mathrm{s}} r$ : We encode "untimed" until formulas $l \mathbf{U}^{\mathrm{s}} r$ essentially like in the traditional LTL case [11] but must consider open intervals and singletons separately.

Assume $l \mathbf{U}^{\mathrm{s}} r$ holds on the current interval. If that interval is open, $l$ and one of the following hold: (i) $r$ holds on the current interval, (ii) $r$ holds on the next interval (which is a singleton), or (iii) $l$ holds on the next interval and $l \mathbf{U}^{\mathrm{s}} r$ is satisfied as well. This is captured by the following constraint:

$$
\left.\left.\left.\left.\left\|l \mathbf{U}^{\mathrm{s}} r\right\| \wedge \text { open } \Rightarrow \| l\right]\left\|\wedge(\|r\| \vee \| r]^{\prime} \vee(\| l]^{\prime} \wedge\right\| l \mathbf{U}^{\mathrm{s}} r\right]^{\prime}\right)\right)
$$

If, in contrast, the current interval is a singleton, then there are two possibilities: (i) the next interval is a singleton and

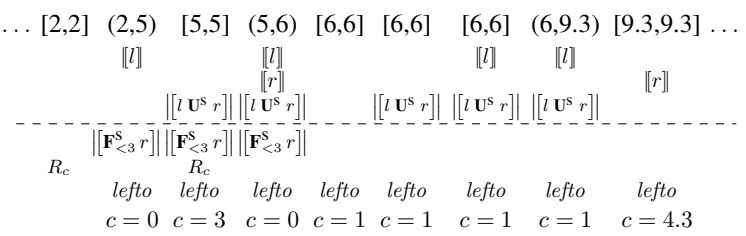

Figure 2. Encoding $l \mathbf{U}^{\mathrm{s}} r$ and $\mathbf{F}_{<3}^{\mathrm{s}} r$

$r$ holds, or (ii) both $l$ and $l \mathbf{U}^{\mathrm{s}} r$ hold on the next interval:

$$
\left.\left\|l \mathbf{U}^{\mathrm{s}} r\right\| \wedge \neg \text { open } \Rightarrow\left(\neg \text { open }^{\prime} \wedge\|r\|^{\prime}\right) \vee\left(\|l\|^{\prime} \wedge \| l \mathbf{U}^{\mathrm{s}} r\right]^{\prime}\right)
$$

Finally, as in the traditional LTL encoding, we must add a fairness condition in order to avoid the case where $\left\|l \mathbf{U}^{\mathrm{s}} r\right\|$ and $\|l\|$ are true on all intervals starting from some point but $r$ does not hold at any future time point, i.e. $\mathcal{F}_{l \mathbf{U}^{\mathrm{s}} r}=$ $\left\{\neg\left\|l \mathbf{U}^{\mathrm{s}} r\right\| \vee\|r\|\right\}$.

Example 4: Figure 2 illustrates an evaluation of the encoding variables on a trace (ignore the text below the dashed line for now). Note that $\left\|l \mathbf{U}^{\mathrm{s}} r\right\|$ is (correctly) evaluated to true on the second $[6,6]$-interval despite $l$ not holding.

3) Encoding $\mathbf{F}_{\leq 0}^{\mathrm{s}} r$ : A formula $\mathbf{F}_{\leq 0}^{\mathrm{s}} r$ holding requires a future interval at which $r$ holds and which can be reached without any time passing. Thus, $\mathbf{F}_{\leq 0}^{\mathrm{s}} r$ is satisfied only on a singleton where the next interval is a singleton as well and (i) $r$ or (ii) $\mathbf{F}_{\leq 0}^{\mathrm{s}} r$ holds on the next interval:

$$
\left.\left.\left|\left[\mathbf{F}_{\leq 0}^{\mathrm{s}} r\right]\right| \Rightarrow \neg \text { open } \wedge \neg \text { open }^{\prime} \wedge(\| r]^{\prime} \vee \mid\left[\mathbf{F}_{\leq 0}^{\mathrm{s}} r\right]\right]^{\prime}\right)
$$

No fairness conditions are needed as the non-zenoness requirement always guarantees a future open interval.

4) Encoding $\mathbf{F}_{\triangleleft n}^{\mathrm{s}} r$ with $n>0$ : In the encoding of $\mathbf{F}_{\triangleleft n}^{\mathrm{s}}$, we first add the constraints for $\mathbf{U}^{\mathrm{s}}$ replacing $l$ by true.

$$
\begin{aligned}
\left\|\mathbf{F}_{\triangleleft n}^{\mathrm{s}} r\right\| \wedge \text { open } & \left.\Rightarrow\|r\| \vee\|r\|^{\prime} \vee \| \mathbf{F}_{\triangleleft n}^{\mathrm{s}} r\right]^{\prime} \\
\left\|\mathbf{F}_{\triangleleft n}^{\mathrm{s}} r\right\| \wedge \neg \text { open } & \left.\Rightarrow\|r\|^{\prime} \vee \| \mathbf{F}_{\triangleleft n}^{\mathrm{s}} r\right]^{\prime}
\end{aligned}
$$

Next, we observe that for encoding timing related aspect, it is sufficient to at any point remember the earliest interval at which $\mathbf{F}_{\triangleleft n}^{\mathrm{s}} r$ holds and after which $r$ has not held yet. If $r$ is encountered in time for the earliest such interval, then interval where $r$ holds is close enough to any later interval where $\left\|\mathbf{F}_{\triangleleft n}^{\mathrm{s}} r\right\|$ holds as well. Correspondingly, we use a real-valued (clock-like) auxiliary variable $c$ and a boolean auxiliary variable lefto to remember the time passed since and type of the earliest interval on which $\left\|\mathbf{F}_{\triangleleft n}^{\mathrm{s}} r\right\|$ held and after which we have not seen $\|r\|$. The correct values in the first interval are forced by the initial state formula $\mathcal{I}_{\mathbf{F}_{\triangleleft n}^{s} r}:=c=0 \wedge \neg$ lefto. To update $c$ and lefto, we define the shorthand $R_{c}$ to be true when we have not seen $\left\|\mathbf{F}_{\triangleleft n}^{\mathrm{s}} r\right\|$ without seeing $r$ afterwards or $r$ holds on an open current or an arbitrary next interval.

$$
\left.\left.R_{c}:=\left(\neg\left\|\mathbf{F}_{\triangleleft n}^{\mathrm{s}} r\right\| \vee(\text { open } \wedge\|r\|) \vee \| r\right]^{\prime}\right) \wedge \| \mathbf{F}_{\triangleleft n}^{\mathrm{s}} r\right]^{\prime}
$$


We then (i) reset $c$ and lefto on the next interval if $R_{c}$ holds on the current interval, and (ii) update $c$ and leave lefto unchanged if $R_{c}$ does not hold.

$$
\begin{aligned}
& R_{c} \Rightarrow c^{\prime}=0 \wedge\left(\text { lefto }^{\prime} \Leftrightarrow \text { open }^{\prime}\right) \\
& \neg R_{c} \Rightarrow c^{\prime}=c+\delta \wedge\left(\text { lefto }^{\prime} \Leftrightarrow \text { lefto }\right)
\end{aligned}
$$

We introduce a shorthand $T_{c}$ (defined below) such that $T_{c}$ holds if for each point on the interval where we reset $c$ there is a point on the next interval that satisfies the $\triangleleft n$ constraint. We then require that $\left\|\mathbf{F}_{\triangleleft n}^{\mathrm{s}} r\right\|$ being true, and $r$ being false or the current interval being a singleton implies that $T_{c}$ holds.

$$
\left.\left(\left\|\mathbf{F}_{\triangleleft n}^{\mathrm{s}} r\right\| \wedge \neg(\| r] \wedge \wedge \text { open }\right)\right) \Rightarrow T_{c}
$$

In the case of $\mathbf{F}_{<n}^{\mathrm{s}} r$, we define $T_{c}:=c+\delta<n \vee$ (lefto $\wedge c+\delta \leq n)$ and in the case of $\mathbf{F}_{<n}^{\mathrm{s}} r$ we define $T_{c}:=c+\delta<n \vee\left(\left(\neg\right.\right.$ open $^{\prime} \vee$ lefto $\left.) \wedge c+\bar{\delta} \leq n\right)$.

Example 5: An evaluation of the encoding variables is shown (below the dashed line) in Figure 2. Especially, observe that $\left\|\mathbf{F}_{>3}^{\mathrm{s}} r\right\|$ is not evaluated to true on the interval $(6,9.3)$ although $\mathbf{F}_{>3}^{\mathrm{s}} r$ holds on some points in the interval: we are interested in sound encodings and $\left\|\mathbf{F}_{>3}^{\mathrm{s}} r\right\|$ does not hold on all the points in the interval.

5) Encoding $l \mathbf{U}_{\triangleright n}^{\mathrm{s}} r$ with $\triangleright \in\{\geq,>\}$ : To encode $l \mathbf{U}_{\triangleright n}^{\mathrm{s}}$ $r$, we define shorthands $T_{c}$ and $\hat{r}$. $T_{c}$ will later be defined so that $T_{c}$ holds iff for every previous point at which $\left\|l \mathbf{U}_{\triangleright n}^{\mathrm{s}} r\right\|$ held there is a point on the current interval that satisfies the $\triangleright n$ timing constraint. We, then, define $\hat{r}:=\|r\| \wedge T_{c}$. Next, we add a boolean "obligation" variable oblig to remember when we need to see $\hat{r}$ at a future point. Whenever $\left\|l \mathbf{U}_{\triangleright n}^{\mathrm{s}} r\right\|$ is true, we also require oblig to be true.

$$
\left\|l \mathbf{U}_{\triangleright n}^{\mathrm{s}} r\right\| \Rightarrow \text { oblig }
$$

In case $n>0$, we additionally require oblig and $l$ to hold on the next interval.

$$
\left\|l \mathbf{U}_{\triangleright n}^{\mathrm{s}} r\right\| \Rightarrow\left(\operatorname{oblig}^{\prime} \wedge l^{\prime}\right)
$$

Next, we add constraints similar to those for the $\mathbf{U}^{\mathrm{s}}$-operator but with $\left\|l \mathbf{U}_{\triangleright n}^{\mathrm{s}} r\right\|$ and $\|r\|$ replaced by oblig and $\hat{r}$.

$$
\begin{gathered}
(\text { oblig } \wedge \text { open }) \Rightarrow\left(\|l\| \wedge\left(\hat{r} \vee \hat{r}^{\prime} \vee\left(\|l\|^{\prime} \wedge \text { oblig }^{\prime}\right)\right)\right) \\
\left.(\text { oblig } \wedge \neg \text { open }) \Rightarrow\left(\left(\neg \text { open }{ }^{\prime} \wedge \hat{r}^{\prime}\right) \vee(\| l] \|^{\prime} \wedge \text { oblig }\right)\right)
\end{gathered}
$$

We want to determine whether the $\triangleright n$ constraint holds for all previous points at which $\left\|l \mathbf{U}_{\triangleright n}^{\mathrm{s}} r\right\|$ holds. We, thus, use a real-valued variable $c$ and a boolean variable righto to measure the time since the most recent corresponding interval. We, thus, reset $c$ to zero and use righto to remember the type of the current interval whenever $\left.\| l \mathbf{U}_{\triangleright n}^{\mathrm{s}} r\right] \|$ holds. Otherwise, we update $c$ and righto as before.

$$
\begin{aligned}
\left\|l \mathbf{U}_{\triangleright n}^{\mathrm{s}} r\right\| & \Rightarrow c^{\prime}=0 \wedge\left(\text { righto }^{\prime} \Leftrightarrow \text { open }\right) \\
\neg\left\|l \mathbf{U}_{\triangleright n}^{\mathrm{s}} r\right\| & \Rightarrow c^{\prime}=c+\delta \wedge\left(\text { righto }^{\prime} \Leftrightarrow \text { righto }\right)
\end{aligned}
$$

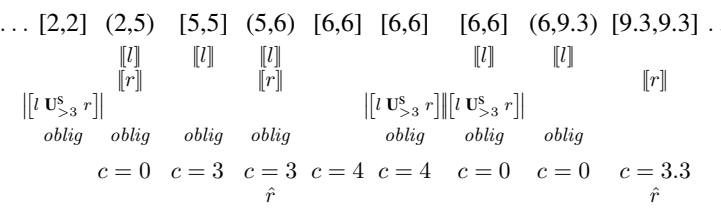

Figure 3. Encoding $l \mathbf{U}_{>3}^{\mathrm{s}} r$

Next, in case $l \mathbf{U}_{>n}^{\mathrm{s}} r$, we define $T_{c}:=c+\delta>n \vee$ (righto $\wedge c+\delta \geq n)$ and in case $l \mathbf{U}_{\geq n}^{\mathrm{s}} r$ we define $T_{c}:=$ $c+\delta>n \vee(($ righto $\vee \neg$ open $) \wedge c+\delta \geq n)$

Finally, as for the untimed $\mathbf{U}^{\mathrm{s}}$-operator, we need a fairness condition to prevent a situation where oblig holds globally but $r$ never holds. We define $\mathcal{F}_{l \mathbf{U}_{\triangleright n}^{s} r}:=\{\neg$ oblig $\left.\vee \| r]\right\}$. Note that, here, we use $\|r\|$, not $\hat{r}$. For instance, when $l \mathbf{U}_{\triangleright n}^{\mathrm{s}} r$ and $r$ hold globally, there may never be a point where $T_{c}$ is true and thus $\hat{r}$ always stays false.

Example 6: Figure 3 illustrates how the encoding variables of $l \mathbf{U}_{>3}^{\mathrm{s}} r$ variables could be evaluated on a trace. Again, $\left.\| l \mathbf{U}_{>3}^{\mathrm{s}} r\right]$ is not true on the interval $(6,9.3)$ because $l \mathbf{U}_{>3}^{\mathrm{s}} r$ holds only on some points on it but not on all.

6) Encoding $l \mathbf{R}^{\mathrm{s}} r$ : For encoding $l \quad \mathbf{R}^{\mathrm{s}} r$, we use an auxiliary boolean variable oblig. Intuitively, oblig being true means that before seeing any point at which $\|r\|$ is false, we need to see a point where $\| l]$ is true.

We require oblig to hold on the current interval when $\left\|l \mathbf{R}^{\mathrm{s}} r\right\|$ holds on an open interval and on the next interval when $\left\|l \mathbf{R}^{\mathrm{s}} r\right\|$ holds on a singleton.

$$
\begin{aligned}
& \left(\|\left[\mathbf{R}^{\mathrm{s}} r \| \wedge \text { open }\right) \quad \Rightarrow \quad\right. \text { oblig } \\
& \left(\left\|l \mathbf{R}^{\mathrm{s}} r\right\| \wedge \neg \text { open }\right) \quad \Rightarrow \quad \text { oblig }^{\prime}
\end{aligned}
$$

The obligation to see $l$ before $\neg r$ remains active until $l$ holds:

$$
\text { oblig } \Rightarrow\left(\|l\| \vee \text { oblig }^{\prime}\right)
$$

As a final constraint, $r$ needs to hold on all intervals where the obligation is true, with the exception of open intervals on which $l$ holds, leading to

$$
\text { oblig } \Rightarrow((\text { open } \wedge \| l] \|) \vee\|r\|)
$$

7) Encoding $\mathbf{G}_{\leq 0}^{\mathrm{s}} r: \mathbf{G}_{\leq 0}^{\mathrm{s}} r$ trivially holds when the current or the next interval is open. Furthermore, $\mathbf{G}_{\leq 0}^{\mathrm{s}} r$ holds when both current and next interval are singletons and $r$ and $\mathbf{G}_{\leq 0}^{\mathrm{s}} r$ hold on the next interval.

$$
\left.\left|\left[\mathbf{G}_{\leq 0}^{\mathrm{s}} r\right]\right| \Rightarrow\left(\text { open } \vee \text { open }^{\prime} \vee(\| r]^{\prime} \wedge\left|\left[\mathbf{G}_{\leq 0}^{\mathrm{s}} r\right]\right|^{\prime}\right)\right)
$$

8) Encoding $\mathbf{G}_{\triangleleft n}^{\mathrm{s}} r$ with $n>0$ : First, we require that $r$ holds on all open intervals on which $\left\|\mathbf{G}_{\triangleleft n}^{\mathrm{s}} r\right\|$ holds. Furthermore, we will later define a shorthand $T_{c}$ to hold whenever there is an interval on which $\left\|\mathbf{G}_{\triangleleft n}^{\mathrm{s}} r\right\|$ held sufficiently shortly in the past to still require $r$ to hold, resulting in

$$
\left(\left(\left\|\mathbf{G}_{\triangleleft n}^{\mathrm{s}} r\right\| \wedge \text { open }\right) \vee T_{c}\right) \Rightarrow\|r\|
$$




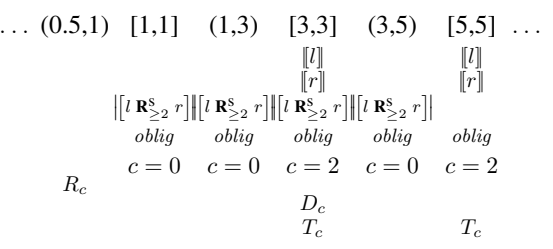

Figure 4. Encoding $l \mathbf{R}_{\geq 2}^{\mathrm{s}} r$.

Like in the $\mathbf{U}_{\triangleright}^{\mathrm{s}}$ encoding, we use a real-valued variable $c$ and a boolean variable righto to measure time from the most recent interval at which $\left\|\mathbf{G}_{\triangleleft n}^{\mathrm{s}} r\right\|$ held.

$$
\begin{aligned}
\left\|\mathbf{G}_{\triangleleft n}^{\mathrm{s}} r\right\| & \Rightarrow c^{\prime}=0 \wedge\left(\text { righto }^{\prime} \Leftrightarrow \text { open }\right) \\
\neg\left\|\mathbf{G}_{\triangleleft n}^{\mathrm{s}} r\right\| & \Rightarrow c^{\prime}=c+\delta \wedge\left(\text { righto }^{\prime} \Leftrightarrow \text { righto }\right)
\end{aligned}
$$

Now, in the case of $\mathbf{G}_{<n}^{\mathrm{s}}$ we define $T_{c}:=c<n$ and for $\mathbf{G}_{\leq n}^{\mathrm{s}}$ we define $T_{c}:=c<n \vee(c \leq n \wedge \neg$ open $\wedge \neg$ righto $)$

9) Encoding $l \mathbf{R}_{\triangleright n}^{\mathrm{s}} r$ : For encoding the lower bound until operators, we use a boolean variable oblig and the same update rules as for the untimed $\mathbf{R}^{\mathrm{s}}$ operator.

$$
\begin{array}{rll}
\left(\left\|l \mathbf{R}_{\triangleright n}^{\mathrm{s}} r\right\| \wedge \text { open }\right) & \Rightarrow & \text { oblig } \\
\left(\left\|l \mathbf{R}_{\triangleright n}^{\mathrm{s}} r\right\| \wedge \neg \text { open }\right) & \Rightarrow & \text { oblig }^{\prime} \\
\text { oblig } & \Rightarrow & \left.(\| l] \vee \text { oblig }^{\prime}\right)
\end{array}
$$

We add a modified version of Constraint 18 and use a shorthand $T_{c}$ (defined later) to identify intervals that contain time points $\triangleright n$ from a point where $\left.\| l \mathbf{R}_{\triangleright n}^{\mathrm{s}} r\right] \|$ holds.

$$
\left(\text { oblig } \wedge T_{c}\right) \Rightarrow((\|l\| \wedge \text { open }) \vee\|r\|)
$$

Next, we add a constraint for intervals of length $>n$. On such an interval, $l$ or $r$ has to hold if $\left\|l \mathbf{R}_{\triangleright n}^{\mathrm{s}} r\right\|$ holds.

$$
\left.\left(\| l \mathbf{R}_{\triangleright n}^{\mathrm{s}} r\right] \wedge \delta>n\right) \Rightarrow(\|l \rrbracket \vee\| r \|)
$$

For encoding $\mathbf{R}_{\triangleright n}^{\mathrm{s}}$, we use an auxiliary real-valued variable $c$ and a boolean variable lefto to measure the time passed since the earliest interval at which $\left.\| l \mathbf{R}_{\triangleright n}^{\mathrm{s}} r\right] \|$ holds and whose obligation to see $l$ before $r$ is still active. This is, in principle, similar to the $\mathbf{F}_{\triangleleft n}^{\mathrm{s}}$ encoding except for a special case illustrated in Figure 4. Here, on the fourth interval $c$ and lefto are needed for two purposes: to measure the time passed since the second interval (which introduced a still open obligation) and to start measuring time since the current interval (which introduces a fresh obligation as $\|l\|$ holds satisfying the previous obligation). We define a shorthand $D_{c}:=\left(\neg\right.$ open $\wedge$ oblig $\left.\left.\wedge l \wedge \| l \mathbf{R}_{\triangleright n}^{\mathrm{s}} r\right] \|\right)$ to captures precisely this situation and will later delay resetting $c$ by one step whenever $D_{c}$ holds. Otherwise, $c$ needs to be reset on the next interval if $\left.\| l \mathbf{R}_{\triangleright n}^{\mathrm{s}} r\right] \mid$ holds on that interval and (i) if there is an open obligation it is satisfied on the current interval and (ii) the current interval is not a singleton on which $\left.\| l \mathbf{R}_{\triangleright n}^{\mathrm{s}} r\right]$ holds, i.e. does not add an obligation to the next interval, i.e. $R_{c}:=\left\|l \mathbf{R}_{\triangleright n}^{\mathrm{s}} r\right\|^{\prime} \wedge(\neg$ oblig $\vee l) \wedge\left(\right.$ open $\vee \neg \|\left[l \mathbf{R}_{\triangleright n}^{\mathrm{s}} r \|\right)$.
As said before, we delay resetting $c$ and lefto by one interval when $D_{c}$ holds, i.e. set $c$ to 0 and lefto to false.

$$
D_{c} \Rightarrow\left(c^{\prime}=0 \wedge \neg \text { lefto }^{\prime}\right)
$$

When $R_{c}$ holds, $c$ and lefto are reset as for the $\mathbf{F}_{\triangleleft n}^{\mathrm{s}}$ operator and when neither holds we update them as usual:

$$
\begin{gathered}
R_{c} \Rightarrow\left(c^{\prime}=0 \wedge\left(\text { lefto }^{\prime} \Leftrightarrow \text { open }^{\prime}\right)\right) \\
\left(\neg R_{c} \wedge \neg D_{c}\right) \Rightarrow\left(c^{\prime}=c+\delta \wedge\left(\text { lefto }^{\prime} \Leftrightarrow \text { lefto }\right)\right)
\end{gathered}
$$

We set the initial values of $c$ and lefto to correspond measuring time from the initial interval, i.e. $\mathcal{I}_{l \mathbf{R}_{\triangleright n}^{\mathrm{s}} \|}\|r\|:=c=$ $0 \wedge \neg$ lefto.

Finally, we define $T_{c}$ to hold precisely if there is a point on the current interval that is $\triangleright n$ time units away from a point belonging to the interval at which we started measuring time. In the case of $\mathbf{R}_{>n}^{\mathrm{s}}$, we define $T_{c}:=c+\delta>n$ and for $\mathbf{R}_{\geq n}^{\mathrm{s}}$ we define $T_{c}:=c+\delta>n \vee(\neg$ lefto $\wedge \neg$ open $\left.\wedge c+\delta \geq n)\right)$.

\section{Soundness and Completeness of the Encodings}

The encoding just given is sound and complete in the sense defined by the following lemmas which are proven in the appendix.

Lemma 4: The transition system $\mathcal{S}_{p}$ is a sound encoding for $p$ and $\mathcal{S}_{\neg p}$ is a sound encoding for $\neg p$. If a transition system $\mathcal{S}$ over $A P$ is a sound encoding of $\alpha$ and $\beta$, then the transition system $\mathcal{S}_{\mathbf{O p} \alpha}$ over $A P$ is a sound encoding of Op $\alpha$ for each Op $\in\left\{\mathbf{F}_{\leq 0}^{\mathrm{s}}, \mathbf{F}_{<n}^{\mathrm{s}}, \mathbf{F}_{\leq n}^{\mathrm{s}}, \mathbf{G}_{\leq 0}^{\mathrm{s}}, \mathbf{G}_{<n}^{\mathrm{s}}, \mathbf{G}_{\leq n}^{\mathrm{s}}\right\}$, and $\mathcal{S}_{\alpha \mathbf{O p} \beta}$ is a sound encoding of $\alpha \mathbf{O p} \beta$ for each Op $\in$ $\left\{\wedge, \vee, \mathbf{U}^{\mathrm{s}}, \mathbf{U}_{\geq n}^{\mathrm{s}}, \mathbf{U}_{>n}^{\mathrm{s}}, \mathbf{R}^{\mathrm{s}}, \mathbf{R}_{\geq n}^{\mathrm{s}}, \mathbf{R}_{>n}^{\mathrm{s}}\right\}$.

Lemma 5: The transition system $\mathcal{S}_{p}$ is a complete encoding for $p, \mathcal{S}_{\neg p}$ is a complete encoding for $\neg p$. If a transition system $\mathcal{S}$ over $A P$ is a complete encoding of $\alpha$ and $\beta$, then the transition system $\mathcal{S}_{\mathbf{O p} \alpha}$ over $A P$ is a complete encoding of Op $\alpha$ for each $\mathbf{O p} \in\left\{\mathbf{F}_{\leq 0}^{\mathrm{s}}, \mathbf{F}_{<n}^{\mathrm{s}}, \mathbf{F}_{\leq n}^{\mathrm{s}}, \mathbf{G}_{\leq 0}^{\mathrm{s}}, \mathbf{G}_{<n}^{\mathrm{s}}, \mathbf{G}_{\leq n}^{\mathrm{s}}\right\}$, and $\mathcal{S}_{\alpha \mathbf{O p} \beta}$ is a complete encoding of $\alpha \mathbf{O p} \beta$ for each $\mathbf{O p} \in\left\{\wedge, \vee, \mathbf{U}^{\mathrm{s}}, \mathbf{U}_{\geq n}^{\mathrm{s}}, \mathbf{U}_{>n}^{\mathrm{s}}, \mathbf{R}^{\mathrm{s}}, \mathbf{R}_{\geq n}^{\mathrm{s}}, \mathbf{R}_{>n}^{\mathrm{s}}\right\}$

\section{BOUnded MOdel CHECKING}

Naturally, one cannot directly handle infinite formula representations capturing infinite runs with SMT solvers. Thus in bounded model checking $(B M C)$ one considers finite representations, i.e. looping, lasso-shaped paths only. We show that, by using region abstraction [1], we can indeed capture all runs that satisfy a MITL $0, \infty$ formula with such finite representations. For this we must assume that the domains of all the non-clock variables in $Z$ are finite.

Assume a transition system $\langle Z, X, \mathcal{I}, \mathcal{I N} \mathcal{V}, \mathcal{T}, \mathcal{F}, \widehat{A P}\rangle$ over a set $A P$ of atomic propositions. For each clock $x \in X$, let $\mathrm{m}_{x}$ be the largest constant $n$ occurring in atoms of forms $x \bowtie n$ and $x+\delta \bowtie n$ in $\mathcal{I}, \mathcal{I N} \mathcal{V}$, and $\mathcal{T}$. Two states, $s$ and $t$ (i.e. valuations over $Z \cup X$ as defined in Sect. IV-A), belong to the same equivalence class called region, denoted by $s \approx t$, if (i) $s(z)=t(z)$ for each non-clock variable $z \in Z$, and (ii) for all clocks $x, y \in X$ 
1) either (a) $\lfloor s(x)\rfloor=\lfloor t(x)\rfloor$ or (b) $s(x)>\mathrm{m}_{x}$ and $t(x)>\mathrm{m}_{x}$;

2) if $s(x) \leq \mathrm{m}_{x}$, then $\operatorname{fract}(s(x))=0$ iff fract $(t(x))=$ 0 , where fract $(i)$ denotes the fractional part of $i$; and

3) if $s(x) \leq \mathrm{m}_{x}$ and $s(y) \leq \mathrm{m}_{y}$, then fract $(s(x)) \leq$ fract $(s(y))$ iff fract $(t(x)) \leq \operatorname{fract}(t(y))$.

Next, we will apply the bisimulation property of regions introduced in [1] to transition systems.

Lemma 6: Assume two states, $s$ and $t$, such that $s \approx t$.

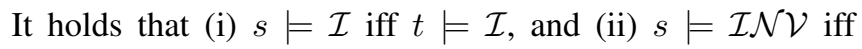
$t=\mathcal{I N} \mathcal{V}$. Furthermore, if there is a $\delta_{s} \in \mathbb{R}_{\geq 0}$ and a state $s^{\prime}$ such that $s \cup\left\{\delta \mapsto \delta_{s}\right\} \cup\left\{y^{\prime} \mapsto s^{\prime}(y) \mid y \in X \cup Z\right\} \models$ $\mathcal{T}$, then there is a $\delta_{t} \in \mathbb{R}_{\geq 0}$ and a state $t^{\prime}$ such that $t \cup\left\{\delta \mapsto \delta_{t}\right\} \cup\left\{y^{\prime} \mapsto t^{\prime}(y) \mid y \in X \cup Z\right\} \mid=\mathcal{T}$ and $s^{\prime} \approx t^{\prime}$. Lemma 6 is proven in the appendix.

When the domains of the non-clock variables are finite, as we have assumed, the set of equivalence classes induced by $\approx$ is finite, too. In this case we can prove, in a similar fashion as the corresponding lemma in [12], that all runs of a transition system also have corresponding runs whose projections on the equivalences classes induced by $\approx$ are lasso-shaped looping runs:

Lemma 7: Let $V a$ al be the set of all valuations over $Z$ and $R e g$ the set of clock regions. If the transition system $\mathcal{S}$ has an arbitrary infinite run starting in some state $s_{0}$, then it also has a run run $\tau=s_{0} \stackrel{\delta_{0}}{\longrightarrow} s_{1} \stackrel{\delta_{1}}{\longrightarrow} s_{2} \stackrel{\delta_{2}}{\longrightarrow} \ldots$ such that for some $i, k \in \mathbb{N}$ with $0 \leq i \leq k \leq(|X|+|\mathcal{F}|+2) \cdot \mid$ Val $|\cdot| \operatorname{Reg} \mid$ and for every $j$ with $j \geq i$ we have $s_{j} \approx s_{j+k-i+1}$.

Intuitively, Lemma 7 states that if $\mathcal{S}$ has a run starting in a given state, then $\mathcal{S}$ has a run starting in the same state that begins to loop through the same regions after a finite prefix. E.g., if $i=7$ and $k=10$, then $s_{7} \approx$ $s_{11} \approx s_{15} \approx s_{19} \ldots$ and $s_{8} \approx s_{12} \approx s_{16} \approx s_{20} \ldots$. In particular, Lemma 7 implies that if we are interested in whether $\mathcal{S}$ has any run at all, it is sufficient to search for runs that are lasso-shaped under the region abstraction. Such runs can be captured with finite bounded model checking encodings. Given a formula $\psi$ over $Z \cup X \cup\{\delta\} \cup Z^{\prime} \cup X^{\prime}$ and an index $i \in \mathbb{N}$, let $\psi^{[i]}$ be the the formula over $\left\{y^{[i]} \mid y \in Z \cup X \cup\{\delta\}\right\} \cup\left\{y^{[i+1]} \mid y \in Z \cup X\right\}$ obtained by replacing each variable $y \in Z \cup X \cup\{\delta\}$ with the variable $y^{[i]}$ and each $y^{\prime} \in Z^{\prime} \cup X^{\prime}$ with the variable $y^{[i+1]}$. E.g., $\left(\left(x^{\prime}=x+\delta\right) \wedge \neg p\right)^{[3]}$ is $\left.\left(x^{[4]}=x^{[3]}+\delta^{[3]}\right) \wedge \neg p^{[3]}\right)$. Now the bounded model checking encoding for bound $k$ is:

$$
\begin{aligned}
\|\mathcal{S}, k\|:= & \delta^{[0]}=0 \wedge \mathcal{I}^{[0]} \wedge \bigwedge_{0 \leq j \leq k} \mathcal{I N} \mathcal{V}^{[j]} \wedge \\
& \bigwedge_{0 \leq j<k} \mathcal{T}^{[j]} \wedge \bigwedge_{1 \leq j \leq k}\left(\text { loop }^{[j]} \Rightarrow E_{j, k}\right) \wedge \\
& \bigwedge_{0 \leq j<k}\left(\delta^{[j]}>0 \Rightarrow \delta^{[j+1]}=0\right) \wedge \\
& \text { Fair }_{k} \wedge \text { NonZeno }_{k} \wedge \bigvee_{1 \leq j \leq k} \text { loop }^{[j]}
\end{aligned}
$$

where (i) $E_{j, k}$ is a formula evaluating to true if state $j-1$ and state $k$ (i.e. the valuations of the variables with superscripts $j-1$ and $k$, respectively) are in the same region (see [12] for different ways to implement this), and (ii) $\mathrm{Fair}_{k}$ and

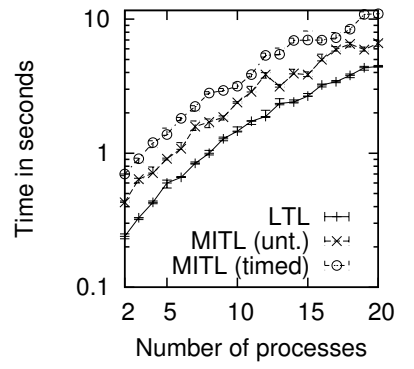

(a) Non-holding property

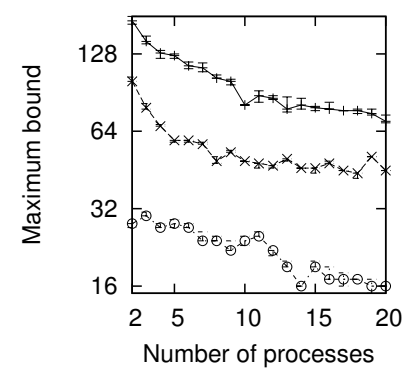

(b) Holding property
Figure 5. Experimental results

NonZeno $_{k}$ are constraints forcing that the fairness formulas are holding in the loop and that sufficiently much time passes in the loop to unroll it to a non-zeno run (again, see [12]). Intuitively, the conjuncts of $\|\mathcal{S}, k\|$ encode the following: (a) the first interval is a singleton and satisfies the initial constraint, (b) all intervals satisfy the invariant and all pairs of successive states the transition relation, (c) if some $l_{o o p}{ }^{[j]}$ holds then state $j-1$ and state $k$ are in the same region, (d) there are no two successive open intervals, (e) the fairness formulas are satisfied within the looping part of the trace, (f) the trace is non-zeno and (g) at least one $l o o p^{[j]}$ is true, meaning that the trace is "looping under region abstraction".

Now, if we wish to find out whether a transition system $\mathcal{S}$ has a run corresponding to a trace $\sigma$ such that $\sigma=\phi$ for a $\mathrm{MITL}_{0, \infty}$ formula $\phi$, we can check whether $\left\|\mathcal{S}_{\phi}, k\right\| \wedge$ $\| \phi \rrbracket^{[0]}$ is satisfiable for some $0<k \leq(|X|+|\mathcal{F}|+2)$. $|V a l| \cdot|R e g|$. This upper bound is very large and, in practice, much lower bounds are often used (and sufficient for finding traces). Then, however, the possibility remains that a trace exists despite none being found with the bound used.

\section{EXPERIMENTAL EVALUATION}

We have studied the feasibility of the BMC encoding developed in this paper experimentally. We have devised a straightforward implementation of the approach following the encoding scheme given in Sect. IV and V. With experiments on a class of models we (i) show that it is possible to develop relatively efficient implementations of the approach, (ii) demonstrate that the approach scales reasonably and (iii) are able to estimate the cost of timing by comparing the verification of properties using timed operators both to verifying $\mathrm{MITL}_{0, \infty}$ properties that do not use timing constraints and region-based LTL BMC [12], [13].

As a model for the experimentation we used the Fischer mutual exclusion protocol with two to 20 agents. This protocol is commonly used for the evaluation of timed verification approaches. The encoding used for the experiments is based on a model that comes with the model checker Uppaal [2] which also uses super-dense time. We checked one property 
that holds ("requesting state leads to waiting state eventually") and one that does not ("there is no trace visiting the critical section and the non-critical section infinitely often"). ${ }^{3}$ Each property was checked in three variants: as an LTL property using the approach from [12], as the corresponding MITL property (only untimed operators) and with timing constraints added. Both MITL BMC and LTL $\mathrm{BMC}$ were used in an incremental fashion, i.e. bounds are increased starting with bound one until a counter-example is found and constraints are shared by successive SMT solver calls where possible. All experiments were run under Linux on Intel Xeon X5650 CPUs limiting memory to 4 GB and CPU time to 20 minutes. As an SMT solver, Yices [14] version 1.0.37 was used. All plots report minimum, maximum and median over 11 executions. The implementation and the benchmark used are available on the first author's website.

Figure 5a shows the time needed for finding a counterexample to the non-holding property. No timeouts were encountered, even when using the timed MITL properties. Figures $5 \mathrm{~b}$ shows the maximum bound reached within 20 minutes when checking the holding property. The bounds reached for the timed property are significantly lower than the bounds reached for the LTL property with the untimed MITL BMC bounds lying between. While there is both a cost for using the MITL framework for an untimed property and an additional cost for adding timing constraints, checking timed constraints using MITL BMC is certainly feasible. The performance could be further improved using wellknown optimization techniques e.g. by adding the possibility for finite counter-examples [11], a technique used in the LTL BMC implementation used for the experiments. When verifying properties without timing constraints, using LTL $\mathrm{BMC}$, however, is advisable not only because of the better performance but also because a lower bound is needed to find a trace as open intervals are irrelevant for LTL formulas.

\section{CONCLUSIONS}

In this paper, we extend the linear time logic $\mathrm{MITL}_{0, \infty}$ to super-dense time semantics. We devise a method to encode both a timed automaton and a $\operatorname{MITL}_{0, \infty}$ formula as a symbolic transition system. The encoding provides a foundation for different kinds of fully symbolic verification methods. Soundness and completeness of the encoding are proven in the appendix. Furthermore, we demonstrate how the encoding can be employed for bounded model checking (BMC) using the well-known region abstraction. We have implemented the approach. An experimental evaluation of the BMC approach indicated that a reasonably efficient implementation is feasible.

\section{ACKNOWLEDGEMENTS}

This work has been financially supported by the Academy of Finland under project 128050 and under the Finnish

\footnotetext{
${ }^{3}$ Here, we search for counter-examples, i.e. encode $\neg \phi$ instead of $\phi$.
}

Centre of Excellence in Computational Inference (COIN).

\section{REFERENCES}

[1] R. Alur and D. L. Dill, "A theory of timed automata," Theoretical Computer Science, vol. 126, no. 2, pp. 183-235, 1994.

[2] G. Behrmann, A. David, and K. G. Larsen, "A tutorial on UPPAAL," in Proc. FM-RT 2004, ser. LNCS, vol. 3185. Springer, September 2004, pp. 200-236.

[3] R. Alur, T. Feder, and T. A. Henzinger, "The benefits of relaxing punctuality," Journal of the ACM, vol. 43, no. 1, pp. 116-146, 1996.

[4] O. Maler, D. Nickovic, and A. Pnueli, "From MITL to timed automata," in FORMATS, ser. LNCS, vol. 4202. Springer, 2006, pp. 274-289.

[5] R. Alur, "Timed automata," in Proc. CAV 1999, ser. LNCS, vol. 1633. Springer, 1999, pp. 8-22.

[6] J. Bengtsson and W. Yi, "Timed automata: Semantics, algorithms and tools," in Lectures on Concurrency and Petri Nets, ser. LNCS, vol. 3098. Springer, 2004, pp. 87-124.

[7] M. Sorea, "Bounded model checking for timed automata," Elect. Notes Theor. Comp. Sci., vol. 68, no. 5, pp. 116-134, 2002.

[8] G. Audemard, A. Cimatti, A. Kornilowicz, and R. Sebastiani, "Bounded model checking for timed systems," in Proc. FORTE 2002, ser. LNCS, vol. 2529. Springer, 2002, pp. 243-259.

[9] R. Kindermann, T. Junttila, and I. Niemelä, "Modeling for symbolic analysis of safety instrumented systems with clocks," in Proc. ACSD 2011. IEEE, 2011, pp. 185-194.

[10] C. Barrett, R. Sebastiani, S. A. Seshia, and C. Tinelli, "Satisfiability modulo theories," in Handbook of Satisfiability. IOS Press, 2009, pp. 825-885.

[11] A. Biere, K. Heljanko, T. Junttila, T. Latvala, and V. Schuppan, "Linear encodings of bounded LTL model checking," Logical Methods in Computer Science, vol. 2, no. 5:5, pp. 1-64, 2006.

[12] R. Kindermann, T. Junttila, and I. Niemelä, "Beyond lassos: Complete SMT-based bounded model checking for timed automata," in Proc. FORTE 2012, ser. LNCS, vol. 7273. Springer, 2012, pp. 84-100.

[13] A. Biere, A. Cimatti, E. M. Clarke, and Y. Zhu, "Symbolic model checking without BDDs," in Proc. TACAS 1999, ser. LNCS, vol. 1579. Springer, 1999, pp. 193-207.

[14] B. Dutertre and L. M. de Moura, "A fast linear-arithmetic solver for DPLL(T)," in Proc. CAV 2006, ser. LNCS, vol. 4144. Springer, 2006, pp. 81-94. 


\section{APPENDIX}

\section{A. Duality of until and release operators}

Lemma 8: For any trace $\sigma=\left\langle I_{0}, v_{0}\right\rangle,\left\langle I_{1}, v_{1}\right\rangle, \ldots$ over $A P$, MITL $_{0, \infty}$ formulas $\phi$ and $\psi$ over $A P, i \in \mathbb{N}, t \in I_{i}$ it holds that $\sigma^{(i, t)}=\left(\phi \mathbf{U}_{\bowtie n}^{\mathrm{s}} \psi\right)$ iff $\sigma^{(i, t)}=\neg\left(\neg \phi \mathbf{R}_{\bowtie n}^{\mathrm{s}} \neg \psi\right)$

Proof: $\sigma^{(i, t)} \models \neg\left(\neg \phi \mathbf{R}_{\bowtie n}^{\mathrm{s}} \neg \psi\right)$ if and only if (by definition)

$\neg \forall\left(i^{\prime}, t^{\prime}\right) \in T_{+}(\sigma,(i, t)):\left(\left(t^{\prime}-t \bowtie n\right) \wedge \neg\left(\sigma^{\left(i^{\prime}, t^{\prime}\right)}=\right.\right.$ $\neg \psi)) \Rightarrow\left(\exists\left(i^{\prime \prime}, t^{\prime \prime}\right) \in T_{+}(\sigma,(i, t)):\left(i^{\prime \prime}, t^{\prime \prime}\right) \prec\left(i^{\prime}, t^{\prime}\right) \wedge\right.$ $\left.\left(\sigma^{\left(i^{\prime \prime}, t^{\prime \prime}\right)} \models \neg \phi\right)\right)$

if and only if (pushing negations inside)

$\exists\left(i^{\prime}, t^{\prime}\right) \in T_{+}(\sigma,(i, t)):\left(\left(t^{\prime}-t \bowtie n\right) \wedge \neg\left(\sigma^{\left(i^{\prime}, t^{\prime}\right)} \models\right.\right.$ $\neg \psi)) \wedge\left(\forall\left(i^{\prime \prime}, t^{\prime \prime}\right) \in T_{+}(\sigma,(i, t)):\left(i^{\prime \prime}, t^{\prime \prime}\right) \prec\left(i^{\prime}, t^{\prime}\right) \Rightarrow\right.$ $\left.\neg\left(\sigma^{\left(i^{\prime \prime}, t^{\prime \prime}\right)} \models \neg \phi\right)\right)$

if and only if (replacing $\left.\neg\left(\sigma^{\left(i^{\prime}, t^{\prime}\right)} \models \neg \cdot\right)\right)$ by $\left(\sigma^{\left(i^{\prime}, t^{\prime}\right)} \models \cdot\right)$ ) $\left.\exists\left(i^{\prime}, t^{\prime}\right) \in T_{+}(\sigma,(i, t)):\left(t^{\prime}-t \bowtie n\right) \wedge\left(\sigma^{\left(i^{\prime}, t^{\prime}\right)} \models \psi\right)\right) \wedge$ $\left(\forall\left(i^{\prime \prime}, t^{\prime \prime}\right) \in T_{+}(\sigma,(i, t)):\left(i^{\prime \prime}, t^{\prime \prime}\right) \prec\left(i^{\prime}, t^{\prime}\right) \Rightarrow\left(\sigma^{\left(i^{\prime \prime}, t^{\prime \prime}\right)} \models\right.\right.$ $\phi))$

if and only if (by definition) $\sigma^{(i, t)} \models\left(\phi \mathbf{U}_{\bowtie n}^{\mathrm{s}} \psi\right)$.

Lemma 9: For any trace $\sigma=\left\langle I_{0}, v_{0}\right\rangle,\left\langle I_{1}, v_{1}\right\rangle, \ldots$ over $A P$, MITL $_{0, \infty}$ formulas $\phi$ and $\psi$ over $A P, i \in \mathbb{N}, t \in I_{i}$ it holds that $\sigma^{(i, t)}=\left(\phi \mathbf{R}_{\bowtie n}^{\mathrm{s}} \psi\right)$ iff $\sigma^{(i, t)}=\neg\left(\neg \phi \mathbf{U}_{\bowtie n}^{\mathrm{s}} \neg \psi\right)$

Proof: $\sigma^{(i, t)} \models \neg\left(\neg \phi \mathbf{U}_{\bowtie n}^{\mathrm{s}} \neg \psi\right)$ iff (by Lemma 8) $\sigma^{(i, t)} \models \neg\left(\neg\left(\neg \neg \phi \quad \mathbf{R}_{\bowtie n}^{\mathrm{s}} \neg \neg \psi\right)\right)$ iff (double negations) $\sigma^{(i, t)} \mid=\left(\phi \mathbf{R}_{\bowtie n}^{\mathrm{s}} \psi\right)$.

\section{B. Proof of Lemma 1}

Lemma 1: If a trace $\sigma$ is fine for $\phi$ and $\psi, i \in \mathbb{N}, t, u \in I_{i}$, $\triangleleft \in\{<, \leq\}$, and $\triangleright \in\{\geq,>\}$, then

- if $\sigma^{(i, t)} \models \phi \mathbf{U}_{\triangleleft n}^{\mathrm{s}} \psi$ and $u \geq t$, then $\sigma^{(i, u)} \models \phi \mathbf{U}_{\triangleleft n}^{\mathrm{s}} \psi$;

- if $\sigma^{(i, t)} \models \phi \mathbf{U}_{\triangleright n}^{\mathrm{s}} \psi$ and $u \leq t$, then $\sigma^{(i, u)}=\phi \mathbf{U}_{\triangleright n}^{\mathrm{s}} \psi$;

- if $\sigma^{(i, t)} \models \phi \mathbf{R}_{\triangleleft n}^{\mathrm{s}} \psi$ and $u \leq t$, then $\sigma^{(i, u)} \models \phi \mathbf{R}_{\triangleleft n}^{\mathbf{s}} \psi$;

- if $\sigma^{(i, t)}=\phi \mathbf{R}_{\triangleright n}^{\mathrm{s}} \psi$ and $u \geq t$, then $\sigma^{(i, u)}=\phi \mathbf{R}_{\triangleright n}^{\mathrm{s}} \psi$.

Proof: If $I_{i}$ is a singleton, then the lemma holds trivially. Thus, assume that $I_{i}$ is an open interval. We have the following four cases.

- Assume that $\sigma^{(i, t)} \models \phi \mathbf{U}_{\triangleleft n}^{\mathrm{s}} \psi$. Thus there exists a $\left(i^{\prime}, t^{\prime}\right) \in T_{+}(\sigma,(i, t))$ such that $\left(t^{\prime}-t \triangleleft n\right) \wedge\left(\sigma^{\left(i^{\prime}, t^{\prime}\right)} \models\right.$ $\psi) \wedge\left(\forall\left(i^{\prime \prime}, t^{\prime \prime}\right) \in T_{+}(\sigma,(i, t)),\left(i^{\prime \prime}, t^{\prime \prime}\right) \prec\left(i^{\prime}, t^{\prime}\right) \Rightarrow\right.$ $\left.\left(\sigma^{\left(i^{\prime \prime}, t^{\prime \prime}\right)} \models \phi\right)\right)$. Let $u \geq t$ with $u \in I_{i}$. Now $T_{+}(\sigma,(i, u)) \subseteq T_{+}(\sigma,(i, t))$.

If $i^{\prime}>i$ or $i^{\prime}=i \wedge u<t^{\prime}$, then $\left(t^{\prime}-u \triangleleft n\right) \wedge\left(\sigma^{\left(i^{\prime}, t^{\prime}\right)}=\right.$ $\psi) \wedge\left(\forall\left(i^{\prime \prime}, t^{\prime \prime}\right) \in T_{+}(\sigma,(i, u)),\left(i^{\prime \prime}, t^{\prime \prime}\right) \prec\left(i^{\prime}, t^{\prime}\right) \Rightarrow\right.$ $\left.\left(\sigma^{\left(i^{\prime \prime}, t^{\prime \prime}\right)} \models \phi\right)\right)$, implying $\sigma^{(i, u)} \models \phi \mathbf{U}_{\triangleleft n}^{\mathrm{s}} \psi$ irrespective whether $\sigma$ is fine for $\phi$ and $\psi$ or not.

If $i^{\prime}=i$ and $u \geq t^{\prime}$, then there is a $u^{\prime}>u$ with $u^{\prime} \in I_{i}$ and $u^{\prime}-u \triangleleft n$ as $I_{i}$ is an open interval. As $\sigma$ is fine for $\psi$ and $\sigma^{\left(i, t^{\prime}\right)} \models \psi$, it holds that $\sigma^{\left(i, u^{\prime}\right)}=\psi$ as well. As $\forall\left(i, t^{\prime \prime}\right) \in T_{+}(\sigma,(i, t)),\left(i, t^{\prime \prime}\right) \prec\left(i, t^{\prime}\right) \Rightarrow\left(\sigma^{\left(i, t^{\prime \prime}\right)} \models\right.$ $\phi)$, there is at least one $t<t^{\prime \prime}<t^{\prime}$, and $\sigma$ is fine for $\phi$, we have $\forall\left(i, u^{\prime \prime}\right) \in T_{+}(\sigma,(i, u)),\left(i, u^{\prime \prime}\right) \prec\left(i, u^{\prime}\right) \Rightarrow$ $\left(\sigma^{\left(i, u^{\prime \prime}\right)} \models \phi\right)$. Therefore, $\sigma^{(i, u)} \models \phi \mathbf{U}_{\triangleleft n}^{\mathrm{s}} \psi$.
- Assume that $\sigma^{(i, t)} \models \phi \mathbf{U}_{\triangleright n}^{\mathrm{s}} \psi$. Thus there exists a $\left(i^{\prime}, t^{\prime}\right) \in T_{+}(\sigma,(i, t))$ such that $\left(t^{\prime}-t \triangleright n\right) \wedge\left(\sigma^{\left(i^{\prime}, t^{\prime}\right)} \equiv\right.$ $\psi) \wedge\left(\forall\left(i^{\prime \prime}, t^{\prime \prime}\right) \in T_{+}(\sigma,(i, t)),\left(i^{\prime \prime}, t^{\prime \prime}\right) \prec\left(i^{\prime}, t^{\prime}\right) \Rightarrow\right.$ $\left.\left(\sigma^{\left(i^{\prime \prime}, t^{\prime \prime}\right)}=\phi\right)\right)$. Let $u \leq t$ with $u \in I_{i}$. Thus $t^{\prime}-u \triangleright$ $n$. Because (i) $\forall\left(i^{\prime \prime}, t^{\prime \prime}\right) \in T_{+}(\sigma,(i, t)),\left(i^{\prime \prime}, t^{\prime \prime}\right) \prec$ $\left(i^{\prime}, t^{\prime}\right) \Rightarrow\left(\sigma^{\left(i^{\prime \prime}, t^{\prime \prime}\right)} \models \phi\right)$, (ii) there is at least one $t<t^{\prime \prime}<t^{\prime}$ with $t^{\prime \prime} \in I_{i}$ as $I_{i}$ is open, and (iii) $\sigma$ is fine for $\phi$, we have $\forall\left(i^{\prime \prime}, t^{\prime \prime}\right) \in T_{+}(\sigma,(i, u)),\left(i^{\prime \prime}, t^{\prime \prime}\right) \prec$ $\left(i^{\prime}, t^{\prime}\right) \Rightarrow\left(\sigma^{\left(i^{\prime \prime}, t^{\prime \prime}\right)} \models \phi\right)$. Therefore, $\sigma^{(i, u)}=\phi \mathbf{U}_{\triangleleft n}^{\mathrm{s}} \psi$.

- Assume that $\sigma^{(i, t)}=\phi \mathbf{R}_{\triangleleft n}^{\mathrm{s}} \psi$. Thus $\forall\left(i^{\prime}, t^{\prime}\right) \in$ $T_{+}(\sigma,(i, t)),\left(\left(t^{\prime}-t \triangleleft n\right) \wedge \neg\left(\sigma^{\left(i^{\prime}, t^{\prime}\right)} \models \psi\right)\right) \Rightarrow$ $\left(\exists\left(i^{\prime \prime}, t^{\prime \prime}\right) \in T_{+}(\sigma,(i, t)),\left(i^{\prime \prime}, t^{\prime \prime}\right) \prec\left(i^{\prime}, t^{\prime}\right) \wedge\right.$ $\left.\left(\sigma^{\left(i^{\prime \prime}, t^{\prime \prime}\right)} \models \phi\right)\right)$. Let $u \leq t$ with $u \in I_{i}$.

Suppose that $\left(u^{\prime}-t^{\prime} \triangleleft n\right) \wedge \neg\left(\sigma^{\left(j^{\prime}, u^{\prime}\right)} \models \psi\right)$ for some $\left(j, u^{\prime}\right) \in T_{+}(\sigma,(i, u))$. If $(i, t) \prec\left(j^{\prime}, u^{\prime}\right)$, then $\exists\left(i^{\prime \prime}, t^{\prime \prime}\right) \in T_{+}(\sigma,(i, t)),\left(i^{\prime \prime}, t^{\prime \prime}\right) \prec\left(j^{\prime}, u^{\prime}\right) \wedge$ $\left(\sigma^{\left(i^{\prime \prime}, t^{\prime \prime}\right)} \models \phi\right)$. On the other hand, if $\left(j^{\prime}, u^{\prime}\right)=(i, t)$ or $\left(j^{\prime}, u^{\prime}\right) \prec(i, t)$, then $j^{\prime}=i, \neg\left(\sigma^{(i, v)}=\psi\right)$ for all $v \in I_{i}$ as $\sigma$ is fine for $\psi$, there is a $\left(i, t^{\prime \prime}\right) \in$ $T_{+}(\sigma,(i, t)),(i, t) \prec\left(i, t^{\prime \prime}\right) \wedge\left(\sigma^{\left(i, t^{\prime \prime}\right)} \models \phi\right)$ as $I_{i}$ is open, $\sigma^{(i, v)}=\phi$ for all $v \in I_{i}$ as $\sigma$ is fine for $\phi$, and $\sigma^{(i, u)}=\phi \mathbf{R}_{\triangleleft n}^{\mathrm{s}} \psi$.

- Assume that $\sigma^{(i, t)} \models \phi \mathbf{R}_{\triangleright n}^{\mathrm{s}} \psi$. Thus $\forall\left(i^{\prime}, t^{\prime}\right) \in$ $T_{+}(\sigma,(i, t)),\left(\left(t^{\prime}-t \triangleright n\right) \wedge \neg\left(\sigma^{\left(i^{\prime}, t^{\prime}\right)} \models \psi\right)\right) \Rightarrow$ $\left(\exists\left(i^{\prime \prime}, t^{\prime \prime}\right) \in T_{+}(\sigma,(i, t)),\left(i^{\prime \prime}, t^{\prime \prime}\right) \prec\left(i^{\prime}, t^{\prime}\right) \wedge\right.$ $\left.\left(\sigma^{\left(i^{\prime \prime}, t^{\prime \prime}\right)} \models \phi\right)\right)$. Let $u \geq t$ with $u \in I_{i}$. Suppose that $\left(u^{\prime}-u \triangleright n\right) \wedge \neg\left(\sigma^{\left(j^{\prime}, u^{\prime}\right)} \models \psi\right)$ for some $\left(j^{\prime}, u^{\prime}\right) \in$ $T_{+}(\sigma,(i, u))$. As $u^{\prime}-t \triangleright n$, there exists a $\left(i^{\prime \prime}, t^{\prime \prime}\right) \in$ $T_{+}(\sigma,(i, t))$ such that $\left(i^{\prime \prime}, t^{\prime \prime}\right) \prec\left(i^{\prime}, t^{\prime}\right) \wedge\left(\sigma^{\left(i^{\prime \prime}, t^{\prime \prime}\right)}=\right.$ $\phi))$. If $\left(j^{\prime}, u^{\prime}\right) \prec\left(i^{\prime \prime}, t^{\prime \prime}\right)$, we are done. On the other hand, if $\left(i^{\prime \prime}, t^{\prime \prime}\right)=\left(j^{\prime}, u^{\prime}\right)$ or $\left(i^{\prime \prime}, t^{\prime \prime}\right) \prec\left(j^{\prime}, u^{\prime}\right)$, then $\sigma^{(i, v)}=\phi$ for all $v \in I_{i}$ as $\sigma$ is fine for $\phi$, there exists a $\left(i, j^{\prime \prime}\right)$ such that $(i, j) \prec\left(i, j^{\prime \prime}\right) \prec\left(j^{\prime}, u^{\prime}\right)$ as $I_{i}$ is open, and thus $\sigma^{(i, u)}=\phi \mathbf{R}_{\triangleright n}^{\mathrm{s}} \psi$.

\section{Proof of Lemma 2}

Lemma 2: Let $\phi$ be a $\mathrm{MITL}_{0, \infty}$ formula and $\sigma$ a trace. There is a refinement $\sigma^{\prime}$ of $\sigma$ that is $\phi$-fine. Such a refinement can be obtained by splitting each open interval in $\sigma$ into at most $2^{K}$ new open intervals and $2^{K}-1$ singletons, where $K$ is the number of timed until and release operators in $\phi$.

Proof: Let $\left[\phi_{1}, \ldots, \phi_{n}\right]$ be a list containing all the subformulas of $\phi$ so that the sub-formulas of a sub-formula $\phi_{i}$ are listed before $\phi_{i}$. Thus $\phi_{1}$ is an atomic proposition and $\phi_{n}=\phi$.

We now construct a trace $\sigma_{i}$ for each $1 \leq i \leq n$ such that $\sigma_{i}$ is fine for all sub-formulas $\phi_{j}$ with $1 \leq j \leq i$.

If $\phi_{i}$ is an atomic proposition or of forms $\neg \phi_{j}, \phi_{j} \wedge \phi_{k}$, or $\phi_{j} \vee \phi_{k}$ with $j, k<i$, then $\sigma_{i}=\sigma_{i-1}$ is fine for $\phi_{i}$ as well.

If $\phi_{i}$ is an until or release formula of forms $\phi_{j} \mathbf{U}_{\bowtie n}^{\mathrm{s}} \phi_{k}$ or $\phi_{j} \mathbf{R}_{\bowtie n}^{\mathrm{s}} \phi_{k}$, then by (i) recalling that $\sigma_{i-1}$ is fine for $\phi_{j}$ 
and $\phi_{k}$ (ii) applying Lemma 1 , we obtain a $\phi_{i}$-fine trace $\sigma_{i}$ by splitting each open interval in $\sigma_{i-1}$ into at most two new open intervals and one singleton interval.

\section{Proof of Lemma 3}

Lemma 3: $\sigma^{(i, t)}=\phi \mathbf{U}_{\triangleleft n}^{\mathrm{s}} \psi$ iff $\sigma^{(i, t)}=\left(\mathbf{F}_{\triangleleft n}^{\mathrm{s}} \psi\right) \wedge\left(\phi \mathbf{U}^{\mathrm{s}}\right.$ $\psi$ ) for all $i \in \mathbb{N}, t \in I_{i}, \triangleleft \in\{<, \leq\}$, and $n \in \mathbb{N}$.

Proof: Recall that $\sigma^{(i, t)} \models\left(\phi \mathbf{U}_{\bowtie n}^{\mathrm{s}} \psi\right)$ iff $\exists\left(i^{\prime}, t^{\prime}\right) \in$ $T_{+}(\sigma,(i, t)):\left(t^{\prime}-t \bowtie n\right) \wedge\left(\sigma^{\left(i^{\prime}, t^{\prime}\right)}=\psi\right) \wedge\left(\forall\left(i^{\prime \prime}, t^{\prime \prime}\right) \in\right.$ $\left.T_{+}(\sigma,(i, t)):\left(i^{\prime \prime}, t^{\prime \prime}\right) \prec\left(i^{\prime}, t^{\prime}\right) \Rightarrow\left(\sigma^{\left(i^{\prime \prime}, t^{\prime \prime}\right)} \models \phi\right)\right)$.

- The “ $\Rightarrow$ " part.

As is easy to see from the semantics, $\sigma^{(i, t)}=\left(\phi \mathbf{U}_{\bowtie n}^{\mathrm{s}}\right.$ $\psi$ ) implies both (i) $\sigma^{(i, t)} \models\left(\phi \mathbf{U}^{\mathrm{s}} \psi\right)$ and (ii) $\sigma^{(i, t)} \models$ (true $\mathbf{U}_{\bowtie n}^{\mathrm{s}} \psi$ ) corresponding to $\sigma^{(i, t)} \models \mathbf{F}_{\bowtie n}^{\mathrm{s}} \psi$.

- The " $\Leftarrow$ " part.

By the semantics, if $\sigma^{(i, t)} \models\left(\phi \mathbf{U}^{\mathrm{s}} \psi\right)$ we can pick a $\left(i^{\prime}, t^{\prime}\right) \in T_{+}(\sigma,(i, t))$ such that $\left(t^{\prime}-t \geq 0\right) \wedge$ $\left(\sigma^{\left(i^{\prime}, t^{\prime}\right)}=\psi\right) \wedge\left(\forall\left(i^{\prime \prime}, t^{\prime \prime}\right) \in T_{+}(\sigma,(i, t)):\left(i^{\prime \prime}, t^{\prime \prime}\right) \prec\right.$ $\left.\left(i^{\prime}, t^{\prime}\right) \Rightarrow\left(\sigma^{\left(i^{\prime \prime}, t^{\prime \prime}\right)}=\phi\right)\right)$. We have two cases now:

- If $t^{\prime}-t \triangleleft n$, then we immediately have $\sigma^{(i, t)}=$ $\left(\phi \mathbf{U}_{\triangleleft n}^{\mathrm{s}} \psi\right)$.

- Otherwise, $\sigma^{(i, t)} \models \mathbf{F}_{\triangleleft n}^{\mathrm{s}} \psi$ allows us to pick $\left(j^{\prime}, u^{\prime}\right) \in T_{+}(\sigma,(i, t))$ such that $\left(u^{\prime}-t \triangleleft n\right) \wedge$ $\left(\sigma^{\left(j^{\prime}, u^{\prime}\right)}=\psi\right)$. As $u^{\prime}-t \triangleleft n$, we know that $\left(j^{\prime}, u^{\prime}\right) \prec\left(i^{\prime}, t^{\prime}\right)$, which in turn implies that $\forall\left(i^{\prime \prime}, t^{\prime \prime}\right) \in T_{+}(\sigma,(i, t)):\left(i^{\prime \prime}, t^{\prime \prime}\right) \prec\left(j^{\prime}, u^{\prime}\right) \Rightarrow$ $\left(\sigma^{\left(i^{\prime \prime}, t^{\prime \prime}\right)} \models \phi\right)$. Thus we obtain $\sigma^{(i, t)} \models\left(\phi \mathbf{U}_{\triangleleft n}^{\mathrm{s}}\right.$ $\psi)$.

\section{E. Soundness proofs}

Lemma 4: The transition system $\mathcal{S}_{p}$ is a sound encoding for $p$ and $\mathcal{S}_{\neg p}$ is a sound encoding for $\neg p$. If a transition system $\mathcal{S}$ over $A P$ is a sound encoding of $\alpha$ and $\beta$, then the transition system $\mathcal{S}_{\mathbf{O p} \alpha}$ over $A P$ is a sound encoding of Op $\alpha$ for each Op $\in\left\{\mathbf{F}_{\leq 0}^{\mathrm{s}}, \mathbf{F}_{<n}^{\mathrm{s}}, \mathbf{F}_{\leq n}^{\mathrm{s}}, \mathbf{G}_{\leq 0}^{\mathrm{s}}, \mathbf{G}_{<n}^{\mathrm{s}}, \mathbf{G}_{\leq n}^{\mathrm{s}}\right\}$, and $\mathcal{S}_{\alpha \mathbf{O p} \beta}$ is a sound encoding of $\alpha \mathbf{O p} \beta$ for each $\mathbf{O p} \in$ $\left\{\wedge, \vee, \mathbf{U}^{\mathrm{s}}, \mathbf{U}_{\geq n}^{\mathrm{s}}, \mathbf{U}_{>n}^{\mathrm{s}}, \mathbf{R}^{\mathrm{s}}, \mathbf{R}_{\geq n}^{\mathrm{s}}, \mathbf{R}_{>n}^{\mathrm{s}}\right\}$

Recall, that we call an encoding $\mathcal{S}_{\phi}$ is sound if the following are satisfied:

- all the traces of $\mathcal{S}$ (i.e, projections of runs to the atomic propositions) are preserved: $\operatorname{traces}\left(\mathcal{S}_{\phi}\right)=\operatorname{traces}(\mathcal{S})$

- when $\phi$ is holds in a state, then it holds in the corresponding interval: for each run $\tau=s_{0} s_{1} \ldots$ of $\mathcal{S}_{\phi}$ with $\operatorname{trace}(\tau)=\sigma=\left\langle I_{0}, v_{0}\right\rangle\left\langle I_{1}, v_{1}\right\rangle \ldots$, and each $\left.i \in \mathbb{N}, s_{i}(\| \phi] \|\right)=$ true implies $\forall t \in I_{i}: \sigma^{(i, t)} \models \phi$.

We will now prove Lemma 4 separately for each operator. Note, that as we assumed $\mathcal{S}$ to be sound for $\alpha$ and $\beta$, we know that any point on a run where $\|\alpha\|$ holds satisfies $\alpha$ and any point where $\|\beta\|$ holds satisfies $\beta$, which will be used in the proofs without being mentioned explicitly every single time.
Proof: For $\mathbf{O p} \in\{\wedge, \vee\}$. Clearly, all runs are preserved. Also, by the constraint that $\|\alpha \mathbf{O p} \beta\| \Leftrightarrow\|\alpha\|$ Op $\|\beta\|$, it immediately follows that for any $i \in \mathbb{N}$ we have $\forall t \in I_{i}$ : $\left.s_{i}(\| \alpha \mathbf{O p} \beta]\right) \Rightarrow\left(\sigma^{(i, t)}=\alpha \mathbf{O p} \beta\right)$.

Proof: For $\mathbf{O p}=\mathbf{U}^{\mathrm{s}}$. Clearly, all the traces are preserved in $\mathcal{S}_{\alpha \mathbf{U}^{\mathrm{s}} \beta}$ as setting $\left\|\alpha \mathbf{U}^{\mathrm{s}} \beta\right\|$ to false leads to both constraints being satisfied regardless of the trace.

Now take a $\mathcal{S}_{\alpha \mathbf{U}^{\mathrm{s}} \beta}$ run $\tau=s_{0} s_{1} s_{2} \ldots$ with $\operatorname{trace}(\tau)=$ $\sigma=\left\langle I_{0} v_{0}\right\rangle\left\langle I_{1}, v_{1}\right\rangle \ldots, i \in \mathbb{N}$ and $t \in I_{i}$ with $s_{i}\left(\left\|\alpha \mathbf{U}^{\mathrm{s}} \beta\right\|\right)=$ true. It remains to show that $\sigma^{(i, t)} \models \alpha \mathbf{U}^{\mathrm{s}}$ $\beta$.

If $I_{i}$ is open, then by Constraint 1 we know that $s_{i}(\|\alpha\|)=$ true. Furthermore, there are three possibilities (multiple of which may be applicable):

1) $s_{i}(\|\beta\|)=$ true. In this case we can pick any future time point on the open interval $i$ and demonstrate that $\beta$ holds there and $\alpha$ holds up to that point, meaning that $\sigma^{(i, t)} \models \alpha \mathbf{U}^{\mathrm{s}} \beta$.

2) $s_{i+1}(\|\beta\|)=$ true. As $I_{i}$ is open, we know that $I_{i+1}$ is a singleton. Furthermore, as $s_{i}(\|\alpha\|)=$ true we know that $\alpha$ holds up to the single time point constituting $I_{i+1}$. Hence, $\sigma^{(i, t)}=\alpha \mathbf{U}^{\mathrm{s}} \beta$.

3) $s_{i}(\|\alpha\|)=$ true and $s_{i}(\|\beta\|)=s_{i+1}(\|\beta\|)=$ false. By Constraint 1 , then $\left.s_{i+1}\left(\| \alpha \mathbf{U}^{\mathrm{s}} \beta\right] \|\right)=$ true. By the fairness constraint $\mathcal{F}_{\alpha \mathbf{U}^{\mathrm{s}} \beta}$ we know that there is a future interval on which either $\|\beta\|$ holds or $\left\|\alpha \mathbf{U}^{\mathrm{s}} \beta\right\|$ does not hold. Pick $j>i+1$ as small as possible, such that $s_{j}(\|\beta\|)=$ true or $s_{j}\left(\left\|\alpha \mathbf{U}^{\mathrm{s}} \beta\right\|\right)=$ false. Now $s_{i}\left(\left\|\alpha \mathbf{U}^{\mathrm{s}} \beta\right\|\right)=\ldots=s_{j-1}\left(\left\|\alpha \mathbf{U}^{\mathrm{s}} \beta\right\|\right)=$ true and $s_{i}(\|\beta\|)=\ldots=s_{j-1}(\|\beta\|)=$ false. Note that the only way to satisfy Constraints 1 and 2 on intervals $i, \ldots, j-2$ now is by $\|\alpha\|^{\prime}$ holding on those intervals, meaning that $s_{i+1}(\|\alpha\|)=\ldots=s_{j-1}(\|\alpha\|)=$ true. Now

- If $I_{j-1}$ is open, then by Constraint 1 we know that $s_{j}(\|\beta\|)=$ true or $s_{j}\left(\left\|\alpha \mathbf{U}^{\mathrm{s}} \beta\right\|\right)=$ true. As we picked $j$ so that $s_{j}(\|\beta\|)=$ true or $s_{j}\left(\left\|\alpha \mathbf{U}^{\mathrm{s}} \beta\right\|\right)=$ false, we know that $s_{j}(\|\beta\|)=$ true (meaning that $\beta$ holds at interval $j$ ) in either case. Furthermore, as $I_{j-1}$ is open we know that $I_{j}$ is a singleton, implying that $\|\alpha\|$ (and thus $\alpha$ ) holds anywhere in between $(i, t)$ and $I_{j}$. Thus, $\sigma^{(i, t)} \models \alpha \mathbf{U}^{\mathrm{s}} \beta$.

- If $I_{j-1}$ is a singleton, then by Constraint 2 we know that either $s_{j}(\|\beta\|)=\operatorname{true}$ and $I_{j}$ is a singleton or $s_{j}\left(\left\|\alpha \mathbf{U}^{\mathrm{s}} \beta\right\|\right)=s_{j}(\|\alpha\|)=$ true. Again, by the choice of $j$ we know that $s_{j}\left(\| \alpha \mathbf{U}^{\mathrm{s}} \beta \rrbracket\right)=$ true implies that $s_{j}(\|\beta\|)=$ true. Thus, there is in either case a time point in interval $j$ at which $\|\beta\|$ (and thus $\beta$ ) holds such that $\|\alpha\|$ (and thus $\alpha$ ) holds anywhere in between $(i, t)$ and that time point. Hence, $\sigma^{(i, t)} \models \alpha \mathbf{U}^{\mathrm{s}} \beta$. 
If, in contrast, $I_{i}$ is a singleton, then by Constraint 2 there are two possibilities:

1) $I_{i+1}$ is a singleton and $s_{i+1}(\|\beta\|)=$ true. In this case, trivially $\sigma^{(i, t)}=\alpha \mathbf{U}^{\mathrm{s}} \beta$.

2) $s_{i+1}(\|\alpha\|)=s_{i+1}\left(\left\|\alpha \mathbf{U}^{\mathrm{s}} \beta\right\|\right)=$ true. If additionally $s_{i+1}(\|\beta\|)=$ true, then $\sigma^{(i, t)}=\alpha \mathbf{U}^{\mathrm{s}} \beta$ indiscriminately of whether $I_{i+1}$ is a singleton or an open interval. If, in contrast, $s_{i+1}(\|\beta\|)=$ false, then we can, again, pick $j>i+1$ as small as possible, such that $s_{j}(\|\beta\|)=$ true or $s_{j}\left(\left\|\alpha \mathbf{U}^{\mathrm{s}} \beta\right\|\right)=$ false. By proceeding precisely in the same way as in the Case 3 for open $I_{i}$, we can again deduce that $\sigma^{(i, t)} \models \alpha \mathbf{U}^{\mathrm{s}} \beta$.

Thus, $\sigma^{(i, t)} \models \alpha \mathbf{U}^{\mathrm{s}} \beta$ holds in each of the described cases.

Proof: For $\mathbf{O p}=\mathbf{F}_{\leq 0}^{\mathrm{s}}$.

Again, the "preservation of traces" property follows from the fact that the constraint is trivially satisfied globally when $\left|\left[\mathbf{F}_{\leq 0}^{\mathrm{s}} \alpha\right]\right|$ is set to false globally.

Now take a $\mathcal{S}_{\mathbf{F}_{\leq 0}^{\mathrm{s}}} \alpha$ run $\tau=s_{0} s_{1} s_{2} \ldots$ with $\operatorname{trace}(\tau)=$ $\sigma=\left\langle I_{0} v_{0}\right\rangle\left\langle I_{1}, v_{1}\right\rangle \ldots, i \in \mathbb{N}$ and $t \in I_{i}$ with $s_{i}\left(\left|\left[\mathbf{F}_{\leq 0}^{\mathrm{s}} \alpha\right]\right|\right)=$ true. It remains to show that $\sigma^{(i, t)} \models$ $\mathbf{F}_{\leq 0}^{\mathrm{s}} \alpha$.

By Constraint 3, we now know that $I_{i}$ and $I_{i+1}$ are both singletons. This, in particular, means that $I_{i+1}=[t, t]$. Furthermore, by Constraint 3 one of the following holds:

- $s_{i+1}(\|\alpha\|)=$ true. In this case, $\sigma^{(i, t)} \models \mathbf{F}_{\leq 0}^{\mathrm{s}} \alpha$ trivially.

- $s_{i+1}\left(\left|\left[\mathbf{F}_{\leq 0}^{\mathrm{s}} \alpha\right]\right|\right)=$ true. In this case, $I_{i+2}$ is a singleton as well and again $\left.s_{i+2}(\| \alpha]\right)=$ true or $s_{i+2}\left(\left|\left[\mathbf{F}_{<0}^{\mathrm{s}} \alpha\right]\right|\right)=$ true. Applying this argument repeatedly leads to the conclusion, that there needs to be an interval on which $\|\alpha\|$ holds before the next open interval. The fact that $\sigma$ is non-zeno, furthermore, implies that there is a future open interval. Thus, we can conclude that there is a sequence of singleton intervals starting at interval $i$ such that $\| \alpha \rrbracket$ (and thus $\alpha$ ) holds on the last interval in that sequence. Thus, $\sigma^{(i, t)}=\mathbf{F}_{\leq 0}^{\mathrm{s}} \alpha$.

In both cases we were able to demonstrate that $\sigma^{(i, t)}=$ $\mathbf{F}_{\leq 0}^{\mathrm{s}} \alpha$.

Proof: For $\mathbf{O p}=\mathbf{F}_{<n}^{\mathrm{s}}$. The "preservation of traces" property follows from the fact that the constraints can easily be satisfied globally when $\left\|\mathbf{F}_{<n}^{\mathrm{s}} \alpha\right\|$ is set to false globally.

Now take a $\mathcal{S}_{\mathbf{F}_{<n}^{\mathrm{s}} \alpha}$ run $\tau=s_{0} s_{1} s_{2} \ldots$ with $\operatorname{trace}(\tau)=$ $\sigma=\left\langle I_{0} v_{0}\right\rangle\left\langle I_{1}, v_{1}\right\rangle \ldots, i \in \mathbb{N}$ and $t \in I_{i}$ with $s_{i}\left(\left\|\mathbf{F}_{<n}^{\mathrm{s}} \alpha\right\|\right)=$ true. It remains to show that $\sigma^{(i, t)} \models \mathbf{F}_{<n}^{\mathrm{s}} \alpha$.

Choose $j \in \mathbb{N}$ as large as possible such that $0 \leq j \leq i$ and either $R_{c}$ holds at interval $j-1$ or $j=0$. We now know that (i) $s_{j}(c)=0$ and (ii) $s_{j}$ (lefto) $=$ true iff $I_{j}$ is open. Let $\bar{j}=j+1$ if interval $j$ is a singleton and $\bar{j}=j$ otherwise. If now $j<i$, then we know that $R_{c}$ does not hold on interval $i-1$ meaning that $s_{i-1}\left(\left\|\mathbf{F}_{<n}^{\mathrm{s}} \alpha\right\|\right)=$ true, $s_{i}(\|\alpha\|)=$ false and $s_{i-1}(\|\alpha\|)=$ false if $I_{i-1}$ is open. Applying the same reasoning repeatedly, we can deduce that, firstly, $\left.s_{j}\left(\| \mathbf{F}_{<n}^{\mathrm{s}} \alpha\right] \|\right)=\ldots=s_{i}\left(\| \mathbf{F}_{<n}^{\mathrm{s}} \alpha \rrbracket\right)=$ true and, secondly, $s_{\bar{j}}(\|\alpha\|)=\ldots=s_{i}(\|\alpha\|)=$ false.

Let $\bar{i}=i+1$ if $I_{i}$ is a singleton and $\bar{i}=i$ otherwise. Now assume that $s_{\bar{i}}(\| \alpha \rrbracket)=s_{\bar{i}+1}(\|\alpha\|)=\ldots=$ false. In this case Constraints 4 and 5 imply that $\left.s_{i}\left(\| \mathbf{F}_{<n}^{\mathrm{s}} \alpha\right] \|\right)=$ $s_{\bar{i}+1}\left(\left\|\mathbf{F}_{<n}^{\mathrm{s}} \alpha\right\|\right)=\ldots=$ true. Thus $R_{c}$ is false on intervals $\bar{i}, \bar{i}+1, \ldots$. As we assumed a non-zeno trace, this implies that there is no upper bound to the value of $c$ on the intervals $\bar{i}, \bar{i}+1, \ldots$ implying that $T_{c}$ eventually becomes false on all intervals starting from some interval after interval $\bar{i}$. As now $\left\|\mathbf{F}_{<n}^{\mathrm{s}} \alpha\right\|$ holds globally and $\|\alpha\|$ globally does not hold starting from interval $\bar{i}$, this contradicts Constraint 9. Thus, assuming there is no point at which $\|\alpha\|$ holds after interval $\bar{i}$ leads to a contradiction, implying that there has to be a point where $\|\alpha\|$ holds. Thus, we can pick $k \geq \bar{i}$ as small as possible such that $\left.s_{k}(\| \alpha]\right)=$ true.

- Case 1: $i=k$. As $k \geq \bar{i}$, this implies that $I_{i}$ is open. As, furthermore, $s_{k}(\|\alpha\|)=$ true and thus $s_{i}(\|\alpha\|)=$ true we know that $\sigma^{(i, t)} \models \mathbf{F}_{<n}^{\mathrm{s}}\|\alpha\|$.

- Case 2: $i \neq k$ and $k=j+1$. As $j \leq i \leq k$ we know know that $i=j$. Now

- If $I_{i}$ is a singleton, then $\sigma^{(i, t)} \models \mathbf{F}_{<n}^{\mathrm{s}}\|\alpha\|$ trivially holds.

- If $I_{i}$ is open, then $\bar{i}=i$. Thus, by the choice of $k$ we know that $s_{i}(\|\alpha\|)=$ false. As $s_{i}\left(\left\|\mathbf{F}_{<n}^{\mathrm{s}} \alpha\right\|\right)=$ true, Constraint 9 implies that $T_{c}$ holds at interval $i$. As $j=i$, we know that $s_{i}(c)=0$. By the definition of $T_{c}$, we now know that the value of $\delta$ at interval $i$ is less than or equal to $n$. This together with the fact that $I_{i}$ is open implies we can pick a point in $I_{k}$ that is less than $n$ time units away from $(i, t)$ and, ultimately, that $\sigma^{(i, t)} \models \mathbf{F}_{<n}^{\mathrm{s}} \| \alpha \rrbracket$.

- Case 3: $i \neq k$ and $k \geq j+2$. By $s_{i}(\| \alpha \rrbracket)=\ldots=$ $s_{k-1}(\|\alpha\|)=$ false and by Constraints 4 and 5, we now know that $s_{i+1}\left(\left\|\mathbf{F}_{<n}^{\mathrm{s}} \alpha\right\|\right)=\ldots=s_{k-1}\left(\left\|\mathbf{F}_{<n}^{\mathrm{s}} \alpha\right\|\right)=$ true. Together with our previous observations we now know that $s_{j}(\|\alpha\|)=\ldots=s_{k-1}(\|\alpha\|)=$ false and $s_{j}\left(\left\|\mathbf{F}_{<n}^{\mathrm{s}} \alpha\right\|\right)=\ldots=s_{k-1}\left(\left\|\mathbf{F}_{<n}^{\mathrm{s}} \alpha\right\|\right)=$ true. This implies that $R_{c}$ does not hold on intervals $j, \ldots, k-2$, in turn implying that $c$ and lefto are updated according to Constraint 8 on the transitions from intervals $j, \ldots, k-2$ to the respective following interval. Therefore, $s_{k-1}(c)$ is the difference between the left bound of $I_{j}$ and the left bound of $I_{k-1}$. Thus, $s_{k-1}(c)+s_{k-1}(\delta)$ is the difference between the left bound of $I_{k}$ and the left bound of $I_{j}$. As $s_{k-1}\left(\left\|\mathbf{F}_{<n}^{\mathrm{s}} \alpha\right\|\right)=$ true and $s_{k-1}(\| \alpha \rrbracket)=$ false, Constraint 9 implies that $T_{c}$ holds at interval $k-1$. Now

- If $I_{j}$ is open, then the difference between the left bounds of $I_{k}$ and $I_{j}$ is less than or equal to $n$. Then we can for every point in $I_{j}$ pick another point in $I_{k}$ that is less than $n$ time units away from the point in $I_{j}$. 
- If $I_{j}$ is a singleton, then the difference between the left bounds of $I_{k}$ and $I_{j}$ is less than $n$. Again, this means that we can for every point in $I_{j}$ pick another point in $I_{k}$ that is less than $n$ time units away from the point in $I_{j}$.

Finally, as $j \leq i \leq k$, we can also for $(i, t)$ pick a point in $I_{k}$ that is less than $n$ time units away. As $s_{k}(\|\alpha\|)=$ true, this implies that $\sigma^{(i, t)} \models \mathbf{F}_{<n}^{\mathrm{s}} \alpha$.

In each case we were able to demonstrate that $\sigma^{(i, t)} \models$ $\mathbf{F}_{<n}^{\mathrm{s}} \alpha$.

Proof: For $\mathbf{O p}=\mathbf{F}_{<n}^{\mathrm{s}}$. The proof for $\mathbf{O p}=\mathbf{F}_{<n}^{\mathrm{s}}$ proceeds precisely as the proof for $\mathbf{O p}=\mathbf{F}_{<n}^{\mathrm{s}}$, except for arguing that there are time points $\leq n$ time units apart in $I_{j}$ and $I_{k}$ in Case 3 .

By the definition of $T_{c}$ for $\triangleleft=<$ and as $T_{c}$ holds at interval $k-1$ we know that in Case 3 one of the following:

- The difference between the left bound of $I_{j}$ is less than $n$ time units. In this case, we can trivially pick for any point in in $I_{j}$ a point that is $\leq n$ time units away in $I_{k}$.

- The difference between the left bound of $I_{j}$ is $n$ time units and $I_{j}$ open or $I_{k}$ is a singleton. Again we can pick for any point in in $I_{j}$ a point that is $\leq n$ time units away in $I_{k}$.

Proof: For $\mathbf{O p}=\mathbf{U}_{>n}^{\mathrm{s}}$. The "preservation of traces" property follows from the fact that the constraints can easily be satisfied globally when $\| \alpha \mathbf{U}_{>n}^{\mathrm{s}} \beta \rrbracket$ and oblig are set to false globally.

Now take a $\mathcal{S}_{\alpha \mathbf{U}_{>n}^{\mathrm{s}} \beta}$ run $\tau=s_{0} s_{1} s_{2} \ldots$ with $\operatorname{trace}(\tau)=$ $\sigma=\left\langle I_{0} v_{0}\right\rangle\left\langle I_{1}, v_{1}\right\rangle \ldots, i \in \mathbb{N}$ and $t \in I_{i}$ with $s_{i}\left(\left\|\alpha \mathbf{U}_{>n}^{\mathrm{s}} \beta\right\|\right)=$ true. It remains to show that $\sigma^{(i, t)} \models$ $\alpha \mathbf{U}_{>n}^{\mathrm{s}} \beta$.

By the fact that $s_{i}\left(\left\|\alpha \mathbf{U}_{>n}^{\mathrm{s}} \beta\right\|\right)=$ true and Constraint 10 we know that $s_{i}$ (oblig) $=$ true. Let $\bar{i}=i$ if $I_{i}$ is open and $\bar{i}=i+1$ otherwise. Now

- Case 1: $\hat{r}$ holds on interval $i+1$. Then $T_{c}$ and $\|\beta\|$ hold on interval $i+1$. As $s_{i}\left(\left\|\alpha \mathbf{U}_{>n}^{\mathrm{s}} \beta\right\|\right)=$ true, we know that $s_{i+1}(c)=0$ and $s_{i+1}($ righto $)=$ true iff $I_{i}$ is open. Now

- If interval $I_{i+1}$ is a singleton, then $s_{i+1}(c)+$ $s_{i+1}(\delta)=0$. Because $T_{c}$ holds on interval $i+1$, we then know that $n=0$ and $s_{i+1}$ (righto) $=$ true, the latter implying that $I_{i}$ is open. Now Constraint 12 implies that $s_{i}(\|\alpha\|)=$ true. Furthermore, as $I_{i}$ is open, we can pick a point that is more than 0 time units away from $(i, t)$ in $I_{i+1}$. Thus, $\sigma^{(i, t)} \models \alpha \mathbf{U}_{>n}^{\mathrm{s}} \beta$.

- If, in contrast, $I_{i+1}$ is open then $I_{i}$ is a singleton and $s_{i+1}($ righto $)=$ false. Thus, the fact that $T_{c}$ is satisfied on interval $i+1$ implies that $s_{i+1}(\delta)>n$. Thus we can pick a point that is more than $n$ time units away from $(i, t)$ in $I_{i+1}$. As $I_{i}$ is a singleton and $I_{i+1}$ an open interval, Constraint 13 implies that $s_{i+1}(\|\alpha\|)=$ true, meaning that $\sigma^{(i, t)} \models \alpha \mathbf{U}_{>n}^{\mathrm{s}} \beta$.

- Case 2: $\hat{r}$ does not hold on interval $i+1$ and $s_{i+1}($ oblig $)=$ false. Now based on Constraint 11 this means that $n=0$. Furthermore, the only way to satisfy Constraints 12 and 13 is if $I_{i}$ is open and $\hat{r}$ and $\|\alpha\|$ hold at interval $i$. In this case, $s_{i}(\|\alpha\|)=s_{i}(\|\beta\|)=$ true, implying that $\sigma^{(i, t)}=\alpha \mathbf{U}_{>0}^{\mathrm{s}} \beta$.

- Case 3: $\hat{r}$ does not hold on interval $i+1$ and $s_{i+1}(\|\alpha\|)=$ false. Again, Constraint 11 implies that $n=0$. Furthermore, Constraints 12 and 13 can only be satisfied if $I_{i}$ is open and both $\alpha$ and $\hat{r}$ hold on interval $i$. Clearly, $\sigma^{(i, t)} \models \alpha \mathbf{U}_{>0}^{\mathrm{s}} \beta$.

- Case 4: $\hat{r}$ does not hold on interval $i+1, s_{i+1}($ oblig $)=$ $s_{i+1}(\|\alpha\|)=$ true and $\hat{r}$ holds on interval $i+2$ or any later interval. Pick $j$ as small as possible such that $j \geq i+2$ and $\hat{r}$ holds at interval $j$. Let $\bar{j}=j$ if $I_{j}$ is open and $\bar{j}=j-1$ if $I_{j}$ is a singleton. Note that Constraints 12 and 13 correspond to Constraints 1 and 2 in the $\mathbf{U}^{\mathrm{s}}$-encoding, except that $\left\|\alpha \mathbf{U}_{>n}^{\mathrm{s}} \beta\right\|$ has been replaced by oblig and $\|\beta\|$ has been replaced by $\hat{r}$. This correspondence allows us to conclude that $\alpha \mathbf{U}^{\mathrm{s}} \hat{r}$ is satisfied everywhere on interval $i+1$. By the fact that $\alpha \mathbf{U}^{\mathrm{s}} \hat{r}$ holds on interval $i+1$ and the choice of $j$ we now know that $s_{i+2}(\|\alpha\|)=\ldots=s_{\bar{j}}(\|\alpha\|)=$ true. Furthermore, we assumed $s_{i+1}(\|\alpha\|)=$ true. Also, if $I_{i}$ is open, the fact that $s_{i}$ (oblig) $=$ true and Constraint 12 imply that $s_{i}(\|\alpha\|)=$ true. Thus, we know that $s_{\bar{i}}(\|\alpha\|)=\ldots=s_{\bar{j}}(\|\alpha\|)=$ true.

Now choose $k$ as large as possible such that $i \leq k<j$ and $s_{k}\left(\left\|\alpha \mathbf{U}_{>n}^{\mathrm{s}} \beta\right\|\right)=$ true. Now the values of $c$ and righto are set according to Constraint 14 on interval $k+1$ and and according to Constraint 15 on intervals $k+2, \ldots j$. This implies that $s_{j}(c)$ is the difference between the left bound of $I_{j}$ and the right bound of $I_{k}$. Thus, $s_{j}(c)+s_{j}(\delta)$ is the difference between the right bounds of $I_{j}$ and $I_{k}$. Furthermore, $s_{j}($ righto $)=$ true iff $I_{k}$ is open. As $\hat{r}$ holds on interval $j$, we know by the definition of $\hat{r}$ that $T_{c}$ holds on interval $j$. Thus, the difference between the right bounds of $I_{j}$ and $I_{k}$ is greater or equal to $n$ and greater than $n$ if $I_{k}$ is a singleton. This implies, that for every point in $I_{k}$ we can pick a point in $I_{j}$ that is more than $n$ time units away. As $i \leq k$, we can also pick a point in $I_{j}$ that is more than $n$ time units away from $t$.

Furthermore, by $\hat{r}$ holding on interval $j$ we know that $s_{j}(\|\beta\|)=$ true. Together with the fact that $s_{\bar{i}}(\|\alpha\|)=\ldots=s_{\bar{j}}(\|\alpha\|)=$ true, this implies that $\sigma^{(i, t)} \models \alpha \mathbf{U}_{>n}^{\mathrm{s}} \beta$.

- Case 5: $\hat{r}$ does not hold on intervals $i+1, i+2, \ldots$ and $s_{i+1}($ oblig $)=s_{i+1}(\|\alpha\|)=$ true. Now by Constraints 12 and 13 we know that $s_{\bar{i}}(\|\alpha\|)=s_{\bar{i}+1}(\|\alpha\|)=$ 
$\ldots=$ true and $s_{\bar{i}}($ oblig $)=s_{\bar{i}+1}($ oblig $)=\ldots=$ true, i.e. $\|\alpha\|$ and oblig hold globally starting from interval $\bar{i}$. Thus, $\neg$ oblig holds only on finitely many intervals. By fairness constraint $\mathcal{F}_{\alpha \mathbf{U}_{>n}^{\mathrm{s}} \beta}$, this implies that $\|\beta\|$ holds on infinitely many intervals. As $\sigma$ is non-zeno, this implies that we can pick an interval that contains a point more than $n$ time units away from $(i, t)$ and on which $\|\beta\|$ holds. As $\|\alpha\|$ holds globally starting from interval $\bar{i}$, this implies that $\sigma^{(i, t)} \models \alpha \mathbf{U}_{>n}^{\mathrm{s}} \beta$.

In each case, we were able to demonstrate that $\sigma^{(i, t)} \models$ $\alpha \mathbf{U}_{>n}^{\mathrm{s}} \beta$.

Proof: For $\mathbf{O p}=\mathbf{U}_{\geq n}^{\mathrm{s}}$. As a first observation, we note that in case of $\mathbf{O p}=\mathbf{U}_{\geq n}^{\mathrm{s}}$ we know that $n>0$, as we use the $\mathbf{U}^{\mathrm{s}}$ encoding to encode $\alpha \mathbf{U}_{>0}^{\mathrm{s}} \beta$.

We obtain the $\mathbf{O p}=\mathbf{U}_{\geq n}^{\mathrm{s}}$ proof from the $\mathbf{O p}=\mathbf{U}_{>n}^{\mathrm{s}}$ proof by the following modifications:

- In Case 1, assuming $I_{i+1}$ to be a singleton contradicts our observation that $n>0$, meaning that $I_{i+1}$ is open, $I_{i}$ is a singleton. Now the fact that $T_{c}$ holds on interval $i+1$ implies that $s_{i+1}(\delta)>n$. This allows us to to pick a point in $I_{i}$ that is $\geq n$ time units away from $(i, t)$. Analogously to the $\mathbf{O p}=\mathbf{U}_{>n}^{\mathrm{s}}$ proof this leads to $\sigma^{(i, t)} \models \alpha \mathbf{U}_{\geq n}^{\mathrm{s}} \beta$.

- Cases 2 and 3 contradict $n>0$.

- In Case 4, the fact that $T_{c}$ holds on interval $j$ implies that either (i) the difference between the right bounds of $I_{j}$ and $I_{k}$ is greater than $n$ or (ii) the right bounds of $I_{j}$ and $I_{k}$ equals $n$ and $I_{k}$ is open or $I_{j}$ is a singleton. Thus, we can for every point in $I_{k}$ pick a point in $I_{j}$ that is $\geq n$ time units away.

- Case 5 does not need modification.

Proof: For $\mathbf{O p}=\mathbf{R}^{\mathrm{s}}$. The "preservation of traces" property follows from the fact that the constraints can easily be satisfied globally when $\left\|\alpha \mathbf{R}^{\mathrm{s}} \beta\right\|$ and oblig are set to false globally.

Now take a $\mathcal{S}_{\alpha \mathbf{R}^{\mathrm{s}} \beta}$ run $\tau=s_{0} s_{1} s_{2} \ldots$ with $\operatorname{trace}(\tau)=$ $\sigma=\left\langle I_{0} v_{0}\right\rangle\left\langle I_{1}, v_{1}\right\rangle \ldots, i \in \mathbb{N}$ and $t \in I_{i}$ with $s_{i}\left(\left\|\alpha \mathbf{R}^{\mathrm{s}} \beta\right\|\right)=$ true. It remains to show that $\sigma^{(i, t)} \models \alpha \mathbf{R}^{\mathrm{s}}$

$\beta$. Let $\bar{i}=i$ if $I_{i}$ is open and $\bar{i}=i+1$ if $I_{i}$ is a singleton.

As a first case assume $s_{\bar{i}}(\|\alpha\|)=s_{\bar{i}+1}(\|\alpha\|)=\ldots=$ false. Then by Constraints 16,17 and 18 we know that $s_{\bar{i}}($ oblig $)=s_{\bar{i}+1}($ oblig $)=\ldots=$ true. Now Constraint 19 implies that $s_{\bar{i}}(\|\beta\|)=s_{\bar{i}+1}(\|\beta\|)=\ldots=$ true. Clearly, in this case $\sigma^{(i, t)} \models \alpha \mathbf{R}^{\mathrm{s}} \beta$.

As a second case assume $\|\alpha\|$ holds at interval $\bar{i}$ or any later interval. Then let $j \geq \bar{i}$ be as small as possible such that $s_{j}(\| \alpha \rrbracket)=$ true. Then by Constraints 16, 17 and 18, we know that $s_{\bar{i}}($ oblig $)=\ldots=s_{j}($ oblig $)=$ true. Now

- If $I_{j}$ is open, then by Constraint 19 we have that $s_{\bar{i}}(\|\beta\|)=\ldots=s_{j-1}(\|\beta\|)=$ true. This, in turn, implies that for any time point after $(i, t)$ at which $\|\beta\|$ does not hold there is an earlier time point in interval $j$. Hence, $\sigma^{(i, t)} \models \alpha \mathbf{R}^{\mathrm{s}} \beta$.
- If $I_{j}$ is a singleton, then by Constraint 19 we have that $s_{\bar{i}}(\|\beta\|)=\ldots=s_{j}(\|\beta\|)=$ true. Thus, the single time point in interval $j$, at which $\|\alpha\|$ holds, lies in between time point $(i, t)$ and any potential future point at which $\| \beta \rrbracket$ does not hold. Again, $\sigma^{(i, t)} \models \alpha \mathbf{R}^{\mathrm{s}} \beta$.

Proof: For $\mathbf{O p}=\mathbf{G}_{\leq 0}^{\mathrm{s}}$. The "preservation of traces" property follows from the fact that the constraints can easily be satisfied globally when $\left|\left[\mathbf{G}_{\leq 0}^{\mathrm{s}} \alpha\right]\right|$ is set to false globally.

Now take a $\mathcal{S}_{\mathbf{G}_{\leq 0}^{\mathrm{s}} \alpha}$ run $\tau=s_{0} s_{1} s_{2} \ldots$ with $\operatorname{trace}(\tau)=$ $\sigma=\left\langle I_{0} v_{0}\right\rangle\left\langle I_{1}, v_{1}\right\rangle \ldots, i \in \mathbb{N}$ and $t \in I_{i}$ with $s_{i}\left(\left|\left[\mathbf{G}_{\leq 0}^{\mathrm{s}} \alpha\right]\right|\right)=$ true. It remains to show that $\sigma^{(i, t)} \models$ $\mathbf{G}_{\leq 0}^{\mathrm{s}} \alpha$.

Recall, that by the semantics of $\mathbf{G}_{\leq n}^{\mathrm{s}}$, a point on a trace satisfies $\mathbf{G}_{\leq 0}^{\mathrm{s}} \alpha$ iff all future points that are zero time units away from that point satisfy $\alpha$. Note that a future time point can be zero time units away only if both the current and the next interval are singletons.

By Constraint 20, there are two possibilities:

- $I_{i}$ or $I_{i+1}$ is open. In this case, there are no future time points that are zero time units away for any point on interval $i$. Hence, $\sigma^{(i, t)} \models \mathbf{G}_{\leq 0}^{\mathrm{s}} \alpha$ trivially.

- $I_{i}$ and $I_{i+1}$ are both singletons and $\left.s_{i+1}(\|\alpha\|)=s_{i+1}\left(\| \mathbf{G}_{\leq 0}^{\mathrm{s}} \alpha\right] \|\right)=$ true. By the fact that $\left.s_{i+1}\left(\| \mathbf{G}_{\leq 0}^{\mathrm{s}} \leq 0\right]\right)=$ true, we can again deduce that either $I_{i+2}$ is open or $\left.s_{i+2}(\|\alpha\|)=s_{i+2}\left(\| \mathbf{G}_{\leq 0}^{\mathrm{s}} \alpha\right] \mid\right)=$ true. Repeatedly applying this argument leads to the conclusion that $\| \alpha \rrbracket$ has to hold on all future singletons up to the next open interval, i.e., all future intervals containing points zero time units away from $(i, t)$. Thus, $\sigma^{(i, t)} \models \mathbf{G}_{\leq 0}^{\mathrm{s}} \alpha$.

In both cases, we were able to show that $\sigma^{(i, t)} \models \mathbf{G}_{\leq 0}^{\mathrm{s}} \alpha$.

Proof: For $\mathbf{O p}=\mathbf{G}_{<n}^{\mathrm{s}}$. The "preservation of traces" property follows from the fact that the constraints can easily be satisfied globally when $\left\|\mathbf{G}_{<n}^{\mathrm{s}} \alpha\right\|$ and oblig are set to false globally.

Now take a $\mathcal{S}_{\mathbf{G}_{<n}^{\mathrm{s}} \alpha}$ run $\tau=s_{0} s_{1} s_{2} \ldots$ with $\operatorname{trace}(\tau)=$ $\sigma=\left\langle I_{0} v_{0}\right\rangle\left\langle I_{1}, v_{1}\right\rangle \ldots, i \in \mathbb{N}$ and $t \in I_{i}$ with $s_{i}\left(\left\|\mathbf{G}_{<n}^{\mathrm{s}} \alpha\right\|\right)=$ true. It remains to show that $\sigma^{(i, t)} \models \mathbf{G}_{<n}^{\mathrm{s}} \alpha$.

Take an arbitrary $j>i$ and $t_{2} \in I_{j}$ such that $t_{2}-t<n$ (if such an $j$ exists). Let $k$ be as large as possible such that $i \leq k<j$ and $s_{k}\left(\left\|\mathbf{G}_{<n}^{\mathrm{s}} \alpha\right\|\right)=$ true. By Constraint 22 , we know that $s_{k+1}(c)=0$. Furthermore, by Constraints 22 and 23, we know that $s_{j}(c)$ is the difference between the right bound of $I_{k}$ and the left bound $I_{j}$. As $k \geq i$ and $t_{2}-t<n$, we know that this difference must be less than $n$ and, consequently, $T_{c}$ holds on interval $j$. By Constraint 21 , this implies that $s_{j}(\| \alpha \rrbracket)=$ true. As we picked $j$ to be an arbitrary interval containing a point less than $n$ time units away from $(i, t)$, we can conclude that $\| \alpha \rrbracket$ holds at any point on intervals $i+1, i+2, \ldots$ that is less than $n$ time units after $(i, t)$ 
Additionally, Constraint 21 ensures that $\|\alpha\|$ also holds on interval $i$, if that interval is open. Thus, it is guaranteed that $\|\alpha\|$ holds at all future points less than $n$ time units away and $\sigma^{(i, t)}=\mathbf{G}_{<n}^{\mathrm{s}} \alpha$.

Proof: For $\mathbf{O p}=\mathbf{G}_{\leq n}^{\mathrm{s}}$. We modify the proof for $\mathbf{G}_{<n}^{\mathrm{s}}$ by picking $j>i, t_{2} \in I_{j}$ with $t_{2}-t \leq n$. Then we know that the difference between the left bound of $I_{j}$ and the right bound of $I_{i}$ is less than or equal to $n$ and can be equal to $n$ only if both intervals are singletons. Now

- if $s_{j}(c)<n$ then $T_{c}$ is satisfied on interval $j$ and we proceed as before.

- if $s_{j}(c)=n$ then we know that $s_{j}(c)$ is precisely the difference between the left bound of $I_{j}$ and the right bound of $I_{i}$ and $I_{i}$ and $I_{j}$ are both singletons. Now, $k=i$ or there is a sequence of intervals $I_{i} I_{i+1} \ldots I_{k}$ that are all singletons. In either case, $I_{k}$ is a singleton and $s_{j}$ (righto) $=$ false. Thus, $T_{c}$ is satisfied also in this case and we continue as before.

Proof: For $\mathbf{O p}=\mathbf{R}_{>n}^{\mathrm{s}}$. The "preservation of traces" property follows from the fact that the constraints can easily be satisfied globally when $\left\|\alpha \mathbf{R}_{>n}^{\mathrm{s}} \beta\right\|$ and oblig are set to false globally.

Now take a $\mathcal{S}_{\alpha \mathbf{R}_{>n}^{\mathrm{s}} \beta} \beta$ run $\tau=s_{0} s_{1} s_{2} \ldots$ with $\operatorname{trace}(\tau)=$ $\sigma=\left\langle I_{0} v_{0}\right\rangle\left\langle I_{1}, v_{1}\right\rangle \ldots, i \in \mathbb{N}$ and $t \in I_{i}$ with $s_{i}\left(\left\|\alpha \mathbf{R}_{>n}^{\mathrm{s}} \beta\right\|\right)=$ true. It remains to show that $\sigma^{(i, t)} \models$ $\alpha \mathbf{R}_{>n}^{\mathrm{s}} \beta$. As usual, let $\bar{i}=i$ if interval $i$ is open and $\bar{i}=i+1$ otherwise.

As a first case assume that for all $\left(j, t_{2}\right) \in T(\sigma)$ with $t_{2}-t>n$ we have that $s_{j}(\|\beta\|)=$ true. In this case, we trivially have $\sigma^{(i, t)}=\alpha \mathbf{R}_{>n}^{\mathrm{s}} \beta$.

As a second case, assume there is $\left(j, t_{2}\right) \in T(\sigma)$ with $t_{2}-t>n$ and $s_{j}(\|\beta\|)=$ false.

- Case 2.a: $i=j$ : As $t_{2}-t>n$, this implies that $\delta>n$ at interval $i$. Then, Constraint 28 implies that $s_{i}(\|\alpha\|)=$ true. As interval $i$ also has to be open to allow $\delta$ to be non-zero, we now know that there is point in between $(i, t)$ and $\left(j, t_{2}\right)$ where $\| \alpha \rrbracket$ holds.

- Case 2.b: $i<j$ and $s_{j}$ (oblig) = false. Analogously to the proof of the lemma for the untimed $\mathbf{R}^{\mathrm{s}}$ encoding, $s_{j}($ oblig $)=$ false implies that $\|\alpha\|$ holds at some point in between $(i, t)$ and $\left(j, t_{2}\right)$.

- Case 2.c: $i<j$ and $s_{j}($ oblig $)=s_{j}(\|\alpha\|)=$ true and $I_{j}$ is open. Then, as $I_{j}$ is open and $\left.s_{j}(\| \alpha]\right)=$ true, we know that $\|\alpha\|$ holds at some point in between $(i, t)$ and $\left(j, t_{2}\right)$.

- Case 2.d: $i<j$ and $s_{j}($ oblig $)=$ true and either $I_{j}$ is a singleton or $s_{j}(\| \alpha \rrbracket)=$ false. Then, according to Constraints 27 we know $T_{c}$ does not hold on interval $j$. Now pick $k \leq j$ as large as possible such that $s_{k}(c)=0$ and one of the following:

- $D_{c}$ holds at interval $k-1$

- $R_{c}$ holds at interval $k-1$, or

$$
-k=0
$$

Now Constraint 31 implies that $s_{j}(c)$ is the difference between the left bound of $I_{j}$ and the left bound of $I_{k}$. Consequently, $s_{j}(c)+s_{j}(\delta)$ is the difference between the right bound of $I_{j}$ and the left bound of $I_{k}$. Now by the fact that $T_{c}$ does not hold we know that the difference between the right bound of $I_{j}$ and the left bound of $I_{k}$ is less than or equal to $n$ time units. Furthermore, $t_{2}-t>n$ implies that the difference between the right bound of $I_{j}$ and the left bound of $I_{i}$ is greater than $n$. This, in turn, implies that $k>i$ and at least one of the intervals $I_{i}, I_{i+1}, \ldots, I_{k-1}$ is open. This implies that $k>\bar{i}>0$ Now, there are three possibilities:

- $R_{c}$ holds at interval $k-1$ and $s_{k-1}(\|\alpha\|)=$ true Then there is a point in between $(i, t)$ and $\left(j, t_{2}\right)$ at which $\alpha$ holds.

- $R_{c}$ holds at interval $k-1$ and $s_{k-1}(\|\alpha\|)=$ false. By the definition of $R_{c}$ now $s_{k-1}($ oblig $)=$ false. analogously to the untimed $\mathbf{R}^{\mathrm{s}}$ encoding and Case 2.b, this implies that $\|\alpha\|$ holds on an interval in the range $\bar{i}, \ldots, k-1$.

- $D_{c}$ holds at interval $k-1$. This implies that $s_{k-1}(\|\alpha\|)=$ true

In each of the mentioned cases, we were able to show $\|\alpha\|$ holds at some point in between $(i, t)$ and $\left(j, t_{2}\right)$, allowing us to conclude that $\sigma^{(i, t)} \models \alpha \mathbf{R}_{>n}^{\mathrm{s}} \beta$.

Proof: For Op $=\mathbf{R}_{\geq n}^{\mathrm{s}}$. To adapt the $\mathbf{R}_{>n}^{\mathrm{s}}$ proof for $\mathbf{R}_{\geq n}^{\mathrm{s}}$ modify the second case as follows. We pick a $\left(j, t_{2}\right) \in T(\sigma)$ with $s_{j}(\|\beta\|)=$ false and $t_{2}-t \geq n$. Cases 2.a to 2.c do not require substantial changes. In Case 2.d, we pick $k$ as before.

We now observe that $t_{2}-t \geq n$ implies that the difference between the right bound of $I_{j}$ and the left bound of $I_{i}$ is greater than or equal to $n$. Furthermore, the difference can only be equal if $I_{i}$ and $I_{j}$ are both singletons.

The fact that $T_{c}$ does not hold at interval $j$ implies that $s_{j}(c)+s_{j}(\delta) \leq n$. Furthermore, if $s_{j}(c)+s_{j}(\delta)=n$ then we know that $I_{j}$ is open or $s_{j}$ (lefto) $=$ true.

Now

- If the difference between the right bound of $I_{j}$ and the left bound of $I_{i}$ is greater than $n$, we again can conclude that $k>\bar{i}$ and proceed as before.

- Likewise, if $s_{j}(c)+s_{j}(\delta)<n$ we conclude that $k>\bar{i}$ and proceed as before.

- If the difference between the right bound of $I_{j}$ and the left bound of $I_{i}$ equals $n$ and $s_{j}(c)+s_{j}(\delta)=n$, then our previous observations tell us that (i) $I_{i}$ and $I_{j}$ are singletons (ii) $s_{j}($ lefto $)=$ true. Furthermore, as $n>0, s_{j}(c)+s_{j}(\delta)=n, s_{k}(c)=0$ and $I_{j}$ is a singleton we know that $k<j$. As the value of lefto on intervals $k+1, \ldots, j$ set according to Constraint 31, we know that $s_{k}$ (lefto $)=$ true. This eliminates the 
possibilities that $k=0$ or that $D_{c}$ holds on interval $k-1$, leaving only the possibility that $R_{c}$ holds on interval $k-1$. Then by Constraint $30, I_{k}$ is open. Together with our assumptions about the left-boundto-right-bound differences and the fact that $I_{i}$ is a singleton, this implies that $k>i$. Finally, as $I_{i}$ is a singleton and $\left.s_{i}\left(\| \alpha \mathbf{R}_{\geq n}^{\mathrm{s}} \beta\right] \mid\right)=$ true, $R_{c}$ does not hold on interval $i$, meaning that $k>i+1=\bar{i}$. Now we can continue as previously.

\section{F. Completeness proofs}

Lemma 5: The transition system $\mathcal{S}_{p}$ is a complete encoding for $p, \mathcal{S}_{\neg p}$ is a complete encoding for $\neg p$. If a transition system $\mathcal{S}$ over $A P$ is a complete encoding of $\alpha$ and $\beta$, then the transition system $\mathcal{S}_{\mathbf{O p} \alpha}$ over $A P$ is a complete encoding of $\mathbf{O p} \alpha$ for each $\mathbf{O p} \in\left\{\mathbf{F}_{\leq 0}^{\mathrm{s}}, \mathbf{F}_{<n}^{\mathrm{s}}, \mathbf{F}_{\leq n}^{\mathrm{s}}, \mathbf{G}_{\leq 0}^{\mathrm{s}}, \mathbf{G}_{<n}^{\mathrm{s}}, \mathbf{G}_{\leq n}^{\mathrm{s}}\right\}$, and $\mathcal{S}_{\alpha \mathbf{O p} \beta}$ is a complete encoding of $\alpha \mathbf{O p} \beta$ for each $\mathbf{O p} \in\left\{\wedge, \vee, \mathbf{U}^{\mathrm{s}}, \mathbf{U}_{\geq n}^{\mathrm{s}}, \mathbf{U}_{>n}^{\mathrm{s}}, \mathbf{R}^{\mathrm{s}}, \mathbf{R}_{\geq n}^{\mathrm{s}}, \mathbf{R}_{>n}^{\mathrm{s}}\right\}$.

Recall, that an encoding $\mathcal{S}_{\phi}$ is complete if for every $\phi$ fine trace $\sigma=\left\langle I_{0}, v_{0}\right\rangle\left\langle I_{1}, v_{1}\right\rangle\left\langle I_{2}, v_{2}\right\rangle \ldots$ in $\operatorname{traces}(\mathcal{S})$, there is a run $\tau=s_{0} s_{1} s_{2} \ldots$ in $\mathcal{S}_{\phi}$ such that $\operatorname{trace}(\tau)=\sigma$ and for all points $(i, t)$ in $\sigma$ it holds that $\sigma^{(i, t)} \models \phi$ implies $v_{i}(\|\phi\|)=$ true. Thus, we can prove completeness by assuming a $\phi$-fine trace $\sigma \in \operatorname{traces}(\mathcal{S})$, extending it to a run $\tau$ by giving values for the auxiliary variables used in the encoding (setting $\|\phi\|$ to true exactly on those intervals where $\phi$ holds) and then arguing that all constraints of the encoding are satisfied. Lemma 5 is proven by structural induction. That is, it will assumed that the lemma holds for the subformulas $\alpha$ and $\beta$. Like Lemma 4, we will prove Lemma 5 separately for each operator.

As the lemma assume $\phi$-fineness, $\phi$ either holds at all points in a given interval in $\sigma$ or $\phi$ does not hold at any point inside the interval. We use the notation $\sigma^{(j, \cdot)} \models \phi$ to denote that $\phi$ is satisfied by all points belonging to interval $j$ and $\sigma^{(j, \cdot)} \not \models \phi$ to denote that no point inside the interval satisfies $\phi$.

Proof: For $\mathbf{O p}=\mathbf{U}^{\mathrm{s}}$. Auxiliary variable rules: Let $i \in \mathbb{N}$. As always, we set $s_{i}\left(\left\|\alpha \mathbf{U}^{\mathrm{s}} \beta\right\|\right) / s_{i}(\|\alpha\|) / s_{i}(\|\beta\|)$ to true iff $\sigma^{(i, \cdot)} \models \alpha \mathbf{U}^{\mathrm{s}} \beta / \sigma^{(i, \cdot)}=\alpha / \sigma^{(i, \cdot)} \models \beta$, respectively.

The transition constraints are satisfied: Let $i \in \mathbb{N}$. Now

- If $s_{i}\left(\left\|\alpha \mathbf{U}^{\mathrm{s}} \beta\right\|\right)=$ false then both Constraints 1 and 2 trivially hold at interval $i$.

- If $s_{i}\left(\left\|\alpha \mathbf{U}^{\mathrm{s}} \beta\right\|\right)=$ true and $I_{i}$ is open, then Constraint 2 is trivially satisfied. Furthermore, as $s_{i}\left(\left\|\alpha \mathbf{U}^{\mathrm{s}} \beta\right\|\right)=$ true we know that $\sigma^{(i, \cdot)} \models \alpha \mathbf{U}^{\mathrm{s}} \beta$. Pick any $t_{1} \in I_{i}$. By the semantics of $\mathbf{U}^{\mathrm{s}}$ we now know that there is a future time point $\left(j, t_{2}\right) \in T_{+}(\sigma,(i, t))$ such that $s_{j}(\|\beta\|)=$ true and $\|\alpha\|$ holds anywhere in between $\left(i, t_{1}\right)$ and $\left(j, t_{2}\right)$. Note that due to the fact that $I_{i}$ is open, there is a guarantee that interval $i$ contains time points lying in between $\left(i, t_{1}\right)$ and $\left(j, t_{2}\right)$, implying that $s_{i}(\|\alpha\|)=$ true. Now if $j=i$ or $j=i+1$, then Constraint 1 is satisfied. Furthermore, if $j>i+1$, then also $s_{i+1}(\|\alpha\|)=$ true and $\sigma^{(i+1, \cdot)} \models \alpha \mathbf{U}^{\mathrm{s}} \beta$. Thus, $s_{i+1}\left(\left\|\alpha \mathbf{U}^{\mathrm{s}} \beta\right\|\right)=$ true, implying that Constraint 2 is satisfied in this case as well.

- If $s_{i}\left(\left\|\alpha \mathbf{U}^{\mathrm{s}} \beta\right\|\right)=$ true and $I_{i}$ is a singleton, then Constraint 1 is trivially satisfied. Let $\left\{t_{1}\right\}=I_{i}$. Again, we pick a time point $\left(j, t_{2}\right) \in T_{+}\left(\sigma,\left(i, t_{2}\right)\right)$ such that $s_{j}(\|\beta\|)=$ true and $\|\alpha\|$ holds anywhere in between $\left(i, t_{1}\right)$ and $(j, t)$. Now if $j=i+1$ and $I_{j}$ is a singleton, then Constraint 2 is satisfied. If $j=i+1$ and $I_{j}$ is open, then $s_{j}(\|\alpha\|)=s_{j}(\|\beta\|)=$ true meaning that $\sigma^{(j, \cdot)}=\alpha \mathbf{U}^{\mathrm{s}} \beta$. Then $s_{j}\left(\left\|\alpha \mathbf{U}^{\mathrm{s}} \beta\right\|\right)=$ true and Constraint 2 is satisfied. If $j>i+1$ then we observe that $s_{i+1}(\|\alpha\|)=$ true and $\sigma^{(i+1, \cdot)} \models \alpha \mathbf{U}^{\mathrm{s}} \beta$, meaning that $s_{i+1}\left(\left\|\alpha \mathbf{U}^{\mathrm{s}} \beta\right\|\right)=$ true and Constraint 2 is satisfied in this case as well.

The fairness condition is satisfied: It is easy to see that now the fairness constraint $\mathcal{F}_{\alpha \mathbf{U}^{\mathrm{s}} \beta}$ holds as well. We set $\left\|\alpha \mathbf{U}^{\mathrm{s}} \beta\right\|$ to true precisely on those intervals on which $\alpha \mathbf{U}^{\mathrm{s}} \beta$ holds, implying that there is a future point at which $\beta$ holds. Thus, if $\left\|\alpha \mathbf{U}^{\mathrm{s}} \beta\right\|$ holds on all intervals starting from some point, then $\alpha \mathbf{U}^{\mathrm{s}} \beta$ holds globally starting from that point. Hence, there is always a future interval at which $\beta$ holds, meaning that $\beta$ (and thus $\|\beta\|$ ) holds infinitely often.

Proof: For $\mathbf{O p}=\mathbf{F}_{\leq 0}^{\mathrm{s}}$. Auxiliary variable rules: Let $i \in \mathbb{N}$. As always, we set $\left.s_{i}\left(\| \mathbf{F}_{\leq 0}^{\mathrm{s}} \alpha\right] \mid\right) / s_{i}(\|\alpha\|)$ to true iff $\sigma^{(i, \cdot)}=\mathbf{F}_{\leq 0}^{\mathrm{s}} \alpha / \sigma^{(i, \cdot)} \models \alpha$, respectively.

The transition constraints are satisfied: Let $i \in \mathbb{N}$. If $s_{i}\left(\left|\left[\mathbf{F}_{\leq 0}^{\mathrm{s}} \alpha\right]\right|\right)=$ false, then Constraint 3 is trivially satisfied. If, in contrast, $s_{i}\left(\left|\left[\mathbf{F}_{\leq 0}^{\mathrm{s}} \alpha\right]\right|\right)=$ true, then we know that $\sigma^{(i, \cdot)} \models \mathbf{F}_{\leq 0}^{\mathrm{s}} \alpha$. Thus, there is a $j>i$ such that $s_{j}(\|\alpha\|)=$ true and for every point in $I_{i+1}$ there is a point in $I_{j}$ that is at most 0 time units away. The latter implies that intervals $I_{i}, \ldots, I_{j}$ are all singletons. Thus, in particular, $I_{i}$ and $I_{i+1}$ are singletons. Furthermore, if $j=i+1$, then $s_{i+1}(\|\alpha\|)=$ true, implying that Constraint 3 is satisfied at interval $i$. If, in contrast $j>i+1$ then $\sigma^{(i+1, \cdot)} \models \mathbf{F}_{<0}^{\mathrm{s}} \alpha$ as well, implying that $s_{i+1}\left(\left|\left[\mathbf{F}_{<0}^{\mathrm{s}} \alpha\right]\right|\right)=$ true and, ultimately, that Constraint 3 is satisfied at interval $i$ also in this case.

Proof: For $\mathbf{O p}=\mathbf{F}_{<n}^{\mathrm{s}}$. Auxiliary variable rules: Let $i \in \mathbb{N}$. As always, we set $s_{i}\left(\left\|\mathbf{F}_{<n}^{\mathrm{s}} \alpha\right\|\right) / s_{i}(\|\alpha\|)$ to true iff $\sigma^{(i, \cdot)} \models \mathbf{F}_{<n}^{\mathrm{s}} \alpha / \sigma^{(i, \cdot)} \models \alpha$, respectively. Furthermore, we set $s_{0}(c)=0$ and $s_{0}$ (lefto) $=$ false. For $i>0$ we set $s_{i}(c)$ and $s_{i}($ lefto $)$ according to Constraints 7 and 8 and $s_{i-1}$.

The initial constraint is satisfied: Initial constraint $\mathcal{I}_{\mathbf{F}^{\mathrm{s}}{ }_{<n} \alpha}$ is trivially satisfied as $s_{0}(c)=0$ and $s_{0}($ lefto $)=$ false.

The transition constraints are satisfied: Let $i \in \mathbb{N}$. Let $\bar{i}=i$ if $I_{i}$ is open and $\bar{i}=i+1$ if $I_{i}$ is a singleton. Now we will show that interval $i$ satisfies Constraints 4 and 5 using a case distinction. 
- If $\left.s_{i}\left(\| \mathbf{F}_{<n}^{\mathrm{s}} \alpha\right]\right)=$ false or $s_{i+1}\left(\left\|\mathbf{F}_{<n}^{\mathrm{s}} \alpha\right\|\right)=$ true then Constraints 4 and 5 are trivially satisfied.

- If $\left.s_{i}\left(\| \mathbf{F}_{<n}^{\mathrm{s}} \alpha\right]\right)=$ true and $\left.s_{i+1}\left(\| \mathbf{F}_{<n}^{\mathrm{s}} \alpha\right]\right)=$ false, then we know that $\sigma^{(i, \cdot)}=\mathbf{F}_{<n}^{\mathrm{s}} \alpha$ and $\sigma^{(i+1, \cdot)} \not=$ $\mathbf{F}_{<n}^{\mathrm{s}} \alpha$. Now

- If $I_{i}$ is open then Constraint 5 is trivially satisfied. Furthermore, by semantics of $\mathbf{F}_{<n}^{\mathrm{s}}$ we know that $\sigma^{(i, \cdot)}=\mathbf{F}_{<n}^{\mathrm{s}} \alpha$ and $\sigma^{(i+1, \cdot)} \not \models \mathbf{F}_{<n}^{\mathrm{s}} \alpha$ implies that $\left.s_{i}(\| \alpha]\right)=$ true or $\left.s_{i}(\| \alpha]\right)=$ true. In either case, Constraint 4 is satisfied.

- If $I_{i}$ is a singleton then Constraint 4 is trivially satisfied. Furthermore, by semantics of $\mathbf{F}_{<n}^{\mathrm{s}}$ we know that $\sigma^{(i, \cdot)}=\mathbf{F}_{<n}^{\mathrm{s}} \alpha$ and $\sigma^{(i+1, \cdot)} \not \neq \mathbf{F}_{<n}^{\mathrm{s}} \alpha$ implies that (i) $I_{i+1}$ is a singleton as well and (ii) $s_{i+1}(\|\alpha\|)=$ true. Thus, Constraint 5 is satisfied as well.

It remains to be shown that Constraint 9 holds on interval $i$, which will be done by contradiction. Assume, Constraint 9 does not hold on interval $i$. Then, $T_{c}$ does not hold on interval $\left.i, s_{i}\left(\| \mathbf{F}_{<n}^{\mathrm{s}} \alpha\right]\right)=$ true and $I_{i}$ is a singleton or $s_{i}(\|\alpha\|)=$ false. As $\left.s_{i}\left(\| \mathbf{F}_{<n}^{\mathrm{s}} \alpha\right] \|\right)=$ true, we know that $\sigma^{(i, \cdot)}=\mathbf{F}_{<n}^{\mathrm{s}} \alpha$. Pick $j \leq i$ as large as possible such that $R_{c}$ holds at interval $j-1$ is such $j$ exists and set $j=0$ otherwise. Now we know that $s_{j}(c)=0$ and $s_{j}($ lefto $)=$ true iff $I_{j}$ is open.

- If $j=i$, the we know that the value of $s_{i}(c)=0$. Now

- If $I_{i}$ is a singleton, then the value of $\delta$ at interval $i$ is 0 as well, which implies that $T_{c}$ contrary to our assumption satisfied on interval $i$.

- If $I_{i}$ is open, then our assumption that Constraint 9 does not hold implies that $s_{i}(\|\alpha\|)=$ false. As $\sigma^{(i, \cdot)}=\mathbf{F}_{<n}^{\mathrm{s}} \alpha$, we now know that $\delta$ at interval $i$ can be at most $n$, implying that $T_{c}$ is satisfied and contradicting our assumption that Constraint 9 does not hold

- If $j<i$, then we know by the fact that $R_{c}$ does not hold on intervals $j, \ldots, i-1$ that the value of $c$ at intervals $j+1, \ldots, i$ has been set according to Constraint 8 . Hence, $s_{i}(c)$ is the difference between the left bound of $I_{i}$ and the left bound of $I_{j}$. Furthermore, $s_{i}(c)+s_{i}(\delta)$ is the difference between the right bound of $I_{i}$ and the left bound of $I_{j}$ and $s_{i}$ (lefto) $=$ true iff $I_{j}$ is open. By the fact that $R_{c}$ does not hold on intervals $j, \ldots, i-1$ and the fact that $s_{i}\left(\left\|\mathbf{F}_{<n}^{\mathrm{s}} \alpha\right\|\right)=$ true we know that $s_{j}\left(\left\|\mathbf{F}_{<n}^{\mathrm{s}} \alpha\right\|\right)=\ldots=s_{i}\left(\left\|\mathbf{F}_{<n}^{\mathrm{s}} \alpha\right\|\right)=$ true. Let $\bar{j}=j$ if $I_{j}$ is open and $\bar{j}=j+1$ if $I_{j}$ is a singleton. As $R_{c}$ does not hold on intervals $j, \ldots, i-1$ and $s_{j}\left(\left\|\mathbf{F}_{<n}^{\mathrm{s}} \alpha\right\|\right)=\ldots=s_{i}\left(\left\|\mathbf{F}_{<n}^{\mathrm{s}} \alpha\right\|\right)=$ true we know that $\left.s_{\bar{j}}(\| \alpha]\right)=\ldots=s_{i}(\|\alpha\|)=$ false. As $s_{j}\left(\left\|\mathbf{F}_{<n}^{\mathrm{s}} \alpha\right\|\right)=$ true, we know that $\sigma^{(j, \cdot)}=\mathbf{F}_{<n}^{\mathrm{s}} \alpha$. Thus, for each point in $I_{j}$ there is a future point at which $\alpha$ holds and that is less than $n$ time units away. As $s_{\bar{j}}(\|\alpha\|)=\ldots=s_{i}(\|\alpha\|)=$ false, this implies that every point in $I_{j}$ is less than $n$ time units away from a time point in $I_{i+1}$. Thus, the difference between the right bound of $I_{i}$ and the left bound of $I_{j}$ is less than $n$ time units if $I_{j}$ is a singleton and less than or equal to $n$ time units if $I_{j}$ is open. Recalling that the value of $c+\delta$ at interval $i$ is precisely said difference and $s_{i}($ lefto $)=$ true if $I_{j}$ is open, we conclude that $T_{c}$ is satisfied at interval $i$, contradicting our assumption.

In each case, we were able to show that the assumption that Constraint 9 does not hold leads to a contradiction.

Proof: For $\mathbf{O p}=\mathbf{F}_{\leq n}^{\mathrm{s}}$. The only difference between the proof for $\mathbf{F}_{\leq n}^{\mathrm{s}}$ and the proof for $\mathbf{F}_{<n}^{\mathrm{s}}$ is in arguing that Constraint 9 is satisfied.

- The case where $i=j$ and $I_{i}$ is a singleton does not need modification.

- In the case where $i=j$ and $I_{i}$ is open, we observe that $\sigma^{(i, \cdot)} \models \mathbf{F}_{\leq n}^{\mathrm{s}} \alpha$ and $s_{i}(\|\alpha\|)=$ false. Thus, we know that the value of $\delta$ at interval $i$ is at most $n$. Furthermore, as $I_{i}$ is open, we know that $s_{i}($ lefto $)=$ true, contradicting the assumption that $T_{c}$ is not satisfied.

- In the case where $j<i$, we observe that $s_{\bar{j}}(\|\alpha\|)=$ $\ldots=s_{i}(\|\alpha\|)=$ false and $\sigma^{(j, \cdot)}=\mathbf{F}_{<n}^{\mathrm{s}} \alpha$. Thus, the difference between the left bound of $\bar{I}_{j}$ and the right bound of $I_{i}$ is less than or equal to $n$ and less than $n$ if $I_{j}$ is a singleton and $I_{i+1}$ is open, again leading to a contradiction based on the fact that $T_{c}$ is satisfied.

Proof: For $\mathbf{O p}=\mathbf{U}_{>n}^{\mathrm{s}}$. Auxiliary variable rules: Let $i \in \mathbb{N}$. As always, we set $s_{i}\left(\left\|\alpha \mathbf{U}_{>n}^{\mathrm{s}} \beta\right\|\right) / s_{i}(\|\alpha\|)$ / $s_{i}(\|\beta\|)$ to true iff $\sigma^{(i, \cdot)} \models \alpha \mathbf{U}_{>n}^{\mathrm{s}} \beta / \sigma^{(i, \cdot)} \models \alpha$ / $\sigma^{(i, \cdot)} \mid=\beta$, respectively. We set $s_{0}(c)=0$ and $s_{0}($ righto $)=$ false. For $i>0$ we set $s_{i}(c)$ and $s_{i}$ (righto) according to Constraints 14 and 15 and $s_{i-1}$. Furthermore, we set $s_{i}($ oblig $)=$ true iff at least one of the following cases holds:

1) $\sigma^{(i, \cdot)} \models \alpha \mathbf{U}_{>n}^{\mathrm{s}} \beta$

2) $n>0, i>0$ and $\sigma^{(i-1, \cdot)}=\alpha \mathbf{U}_{>n}^{\mathrm{s}} \beta$,

3) $i>0, I_{i-1}$ is open, $s_{i-1}$ (oblig) $=$ true and $\hat{r}$ neither holds on interval $i-1$ nor on interval $i$.

4) $i>0, I_{i-1}$ is a singleton, $s_{i-1}($ oblig $)=$ true and $\hat{r}$ does not hold on interval $i$ or $I_{i}$ is open.

The transition constraints are satisfied: Let $i \in \mathbb{N}$. Note, that the rules for setting the value of oblig ensure that Constraint 10 is satisfied on interval $i$.

Assume $\sigma^{(i, \cdot)} \models \alpha \mathbf{U}_{>n}^{\mathrm{s}} \beta$ and $n>0$. Take $t \in I_{i}$ such that the difference between the right bound of $I_{i}$ and $t$ is less than $n$. Now there is a future time point more than $n$ time units from $(i, t)$ at which $\beta$ holds and up to which $\alpha$ holds. The fact that this point is more than $n$ time units away implies that either $I_{i+1}$ is open or the time point is on interval $i+2$ or a later interval. In either case $s_{i+1}(\|\alpha\|)=$ true. Together with the rules for setting the value of oblig, this implies that Constraint 11 is satisfied. 
If, in contrast, $\sigma^{(i, \cdot)} \not \models \alpha \mathbf{U}_{>n}^{\mathrm{s}} \beta$ then Constraint 11 is trivially satisfied and if $n=0$, Constraint 11 is not used at all.

Next, we show that Constraints 12 and 13 are satisfied on interval $i$.

- Assume $s_{i}($ oblig $)=$ false. In this case, Constraints 12 and 13 are trivially satisfied.

- Assume $s_{i}($ oblig $)=s_{i}\left(\left\|\alpha \mathbf{U}_{>n}^{\mathrm{s}} \beta\right\|\right)=$ true. Now if $I_{i}$ is open, then $s_{i}(\|\alpha\|)=$ true. Furthermore, by Constraint 14 we know $s_{i+1}(c)=0$ and $s_{i+1}($ righto $)=$ true iff $I_{i}$ is open. Now

- If $s_{i+1}($ oblig $)=s_{i+1}(\|\alpha\|)=$ true, then Constraints 12 and 13 are trivially satisfied.

- If $\left.s_{i+1}(\| \alpha]\right)=$ false, then the fact that $\sigma^{(i, \cdot)} \models$ $\alpha \mathbf{U}_{>n}^{\mathrm{s}} \beta$ implies that $n=0$ and one of the following:

* $s_{i}(\|\beta\|)=$ true and $I_{i}$ is open. In this case, $s_{i}(\delta)>0$, meaning that $T_{c}$ and $\hat{r}$ are satisfied on interval $i$ and ultimately that Constraints 12 and 13 are satisfied.

* $s_{i+1}(\|\beta\|)=$ true, $I_{i}$ is open and $I_{i+1}$ is a singleton. In this case, $s_{i+1}$ (righto $)=$ true, implying that $T_{c}$ and $\hat{r}$ are satisfied on interval $i+1$ and ultimately that Constraints 12 and 13 are satisfied.

- If $s_{i+1}($ oblig $)=$ false then by the fact that $s_{i}\left(\left\|\alpha \mathbf{U}_{>n}^{\mathrm{s}} \beta\right\|\right)=$ true and Rule 2 for setting the value of oblig we know that $n=0$. If we now assume that $I_{i}$ is a singleton, then by Rule 4 for setting the value of oblig we know that $\hat{r}$ holds on interval $i+1$ and $I_{i+1}$ is a singleton. The latter, however, implies that $s_{i+1}(c)+s_{i+1}(\delta)=0$ and, thus, that $\hat{r}$ does not hold on interval $i+1$. Thus, assuming $I_{i}$ to be a singleton leads to a contradiction and we know that $I_{i}$ is open.

Now $I_{i+1}$ is a singleton and we know that $s_{i+1}(c)+s_{i+1}(\delta)=0$ and $\hat{r}$ is not satisfied on interval $i+1$. Now Rule 3 for setting the value of oblig and the fact that $s_{i+1}(o b l i g)=$ false imply that $\hat{r}$ holds on interval $i$. As $I_{i}$ is open and $\sigma^{(i, \cdot)} \models \alpha \mathbf{U}_{>n}^{\mathrm{s}} \beta$, now $s_{i}(\|\alpha\|)=$ true and Constraints 12 and 13 are satisfied.

- Assume $s_{i}($ oblig $)=$ true and $s_{i}\left(\left\|\alpha \mathbf{U}_{>n}^{\mathrm{s}} \beta\right\|\right)=$ false. Choose $j<i$ as large as possible such that $s_{j}\left(\left\|\alpha \mathbf{U}_{>n}^{\mathrm{s}} \beta\right\|\right)=$ true. We know that a corresponding interval exists based on the fact that $s_{i}($ oblig $)=$ true. Let $\bar{j}=j$ if $I_{j}$ is open and $\bar{j}=j+1$ otherwise.

Now by the choice of $j$ we know $s_{j}($ oblig $)=\ldots=$ $s_{i}($ oblig $)=$ true.

- Assume $n>0$. Based on the rules for setting the value of oblig, we now know that $\hat{r}$ does not hold on intervals $j+2, \ldots, i$ and not on $j+1$ either if $I_{j+1}$ is open. Furthermore, if $I_{j+1}$ is a singleton as $s_{j+1}(c)=0$ we know that $s_{j+1}(c)+s_{j+1}(\delta)=0$ meaning $T_{c}$ (and, thus, $\hat{r}$ ) does not hold on interval $j+1$. Thus, $\hat{r}$ does not hold on intervals $j+1, \ldots, i$.

- Assume $n=0$. Based on the rules for setting the value for oblig, we now immediately know that $\hat{r}$ does not hold on intervals $j+1, \ldots, i$ and not on interval $j$ either if $I_{j}$ is open.

Now as $\sigma^{(j,)} \models \alpha \mathbf{U}_{>n}^{\mathrm{s}} \beta$, we can pick $k \geq j$ such that $s_{k}(\|\beta\|)=$ true, for every point in $I_{j}$ there is a point in $I_{k}$ that is more than $n$ time units away and $s_{\bar{j}}(\|\alpha\|)=\ldots=s_{\bar{k}}(\|\alpha\|)=$ true with $\bar{k}=k$ if $I_{j}$ is open and $\bar{k}=k-1$ if $I_{k}$ is a singleton. We observe that $k=j$ is possible only if $n=0$ and $I_{j}$ is open. In this case, however, $T_{c}$ and $\|\beta\|$ would both hold on interval $j$, meaning that $\hat{r}$ holds and contradicting our previous observation that $\hat{r}$ does not hold on interval $j$ if $I_{j}$ is open and $n=0$. Thus, $k>j$.

Take an arbitrary $m \in \mathbb{N}$ with $j<m \leq i$. Now we note that $s_{m}($ righto $)=$ true iff $I_{j}$ is open. Furthermore, $s_{m}(c)$ is the difference between the left bound of $I_{m}$ interval and the right bound of $I_{j}$. Correspondingly, $s_{m}(c)+s_{m}(\delta)$ is the difference between the right bounds of $I_{m}$ and $I_{j}$. Now as $\hat{r}$ does not hold on interval $m$, we know that either $s_{m}(\|\beta\|)=$ false or $T_{c}$ does not hold. By the definition of $T_{c}$, the latter implies that the difference between the right bounds of $I_{m}$ and $I_{j}$ is less than or equal to $n$ and less than $n$ if $I_{j}$ is open, meaning that there is a point in $I_{j}$ for which there is no point in $I_{m}$ that is $>n$ time units away. This allows us to conclude that $k \neq m$. As we picked an $j<m \leq i$ this means that $k>i$ and, thus, $s_{i}(\|\alpha\|)=$ true.

- Now if $\hat{r}$ holds on interval $i+1$ and $I_{i}$ is open, then Constraints 12 and 13 are satisfied on interval $i$.

- If $\hat{r}$ holds on interval $i+1, I_{i}$ is a singleton and $I_{i+1}$ is a singleton, then Constraints 12 and 13 are satisfied on interval $i$.

- If $\hat{r}$ holds on interval $i+1, I_{i}$ is a singleton and $I_{i+1}$ is open, according to Rule 4 for setting the value of oblig we have $s_{i+1}($ oblig $)=$ true. Furthermore, as $k>i$ and $I_{i+1}$ is open we have $\bar{k} \geq i+1$, implying that $s_{i+1}(\|\alpha\|)=$ true. Thus, Constraints 12 and 13 are satisfied on interval $i$.

- If $\hat{r}$ does not hold on interval $i+1$, then we can use the same argument used to show that $k>i$ to show that, in fact, $k>i+1$, implying that $s_{i+1}(\|\alpha\|)=$ true. Furthermore, by the Rules 3 and 4 for setting the value of oblig and the fact that $\hat{r}$ does not hold on intervals $i$ and $i+1$, we also know that $s_{i+1}($ oblig $)=$ true. Hence, Constraints 12 and 13 are satisfied on interval $i$. 
The fairness condition is satisfied: It remains to be shown that the fairness constraint $\mathcal{F}_{\alpha \mathbf{U}_{>n}^{\mathrm{s}} \beta}$ is satisfied by our choice of values, which will be done by contradiction. Assume the fairness constraint is not satisfied. Then there is an $i \in \mathbb{N}$ such that $s_{i}$ (oblig) $=s_{i+1}($ oblig $)=$ $\ldots=$ true and $s_{i}(\|\beta\|)=s_{i+1}(\|\beta\|)=\ldots=$ false. As $s_{i}(\|\beta\|)=s_{i+1}(\|\beta\|)=\ldots=$ false, we know that intervals $i, i+1, \ldots$ do not satisfy $\alpha \mathbf{U}_{>n}^{\mathrm{s}} \beta$ and, thus, $\left.s_{i}\left(\| \alpha \mathbf{U}_{>n}^{\mathrm{s}} \beta\right]\right)=s_{i+1}\left(\left\|\alpha \mathbf{U}_{>n}^{\mathrm{s}} \beta\right\|\right)=\ldots=$ false. By the rules for setting the value of oblig, we set oblig to true only if $\left\|\alpha \mathbf{U}_{>n}^{\mathrm{s}} \beta\right\|$ hold on the current or the previous interval or oblig holds on the previous interval. Thus, the fact that $s_{i}($ oblig $)=$ true implies that there is an interval before interval $i$ on which $\alpha \mathbf{U}_{>n}^{\mathrm{s}} \beta$ holds.

Pick $j$ as large as possible such that $j<i$ and $s_{i}\left(\left\|\alpha \mathbf{U}_{>n}^{\mathrm{s}} \beta\right\|\right)=$ true. Let $\bar{j}=j$ if $I_{j}$ is open and $\bar{j}=j+1$ if $I_{j}$ is a singleton. As $\sigma^{(j, \cdot)} \models \alpha \mathbf{U}_{>n}^{\mathrm{s}} \beta$, there is a $k \geq j$ such that $s_{k}(\|\beta\|)=$ true, for every point in $I_{j}$ there is a point in $I_{k}$ that is more than $n$ time units away and $s_{\bar{j}}(\|\alpha\|)=\ldots=s_{\bar{k}}(\|\alpha\|)=$ true with $\bar{k}=k$ if $I_{j}$ is open and $\bar{k}=k-1$ if $I_{k}$ is a singleton. As $s_{i}(\|\beta\|)=s_{i+1}(\|\beta\|)=\ldots=$ false, we know that $k<i$. Recall, that we picked $j$ to be the last interval at which $\left\|\alpha \mathbf{U}_{>n}^{\mathrm{s}} \beta\right\|$ holds. Hence, we know that oblig on any later interval can only be set to true based on Rules 3 and 4. As both of these rules require oblig to hold on the respective previous interval we know that $s_{j}($ oblig $)=s_{j+1}($ oblig $)=\ldots=$ true.

- Assume that $k=j$. By the semantics of $\mathbf{U}_{>n}^{\mathrm{s}}$, this implies that $n=0$ and $I_{i}$ is open. Then $T_{c}$ is satisfied on interval $j$. As, additionally, $s_{k}(\|\beta\|)=$ true and, thus, $\hat{r}$ holds, the rules for setting the value of oblig imply that $s_{i+1}($ oblig $)=$ false, contradicting our observation that $s_{j}($ oblig $)=s_{j+1}($ oblig $)=\ldots=$ true.

- Assume that $k=j+1$ and $n>0$. Then $I_{j+1}$ is open and $s_{j+1}(\delta)>n$. Thus, both $T_{c}$ and $\|\beta\|$ hold on interval $j+1=k$, implying that $\hat{r}$ holds. As $I_{j+1}$ is open, our rules for setting the value for oblig now imply that $s_{j+2}($ oblig $)=$ false, contradicting our observation that $s_{j}($ oblig $)=s_{j+1}($ oblig $)=\ldots=$ true.

- Assume that $k>j+1$ or $k=j+1$ and $n=0$. Then oblig was set to true on interval $k$ by Rule 3 or 4 implying that $\hat{r}$ does not hold on interval $k$ or $I_{k}$ is open. As oblig is set to true on interval $k+1$ by Rule 3 or 4 as well, $I_{k}$ being open again implies that $\hat{r}$ does not hold on interval $k$. Thus, $\hat{r}$ does not hold on interval $k$ and $T_{c}$ does not hold on interval $k$, due to the fact that we picked $k$ so that $s_{k}(\|\beta\|)=$ true. By the fact that $I_{j}$ is the last interval on which $\left\|\alpha \mathbf{U}_{>n}^{\mathrm{s}} \beta\right\|$ holds, we know that $c$ and righto are set based on Constraint 14 on interval $j+1$ and based on Constraint 15 on all later intervals. This implies that at interval $k$, the value of $c+\delta$ is the difference between the right bounds of $I_{k}$ and $I_{j}$. Furthermore, $s_{k}($ righto $)=$ true iff $I_{j}$ is open. Now

- If $I_{j}$ is open, then by the fact that $T_{c}$ does not hold on interval $k$ we know that the difference between the right bounds of $I_{j}$ and $I_{k}$ is less than $n$. This, however, contradicts the fact that $k$ was chosen such that for every point in $I_{j}$ there is a point in $I_{k}$ that is more than $n$ time units away.

- If $I_{j}$ is a singleton, then the difference between the right bounds of $I_{j}$ and $I_{k}$ is less than or equal to $n$. Again, this contradicts the fact that for every point in $I_{j}$ there is a point in $I_{k}$ that is more than $n$ time units away.

Thus, assuming that the fairness constraint $\mathcal{F}_{\alpha \mathbf{U}_{>n}^{\mathrm{s}} \beta}$ is not satisfied leads to a contradiction.

Proof: For $\mathbf{O p}=\mathbf{U}_{\geq n}^{\mathrm{s}}$. To obtain the proof for $\mathbf{U}_{\geq n}^{\mathrm{s}}$, we make the following changes to the proof for $\mathbf{U}_{>n}^{\mathrm{s}}$.

- All cases in which $n=0$ are now contradictions, as we encode $\mathbf{U}_{\geq 0}^{\mathrm{s}}$ by the $\mathbf{U}^{\mathrm{s}}$ encoding.

- No substantial changes are needed to show that Constraints 10 and 11 are satisfied.

- When showing that Constraints 12 and 13 are satisfied:

- In the case where $s_{i}($ oblig $)=s_{i}\left(\left|\left[\alpha \mathbf{U}_{\geq n}^{\mathrm{s}} \beta\right]\right|\right)=$ true the only valid option is that $s_{i+1}($ oblig $)=$ $s_{i+1}(\|\alpha\|)=$ true, as all other cases required $n=$ 0 .

- In the case where $s_{i}$ (oblig) $=$ true and $s_{i}\left(\left|\left[\alpha \mathbf{U}_{\geq n}^{\mathrm{s}} \beta\right]\right|\right)=$ false, we pick $k$ so that for every point in $j$ there is a point at least $n$ time units away in $I_{k}$ (and the properties regarding $\| \alpha \rrbracket$ and $\|\beta\|$ hold).

After picking $m$, we note that if $T_{c}$ does not hold on interval $m$ then by the definition of $T_{c}$ the difference between the right bounds of $I_{m}$ and $I_{j}$ is less than or equal to $n$ and less than $n$ if $I_{j}$ is open or $I_{m}$ is a singleton. Then, there is a point in $I_{j}$ for which there is no point in $I_{m}$ that is $\geq n$ time units away. Again, we conclude that $k \neq m$. We proceed as before.

- When showing that the fairness constraint is satisfied we pick $k$ so that for every point in $j$ there is a point at least $n$ time units away in $I_{k}$ (and the properties regarding $\| \alpha \rrbracket$ and $\|\beta\|$ hold).

Then, the final case distinction is replaced by

- If $I_{j}$ is open or $I_{k}$ a singleton, then by the fact that $T_{c}$ does not hold on interval $k$ we know that the difference between the right bounds of $I_{j}$ and $I_{k}$ is less than $n$. This, however, contradicts the fact that $k$ was chosen such that for every point in $I_{j}$ there is a point in $I_{k}$ that is at least $n$ time units away.

- If $I_{j}$ is a singleton and $I_{k}$ an open interval, then the difference between the right bounds of $I_{j}$ and 
$I_{k}$ is less than or equal to $n$. Again, this contradicts the fact that for every point in $I_{j}$ there is a point in $I_{k}$ that is at least $n$ time units away.

Proof: For $\mathbf{O p}=\mathbf{R}^{\mathbf{s}}$. Auxiliary variable rules: Let $i \in \mathbb{N}$. As always, we set $s_{i}\left(\left\|\alpha \mathbf{R}^{\mathrm{s}} \beta\right\|\right) / s_{i}(\|\alpha\|) / s_{i}(\|\beta\|)$ to true iff $\sigma^{(i, \cdot)} \models \alpha \mathbf{R}^{\mathrm{s}} \beta / \sigma^{(i, \cdot)}=\alpha / \sigma^{(i, \cdot)} \models \beta$, respectively. We set $s_{i}$ (oblig $)=$ true iff at least one of the following holds:

1) $I_{i}$ is open and $s_{i}\left(\left\|\alpha \mathbf{R}^{\mathrm{s}} \beta\right\|\right)=$ true,

2) $I_{i-1}$ is a singleton and $s_{i-1}\left(\left\|\alpha \mathbf{R}^{\mathrm{s}} \beta\right\|\right)=$ true, or

3) $s_{i-1}($ oblig $)=$ true and $s_{i-1}(\|\alpha\|)=$ false.

The transition constraints are satisfied: Let $i \in \mathbb{N}$. Constraints 16,17 and 18 are trivially satisfied based on the rules for setting the value of oblig. Thus, it only remains to be shown that Constraint 19 is satisfied as well. We distinguish the following cases:

- Case 1: $s_{i}($ oblig $)=$ false. In this case, Constraint 19 is trivially satisfied.

- Case 2: $s_{i}($ oblig $)=$ true and Rule 1 for setting the value of oblig applies. That is, $I_{i}$ is open and $s_{i}\left(\left\|\alpha \mathbf{R}^{\mathrm{s}} \beta\right\|\right)=$ true. As $s_{i}\left(\left\|\alpha \mathbf{R}^{\mathrm{s}} \beta\right\|\right)=$ true, we know that $\sigma^{(i, \cdot)}=\alpha \mathbf{R}^{\mathrm{s}} \beta$. On any open interval satisfying $\alpha \mathbf{R}^{\mathrm{s}} \beta$ either $\alpha$ or $\beta$ (or both) must hold, immediately implying that Constraint 19 is satisfied.

- Case 3: $s_{i}$ (oblig) $=$ true and Rule 1 does not apply but Rule 2 does apply. Then $I_{i-1}$ is a singleton and $s_{i-1}\left(\left\|\alpha \mathbf{R}^{\mathbf{s}} \beta\right\|\right)=$ true, implying that $\sigma^{(i-1, \cdot)}=\alpha \mathbf{R}^{\mathrm{s}}$ $\beta$. If $I_{i}$ itself is a singleton, then there is no time point in between the single time point in $I_{i-1}$ and the single time point in $I_{i}$. Thus, $\sigma^{(i-1, \cdot)}=\alpha \mathbf{R}^{\mathrm{s}} \beta$ implies that $s_{i}(\|\beta\|)=$ true, in turn implying that Constraint 19 is satisfied. If, in contrast, $I_{i}$ is open, then $\sigma^{(i-1, \cdot)} \models$ $\alpha \mathbf{R}^{\mathbf{s}} \beta$ implies that either $s_{i}(\|\alpha\|)=$ true or $s_{i}(\|\beta\|)=$ true (or both). Thus, also in this case Constraint 19 is satisfied.

- Case 4: $s_{i}($ oblig $)=$ true and Rule 1 and Rule 2 do not apply. In this case, Rule 3 has to apply, as we would not have set $s_{i}($ oblig $)=$ true if no rule applied. Thus, $s_{i-1}($ oblig $)=$ true and $s_{i-1}(\|\alpha\|)=$ false. We now choose $j<i$ as large as possible such that (i) $s_{j}($ oblig) $=$ true and (ii) either $j=0$ or $s_{j-1}($ oblig $)=$ false. Now we know that $s_{j}($ oblig $)=$ true based on Rule 1 or Rule 2 . Let $\bar{j}=j$ iff Rule 1 applies to interval $j$ and $\bar{j}=j-1$ otherwise. We now know that $s_{\bar{j}}\left(\left\|\alpha \mathbf{R}^{\mathrm{s}} \beta\right\|\right)=$ true and, thus, $\sigma^{(\bar{j}, \cdot)} \models \alpha \mathbf{R}^{\mathrm{s}} \beta$. Furthermore, as oblig propagated up to interval $i$ through Rule 3, we know that $s_{j}(\|\alpha\|)=$ $\ldots=s_{i-1}(\|\alpha\|)=$ false. Now take any $t_{1} \in I_{i}$ and $t_{2} \in I_{j}$. We now perform another case distinction based on the type of $I_{i}$.

- If $I_{i}$ is a singleton, then for any $(k, t) \in T(\sigma)$ with $\left(\bar{j}, t_{2}\right) \prec(k, t) \prec\left(i, t_{1}\right)$ it holds that $j \leq k<i$, implying that $s_{k}(\|\alpha\|)=$ false. Thus, there is no time point in between $\left(\bar{j}, t_{2}\right)$ and $\left(i, t_{1}\right)$ at which $\alpha$ holds. As $\sigma^{\left(\bar{j}, t_{2}\right)} \models \alpha \mathbf{R}^{\mathrm{s}} \beta$, this means that $s_{i}(\|\beta\|)=$ true and Constraint 19 is satisfied.

- If $I_{i}$ is open, then for any $(k, t) \in T(\sigma)$ with $\left(\bar{j}, t_{2}\right) \prec(k, t) \prec\left(i, t_{1}\right)$ it holds that $j \leq k \leq i$, meaning that $s_{j}(\|\alpha\|)=$ true implies $k=i$. Thus, if there is a time point in between $\left(\bar{j}, t_{2}\right)$ and $\left(i, t_{1}\right)$ at which $\alpha$ holds, that time point has to be part of interval $i$, meaning that $s_{i}(\|\alpha\|)=$ true. If there is no such time point, then the fact that $\sigma^{\left(\bar{j}, t_{2}\right)} \models$ $\alpha \mathbf{R}^{\mathrm{s}} \beta$ implies that $s_{i}(\|\beta\|)=$ true. Hence, we have $s_{i}(\|\alpha\|)=$ true or $s_{i}(\|\beta\|)=$ true (or both) and Constraint 19 is satisfied.

Either way, we were able to demonstrate that Constraint 19 is satisfied.

Proof: For $\mathbf{O p}=\mathbf{G}_{\leq 0}^{\mathrm{s}}$. Auxiliary variable rules: Let $i \in \mathbb{N}$. As always, we set $\left.s_{i}\left(\| \mathbf{G}_{\leq 0}^{\mathrm{s}} \alpha\right] \mid\right) / s_{i}(\|\alpha\|)$ to true iff $\sigma^{(i, \cdot)} \models \mathbf{G}_{\leq 0}^{\mathrm{s}} \alpha / \sigma^{(i, \cdot)} \models \alpha$, respectively.

The transition constraints are satisfied: Let $i \in \mathbb{N}$. Now

- If $s_{i}\left(\left|\left[\mathbf{G}_{<0}^{\mathrm{s}} \alpha\right]\right|\right)=$ false or $I_{i}$ is open or $I_{i+1}$ is open, then Constraint 20 is trivially satisfied on interval $i$.

- If, $\left.s_{i}\left(\| \mathbf{G}_{<0}^{\mathrm{s}} \alpha\right] \|\right)=$ true and $I_{i}$ is a singleton and $I_{i+1}$ is a singleton, then pick $j \geq i+1$ as large as possible such that $I_{i}, \ldots, I_{j}$ are all singletons. $s_{i}\left(\left[\mathbf{G}_{\leq 0}^{\mathrm{s}} \alpha\right] \mid\right)=$ true means that $\sigma^{(i, \cdot)}=\mathbf{G}_{<0}^{\mathrm{s}} \alpha$. Thus, we know that $s_{i+1}(\|\alpha\|)=\ldots=s_{j}(\|\alpha\|)=$ true. This implies that $\sigma^{(i+1, \cdot)} \models\left|\left[\mathbf{G}_{\leq 0}^{\mathrm{s}} \alpha\right]\right|$ as well and, thus, $s_{i+1}\left(\left|\left[\mathbf{G}_{\leq 0}^{\mathrm{s}} \alpha\right]\right|\right)=$ true. Thus, Constraint 20 is satisfied on interval $i$ in this case as well.

Hence, Constraint 20 is in each case satisfied on interval $i$.

Proof: For $\mathbf{O p}=\mathbf{G}_{<n}^{\mathbf{S}}$. Auxiliary variable rules: Let $i \in \mathbb{N}$. As always, we set $s_{i}\left(\left\|\mathbf{G}^{\mathrm{s}}<n \alpha\right\|\right) / s_{i}(\|\alpha\|)$ to true iff $\sigma^{(i, \cdot)}=\mathbf{G}_{<n}^{\mathrm{s}} \alpha / \sigma^{(i, \cdot)} \models \alpha$, respectively. We set $s_{0}(c)=$ $n+1$ and $s_{0}($ righto $)=$ true. For $i>0$ we set $s_{i}(c)$ and $s_{i}$ (righto) according to Constraints 22 and 23 and $s_{i-1}$.

The transition constraints are satisfied: Let $i \in \mathbb{N}$.

It remains to show that Constraint 21 is satisfied on interval $i$.

- Case 1: $T_{c}$ does not hold on interval $i$ and $s_{i}\left(\left\|\mathbf{G}_{<n}^{\mathrm{s}} \alpha\right\|\right)=$ false or $I_{i}$ is a singleton. In this case Constraint 21 is trivially satisfied.

- Case 2: $s_{i}\left(\| \mathbf{G}_{<n}^{\mathrm{s}} \alpha \rrbracket\right)=$ true an $I_{i}$ is open. As $\sigma^{(i, \cdot)} \models$ $\mathbf{G}^{\mathrm{s}}{ }_{<n} \alpha$ and $I_{i}$ is open, we now know that $s_{i}(\|\alpha\|)=$ true. Thus, Constraint 21 is satisfied.

- Case 3: $T_{c}$ holds on interval $i$. Then we note that there is a previous interval on which $\left\|\mathbf{G}^{\mathrm{S}}{ }_{<n} \alpha\right\|$ holds. If no such previous interval would exist, then $s_{i}(c)$ would be at least the initial value of $n+1$, contradicting the assumption that $T_{c}$ holds. We can, thus, pick $j<i$ is as large as possible such that $\left.s_{j}\left(\| \mathbf{G}^{\mathbf{S}}<n \alpha\right]\right)=$ true, implying $\sigma^{(j, \cdot)} \models \mathbf{G}_{<n}^{\mathrm{s}} \alpha$. 
As is easy to see, repeated application of Constraints 22 and 23 leads to $s_{i}(c)$ being the difference between the left bound of $I_{i}$ and the right bound of $I_{j}$. Furthermore, $s_{i}($ righto $)=$ true iff $I_{j}$ is open. As $T_{c}$ holds at interval $i$ we know that $s_{i}(c)<n$. That is, the difference between left bound of $I_{i}$ and the right bound of $I_{j}$ is less than $n$ time units. Indiscriminately of whether $I_{j}$ and $I_{i}$ are open or singletons, this implies that there are $t_{1} \in I_{i}$ and $t_{2} \in I_{j}$ with $t_{1}-t_{2}<n$. This implies that $s_{i}(\|\alpha\|)=$ true, as $\sigma^{(j, \cdot)} \models \mathbf{G}_{<n}^{\mathrm{s}} \alpha$. Thus, Constraint 21 is satisfied in this case as well.

Proof: For $\mathbf{O p}=\mathbf{G}_{\leq n}^{\mathrm{s}}$. The proof for $\mathbf{O p}=\mathbf{G}^{\mathrm{s}}{ }_{\leq n}$ proceeds precisely as the proof for $\mathbf{O p}=\mathbf{G}_{<n}^{\mathrm{s}}$ up to the point in Case 2 where we observe that $s_{i}(c)<n$. For $\mathbf{O p}=$ $\mathbf{G}_{\leq n}^{\mathrm{s}}$, we observe instead that $s_{i}(c)<n$ or $s_{i}(c) \leq n$ and intervals $I_{i}$ and $I_{j}$ are both singletons. Thus, we can pick time points in $I_{i}$ and $I_{j}$ that are $\leq n$ time units apart. Hence, the fact that $\sigma^{(j, \cdot)} \models \mathbf{G}^{\mathrm{s}}{ }_{<n} \alpha$ again implies $s_{i}(\|\alpha\|)=$ true and Constraint 21 is satisfied.

Proof: For $\mathbf{O p}=\mathbf{R}_{>n}^{\mathrm{s}}$. Auxiliary variable rules: Let $i \in \mathbb{N}$. As always, we set $s_{i}\left(\left\|\alpha \mathbf{R}_{>n}^{\mathrm{s}} \beta\right\|\right) / s_{i}(\|\alpha\|) / s_{i}(\|\beta\|)$ to true iff $\sigma^{(i, \cdot)} \models \alpha \mathbf{R}_{>n}^{\mathrm{s}} \beta / \sigma^{(i, \cdot)} \models \alpha / \sigma^{(i, \cdot)} \models \beta$, respectively. We set $s_{0}(c)=0$ and $s_{0}($ lefto $)=$ false. For $i>0$ we set $s_{i}(c)$ and $s_{i}$ (lefto) according to Constraints 29, 30 and 31 and $s_{i-1}$. For setting the value of oblig, we use the same rules used for the untimed release $\mathbf{R}^{\mathrm{s}}$. That is, we set $s_{i}($ oblig $)=$ true iff at least one of the following holds:

1) $I_{i}$ is open and $s_{i}\left(\| \alpha \mathbf{R}_{>n}^{\mathrm{s}} \beta \rrbracket\right)=$ true,

2) $I_{i-1}$ is a singleton and $s_{i-1}\left(\left\|\alpha \mathbf{R}_{>n}^{\mathrm{s}} \beta\right\|\right)=$ true, or

3) $s_{i-1}($ oblig $)=$ true and $s_{i-1}(\|\alpha\|)=$ false.

The initial constraint is satisfied: Initial Constraint $\mathcal{I}_{\alpha \mathbf{R}_{>n}^{\mathrm{s}} \beta}$ is trivially satisfied as $s_{0}(c)=0$ and $s_{0}($ lefto $)=$ false.

The transition constraints are satisfied: Let $i \in \mathbb{N}$. We observe that Constraints 24, 25 and 26 correspond to Constraints 16, 17 and 18 in the encoding of the untimed release operator. Consequently, Constraints 24,25 and 26 are satisfied by the fact that we use the exact same rules for setting the value of $s_{i}(o b l i g)$.

The left hand side of the implication in Constraint 28 is satisfied precisely if $\sigma^{(i, \cdot)}=\alpha \mathbf{R}_{>n}^{\mathrm{s}} \beta$ and $I_{i}$ is an open interval whose bounds are more than $n$ time units apart. Then, there are time points $t_{1}, t_{2} \in I_{i}$ such that $t_{2}-t_{1}>n$. By the semantics of $\mathbf{R}^{\mathbf{s}}$, this implies that $\beta$ holds at $\left(i, t_{2}\right)$ or that $\alpha$ holds somewhere in between $\left(i, t_{1}\right)$ and $\left(i, t_{2}\right)$. Thus, $s_{i}(\|\alpha\|)=$ true or $s_{i}(\|\beta\|)=$ true and the right hand side of the implication is satisfied as well.

We now argue that Constraint 27 is satisfied on interval $i$ using a case distinction:

Case 1: $s_{i}($ oblig $)=$ false or $T_{c}$ is not satisfied at interval $i$ or interval $I_{i}$ is open and $s_{i}(\|\alpha\|)=$ true. In this case, Constraint 27 is trivially satisfied at interval $i$.
Case 2: $s_{i}($ oblig $)=$ true, either $I_{i}$ is a singleton or $s_{i}(\|\alpha\|)=$ false and $T_{c}$ is satisfied at interval $i$. Note that $T_{c}$ holding at interval $i$ implies that $s_{i}(c)>0$. Now pick $j \leq i$ as large as possible such that one of the following: (i) $j<i$ and $D_{c}$ holds at interval $j$, (ii) $R_{c}$ holds at interval $j-1$ or (iii) $j=0$. By the choice of $j$ we know that (a) $R_{c}$ does not hold at intervals $j \ldots i-1$ (b) $D_{c}$ does not hold at intervals $j+1, \ldots, i-1$ and (c) $s_{j}\left(\left\|\alpha \mathbf{R}_{>n}^{\mathrm{s}} \beta\right\|\right)=$ true. If $j$ was chosen based on (i) or (ii), this immediately follows from the definition of $D_{c}$ and $R_{c}$. If, in contrast, $j$ was chosen based on (iii) (implying $j=0$ ) then assuming $s_{j}\left(\left\|\alpha \mathbf{R}_{>n}^{\mathrm{s}} \beta\right\|\right)=$ false leads to the observation that both $\left\|\alpha \mathbf{R}_{>n}^{\mathrm{s}} \beta\right\|$ and oblig are false on a prefix of $\tau$. Thus $R_{c}$ holds on the first interval on whose successor $\left\|\alpha \mathbf{R}_{>n}^{\mathrm{s}} \beta\right\|$ holds. As $s_{i}\left(\left\|\alpha \mathbf{R}_{>n}^{\mathrm{s}} \beta\right\|\right)=$ true, this the case on is interval $i-1$ at the latest, contradicting the assumption that we picked $j$ based on (iii). Thus, $s_{j}\left(\left\|\alpha \mathbf{R}_{>n}^{\mathrm{s}} \beta\right\|\right)=$ true in each case.

Based on the update rules for $c$ and lefto, we know that $s_{i}($ lefto $)=$ true iff $I_{j}$ is open. Furthermore, $s_{i}(c)$ is the difference between the left bound of $I_{i}$ and the left bound of $I_{j}$, i.e. $s_{i}(c)+s_{i}(\delta)$ is the difference of the right bound of $I_{i}$ and the left bound of $I_{j}$. By our Case 2 assumption that $T_{c}$ is satisfied, this difference is greater than $n$. Therefore, we can indiscriminately of the type of $I_{i}$ and $I_{j}$ pick $t_{1} \in I_{i}, t_{2} \in I_{j}$ such that $t_{1}-t_{2}>n$.

Let $\bar{i}:=i$ if $I_{i}$ is open and $\bar{i}:=i-1$ otherwise. Furthermore, let $\bar{j}:=j$ if $I_{j}$ is open and $\bar{j}:=j+1$ otherwise. Note that $\bar{i} \geq \bar{j}$. (As $i>j$, we know that $\bar{i} \geq \bar{j}-1$. Additionally, $\bar{i}=\bar{j}-1$ would require that $i=j+1$ and that both $I_{i}$ and $I_{j}$ to be singletons which contradicts $c+\delta>n$ at interval $i$ ) Furthermore, note that the time points lying in between $\left(j, t_{1}\right)$ and $\left(i, t_{2}\right)$ now all belong to $I_{\bar{j}}, \ldots, I_{\bar{i}}$. We now claim that both $s_{\bar{j}}($ oblig $)=\ldots=s_{\bar{i}}($ oblig $)=$ true and $s_{\bar{j}}(\|\alpha\|)=\ldots=s_{\bar{i}}(\|\alpha\|)=$ false. Note, that by our Case 2 assumptions we have $s_{i}($ oblig $)=$ true and $s_{i}(\|\alpha\|)=$ false if $I_{i}$ is open (meaning $\bar{i}=i$ ). Thus, we only have to show that $s_{\bar{j}}($ oblig $)=\ldots=s_{i-1}($ oblig $)=$ true and $s_{\bar{j}}(\|\alpha\|)=\ldots=s_{i-1}(\|\alpha\|)=$ false, which will proven by induction over $k=\bar{j}, \ldots, i-1$, for each $k$ assuming that we established $s_{\bar{j}}($ oblig $)=\ldots=s_{k-1}($ oblig $)=$ true and $\left.s_{\bar{j}}(\| \alpha]\right)=\ldots=s_{k-1}(\|\alpha\|)=$ false already.

Base case: $k=\bar{j} \cdot s_{\bar{j}}($ oblig $)=$ true follows immediately from the fact that $s_{j}\left(\left\|\alpha \mathbf{R}_{>n}^{\mathrm{s}} \beta\right\|\right)=$ true and Rules 1 and 2 for setting the value of oblig.

Now assume $s_{k}(\|\alpha\|)=$ true. Recall, that $s_{i}($ oblig $)=$ true. This allows us to pick a $m$ as small as possible such that $k<m \leq i$ and $s_{m}($ oblig $)=$ true. Then:

- If $m=k+1$, then based on the fact that $s_{k}(\|\alpha\|)=$ true, we know that oblig was set to true on interval $m$ not based on Rule 3 but based on Rule 1 or 2 .

- If $m>k+1$, then by the choice of $m$ we know that $s_{m-1}($ oblig $)=$ false. Thus, we deduct that, again, oblig was not set to true on interval $m$ based on Rule 3 but based on Rule 1 or 2 . 
Now we split based on the rule by which oblig was set to true on interval $m$.

- If oblig was set to true based on Rule 1 and Rule 2 does not apply, then $s_{m}\left(\left\|\alpha \mathbf{R}_{>n}^{\mathrm{s}} \beta\right\|\right)=$ true and $s_{m-1}\left(\| \alpha \mathbf{R}_{>n}^{\mathrm{s}} \beta \rrbracket\right)=$ false (as otherwise Rule 2 would apply). Furthermore, $I_{m}$ is open, implying that $I_{m-1}$ is asingleton.

- If $m=k+1$, then as $s_{k}(\|\alpha\|)=$ true, $\left.s_{k}\left(\| \alpha \mathbf{R}_{>n}^{\mathrm{s}} \beta\right]\right)=$ false and $\left.s_{k+1}\left(\| \alpha \mathbf{R}_{>n}^{\mathrm{s}} \beta\right]\right)=$ true we conclude that $R_{c}$ holds at interval $m-1=$ $k>j$, contradicting observation (a).

- If $m>k+1$, then $s_{m-1}($ oblig $)=$ false. As additionally $s_{m}\left(\left\|\alpha \mathbf{R}_{>n}^{\mathrm{s}} \beta\right\|\right)=$ true, $\left.s_{k}\left(\| \alpha \mathbf{R}_{>n}^{\mathrm{s}} \beta\right] \|\right)=$ false, we know that $R_{c}$ holds on interval $m-1>k \geq j$, again contradicting observation (a).

- If oblig was set to true based on Rule 2, then $I_{m-1}$ is a singleton and $s_{m-1}\left(\| \alpha \mathbf{R}_{>n}^{\mathrm{s}} \beta \rrbracket\right)=$ true. Now:

- If $m=k+1$, then $I_{k}$ is a singleton, implying that $k>j$ (as $k \geq \bar{j}$ it is not possible that $k=j$ when $I_{k}$ is a singleton). Now $s_{k}(\| \alpha \rrbracket)=$ $s_{k}\left(\left\|\alpha \mathbf{R}_{>n}^{\mathrm{s}} \beta\right\|\right)=s_{k}($ oblig $)=$ true, $I_{k}$ is a singletonand $D_{c}$ holds at interval $k>j$. This contradicts observation (b).

- If $m=k+2$, we further split cases based on the type of $I_{k}$.

* If $I_{k}$ is open, then $s_{k}(\|\alpha\|)=$ true and $s_{k+1}\left(\left\|\alpha \mathbf{R}_{>n}^{\mathrm{s}} \beta\right\|\right)=$ true, meaning that $R_{c}$ is satisfied at interval $k$, again contradicting (a).

* If $I_{k}$ is a singleton, then we again observe that $k>j$. Now one last split is necessary:

- If $s_{k}\left(\left\|\alpha \mathbf{R}_{>n}^{\mathrm{s}} \beta\right\|\right)=$ true, then $D_{c}$ holds at interval $k>j$, contradicting (b).

- If $s_{k}\left(\left\|\alpha \mathbf{R}_{>n}^{\mathrm{s}} \beta\right\|\right)=$ false, then $R_{c}$ holds on interval $k$ based on the fact that, additionally, $s_{k}(\|\alpha\|)=$ true and $s_{k+1}\left(\left\|\alpha \mathbf{R}_{>n}^{\mathrm{s}} \beta\right\|\right)=$ true, contradicting (a).

- Assume $m>k+2$. By the choice of $m$ we know that $s_{m-2}($ oblig $)=$ false and $s_{m-1}($ oblig $)=$ false, implying that $s_{m-2}\left(\left\|\alpha \mathbf{R}_{>n}^{\mathrm{s}} \beta\right\|\right)=$ false Now $R_{c}$ holds at interval $m-2>k \geq j$ due to the fact that additionally $s_{m-1}\left(\left\|\alpha \mathbf{R}_{>n}^{\mathrm{s}} \beta\right\|\right)=$ true, again contradicting (a).

As each case ended in a contradiction we conclude that $s_{k}(\| \alpha \rrbracket)=$ false.

Inductive step: $\bar{j}<k<i$. Now $s_{k}($ oblig $)=$ true follows from the inductive hypothesis that $s_{k-1}($ oblig $)=$ true and $s_{k-1}(\|\alpha\|)=$ false and Rule 3 for setting the value of oblig. Furthermore, we can derive that $s_{k}(\|\alpha\|)=$ false by the same arguments used in the base case. Thus, we have shown inductively that $s_{\bar{j}}($ oblig $)=\ldots=s_{i-1}($ oblig $)=$ true and $s_{\bar{j}}(\|\alpha\|)=\ldots=s_{i-1}(\| \alpha \rrbracket)=$ false.
Now as $s_{\bar{j}}(\| \alpha \rrbracket)=\ldots=s_{i}(\|\alpha\|)=$ false we know that there is no time point in between $\left(j, t_{2}\right)$ and $\left(i, t_{1}\right)$ at which $\alpha$ holds. As $\sigma^{\left(j, t_{2}\right)} \models \alpha \mathbf{R}_{>n}^{\mathrm{s}} \beta$ and $t_{1}-t_{2}>n$ we can conclude that $\beta$ holds at time point $\left(i, t_{1}\right)$, meaning that $\left.s_{i}(\| \beta]\right)=$ true and ultimately implying that Constraint 27 is satisfied at interval $i$.

Proof: For $\mathbf{O p}=\mathbf{R}_{\geq n}^{\mathrm{s}}$. To adapt the proof for $\mathbf{O p}=$ $\mathbf{R}_{>n}^{\mathrm{s}}$ to $\mathbf{O p}=\mathbf{R}_{>n}^{\mathrm{s}}$ we only need to argue that in Case 2, based on the fact that $T_{c}$ holds we can pick $t_{1} \in I_{i}, t_{2} \in I_{j}$ with $t_{1}-t_{2} \geq n$. As $T_{c}$ holds, we know that the difference between the right bound of $I_{i}$ and the left bound of $I_{j}$ is $>n$ or the difference is $\geq n$ and both $I_{i}$ and $I_{j}$ are singletons. In both cases, we can pick $t_{1}, t_{2}$ with $t_{1}-t_{2} \geq n$.

\section{G. Proof of Lemma 6}

Lemma 6: Assume two states, $s$ and $t$, such that $s \approx t$. It holds that (i) $s \models \mathcal{I}$ iff $t=\mathcal{I}$, and (ii) $s \models \mathcal{I} \mathcal{N} \mathcal{V}$ iff $t \models \mathcal{I N} \mathcal{V}$. Furthermore, if there is a $\delta_{s} \in \mathbb{R}_{>0}$ and a state $s^{\prime}$ such that $s \cup\left\{\delta \mapsto \delta_{s}\right\} \cup\left\{y^{\prime} \mapsto s^{\prime}(y) \mid y \in X \cup Z\right\} \mid$ $\mathcal{T}$, then there is a $\delta_{t} \in \mathbb{R}_{\geq 0}$ and a state $t^{\prime}$ such that $t \cup\left\{\delta \mapsto \delta_{t}\right\} \cup\left\{y^{\prime} \mapsto t^{\prime}(y) \mid y \in X \cup Z\right\} \models \mathcal{T}$ and $s^{\prime} \approx t^{\prime}$.

Proof: As $s \approx t$ and the only atoms involving clock variables in $\mathcal{I}$ and $\mathcal{I} \mathcal{N} \mathcal{V}$ are of form $x \bowtie n$, the definitions of $\mathrm{m}_{x}$ and $\approx$ directly imply that (i) $s \models \mathcal{I}$ iff $t \models \mathcal{I}$, and (ii) $s=\mathcal{I N} \mathcal{V}$ iff $t \models \mathcal{I} \mathcal{N} \mathcal{V}$.

To prove the remaining claim, consider the state $s^{\prime \prime}$ such that (i) $s^{\prime \prime}(x)=s(x)+\delta_{s}$ for each clock $x \in X$, and (ii) $s^{\prime \prime}(z)=s^{\prime}(z)$ for each non-clock $z \in Z$. As $s \cup\left\{\delta \mapsto \delta_{s}\right\} \cup\left\{y^{\prime} \mapsto s^{\prime}(y) \mid y \in X \cup Z\right\} \models \mathcal{T}$, we have $\delta_{s} \geq 0$ and for each $x \in X$ either $s^{\prime}(x)=0$ or $s^{\prime}(x)=s(x)+\delta_{s}=s^{\prime \prime}(x)$. That is, intuitively $s^{\prime \prime}$ is obtained from $s^{\prime}$ by "unresetting" the reset clocks.

Next, take any $\delta_{t} \in \mathbb{R}$ and state $t^{\prime \prime}$ such that (i) $\delta_{t} \geq 0$, (ii) $\delta_{t}=0 \Leftrightarrow \delta_{s}=0$, (iii) $t^{\prime \prime}(x)=t(x)+\delta_{t}$ for each clock $x \in X$, (iv) $t^{\prime \prime}(z)=s^{\prime}(z)$ for each non-clock $z \in Z$, and (v) $s^{\prime \prime} \approx t^{\prime \prime}$. Such $\delta_{t}$ and $t^{\prime \prime}$ exists because $s \approx t$ and of the fact that clock valuations in the same region have time successors in same regions [1]. Let $t^{\prime}$ be the state such that (i) for each $x \in X, t^{\prime}(x)=0$ if $s^{\prime}(x)=0$ and $t^{\prime}(x)=t^{\prime \prime}(x)=t(x)+\delta_{t}$ otherwise, and (ii) $t^{\prime}(z)=t^{\prime \prime}(z)=s^{\prime}(z)$ for each non-clock $z \in Z$. Now $s^{\prime} \approx t^{\prime}$. As a summary, intuitively $t^{\prime}$ is a state in the region that is obtained by letting time pass in the similar manner as when moving from $s$ to $s^{\prime}$ and then resetting the same clocks.

Now we only have to show that $t \cup\left\{\delta \mapsto \delta_{t}\right\} \cup\left\{y^{\prime} \mapsto t^{\prime}(y) \mid y \in X \cup Z\right\} \models \mathcal{T}$. We do this by showing that the atoms in $\mathcal{T}$ evaluate to the same boolean value under both $s \cup\left\{\delta \mapsto \delta_{s}\right\} \cup\left\{y^{\prime} \mapsto s^{\prime}(y) \mid y \in X \cup Z\right\}$ and $t \cup\left\{\delta \mapsto \delta_{t}\right\} \cup\left\{y^{\prime} \mapsto t^{\prime}(y) \mid y \in X \cup Z\right\}$.

- Case: the atom does not involve variables in $X \cup X^{\prime} \cup$ $\{\delta\}$.

In this case the atom evaluates to true under $s \cup$ $\left\{y^{\prime} \mapsto s^{\prime}(y) \mid y \in X \cup Z\right\}$ if and only if it does under $t \cup\left\{y^{\prime} \mapsto t^{\prime}(y) \mid y \in X \cup Z\right\}$ because $s \approx t$ and $s^{\prime} \approx t^{\prime}$. 
- Case: the atom is of form $x^{\prime}=0$.

Because $s^{\prime} \approx t^{\prime}$, the atom evaluates to true under $\left\{y^{\prime} \mapsto s^{\prime}(y) \mid y \in X \cup Z\right\}$ if and only if it does under $\left\{y^{\prime} \mapsto t^{\prime}(y) \mid y \in X \cup Z\right\}$

- Case: the atom is of form $x^{\prime}=x+\delta$.

We have to consider the following:

1) Sub-case $s^{\prime}(x)=0$

Thus $t^{\prime}(x)=0$ as well because $s^{\prime} \approx t^{\prime}$. Now $x^{\prime}=x+\delta$ evaluates to true under $s \cup\left\{\delta \mapsto \delta_{s}\right\} \cup$ $\left\{y^{\prime} \mapsto s^{\prime}(y) \mid y \in X \cup Z\right\}$ if and only if $s(x)=0$ and $\delta_{s}=0$ (as $x$ and $\delta$ always have non-negative values).

a) If $s(x)=0$ and $\delta_{s}=0$, then $t(x)=0$ and $\delta_{t}=0$ as well because $s \approx t$ and $s^{\prime \prime} \approx t^{\prime \prime}$ (forcing that $s(x)+\delta_{s}=0$ if and only if $t(x)+$ $\left.\delta_{t}=0\right)$.

b) If $s(x)>0$, then $t(x)>0$ as $s \approx t$, and thus $s^{\prime}(x) \neq s(x)+\delta_{s}$ and $t^{\prime}(x) \neq t(x)+\delta_{t}$.

c) If $s(x)=0$ and $\delta_{s}>0$, then $t(x)=0$ as $s \approx t$ and $\delta_{t}>0$ as $s^{\prime \prime} \approx t^{\prime \prime}$ and $s^{\prime \prime}(x)=$ $s(x)+\delta_{s} \geq 0$, implying that $s^{\prime}(x) \neq s(x)+\delta_{s}$ and $t^{\prime}(x) \neq t(x)+\delta_{t}$.

2) Sub-case $s^{\prime}(x)>0$.

Now also $t^{\prime}(x)>0$ as $s^{\prime} \approx t^{\prime}$. As $s^{\prime}(x)>0$, it must be that $s^{\prime}(x)=s(x)+\delta_{s}$ of the restriction imposed on $\mathcal{T}$. By the construction of $t^{\prime \prime}$ and $t^{\prime}$, $t^{\prime}(x)=t^{\prime \prime}(x)=t(x)+\delta_{t}$.

- Case: the atom is of form $x \bowtie n$.

Because $s \approx t$, the atom evaluates to true under $s$ if and only if it does under $t$.

- Case: the atom is of form $x+\delta \bowtie n$.

By the construction of $s^{\prime \prime}$ and $t^{\prime \prime}$, and the fact that $s^{\prime \prime} \approx$ $t^{\prime \prime}$, we have that $s^{\prime \prime}(x)=s(x)+\delta_{s} \bowtie n$ if and only if $t^{\prime \prime}(x)=t(x)+\delta_{t} \bowtie n$.

- Case: the atom is of form $\delta \bowtie 0$.

Because $\delta_{s} \geq 0, \delta_{t} \geq 0$, and $\delta_{s}=0 \Leftrightarrow \delta_{t}=0$, the atom evaluates to true under $\left\{\delta \mapsto \delta_{s}\right\}$ if and only if it does under $\left\{\delta \mapsto \delta_{t}\right\}$. 